\title{
Environmental Impact Assessment of Agricultural Production Using LCA: A Review
}

\author{
Rahmah Alhashim ${ }^{1}$, Raveendranpillai Deepa ${ }^{2}$ (D) and Aavudai Anandhi ${ }^{3, *(D)}$ \\ 1 School of the Environment, Florida Agricultural and Mechanical University, Tallahassee, FL 32307, USA; \\ rahmah1.alhashim@famu.edu \\ 2 College of Agriculture and Food Sciences, Florida Agricultural and Mechanical University, \\ Tallahassee, FL 32307, USA; deepa1.raveendranpil@famu.edu \\ 3 Biological Systems Engineering, Florida Agricultural and Mechanical University, Tallahassee, FL 32307, USA \\ * Correspondence: anandhi@famu.edu; Tel.: +1-850-412-5000
}

Citation: Alhashim, R.; Deepa, R.; Anandhi, A. Environmental Impact Assessment of Agricultural Production Using LCA: A Review. Climate 2021, 9, 164. https://doi.org/ $10.3390 /$ cli9110164

Academic Editor: Steven McNulty

Received: 30 August 2021

Accepted: 29 October 2021

Published: 5 November 2021

Publisher's Note: MDPI stays neutral with regard to jurisdictional claims in published maps and institutional affiliations.

Copyright: (c) 2021 by the authors. Licensee MDPI, Basel, Switzerland. This article is an open access article distributed under the terms and conditions of the Creative Commons Attribution (CC BY) license (https:// creativecommons.org/licenses/by/ $4.0 /)$.

\begin{abstract}
Life cycle impact assessment (LCA) provides a better understanding of the energy, water, and material input and evaluates any production system's output impacts. LCA has been carried out on various crops and products across the world. Some countries, however, have none or only a few studies. Here, we present the results of a literature review, following the PRISMA protocol, of what has been done in LCA to help stakeholders in these regions to understand the environmental impact at different stages of a product. The published literature was examined using the Google Scholar database to synthesize LCA research on agricultural activities, and 74 studies were analyzed. The evaluated papers are extensively studied in order to comprehend the various impact categories involved in LCA. The study reveals that tomatoes and wheat were the major crops considered in LCA. The major environmental impacts, namely, human toxicity potential and terrestrial ecotoxicity potential, were the major focus. Furthermore, the most used impact methods were CML, ISO, and IPCC. It was also found that studies were most often conducted in the European sector since most models and databases are suited for European agri-food products. The literature review did not focus on a specific region or a crop. Consequently, many studies appeared while searching using the keywords. Notwithstanding such limitations, this review provides a valuable reference point for those practicing LCA.
\end{abstract}

Keywords: meta-analysis; GHG emission; ecotoxicity; agriculture; crop production; LCA

\section{Introduction}

Food supply chains (FSCs) are very complex. There are many components involved in FSCs that process, produce, package, store, transfer, distribute, and market food products to final consumers [1]. Each element in the FSC process is essential, as in any other supply chain; a change in one component affects the others. The relationship between the food system and the economy, environment, and society is mentioned by some organizations and agencies, such as the Food and Agricultural Organization (FAO), Institute of Medicine (IOM), and National Research Council (NRC), when they define the FSC [2]. Therefore, the most crucial question is as follows: Which food production system is more sustainable for the environment and communities?

There are many concerns about food resources and massive population growth, such as meeting the food demand for the world's population, production, and food consumption [1]. The total crop production must double or increase by at least $70 \%$ to meet the increasing world population's demand by 2050 [3]. Models have estimated that a $2.4 \%$ annual increase in crop yield is necessary to reach the 2050 demand [4]. The rise in food demand results in substantial energy and resource use by the food supply chain, leading to different environmental impacts. Many organizations have mentioned environmental impacts associated with food production, including the use of land, water, and climate 
change. Significant environmental challenges that humans face are primarily due to climate change and the predicted future shortage of fossil fuels [5]. Farming methods, fertilizers, pesticides, water pumping, tractors to prepare the land, and transport of the crops or final food products via railroads, trucks, airplanes, or ships can all impact the environment. Lastly, food processing and food preservation methods such as refrigeration and packaging also contribute to environmental damages. There are many production sectors involved in environmental impacts, and one of them is the agricultural sector.

According to the Environmental Protection Agency (EPA), agricultural chemicals and pesticide manufacturing are two of the 68 area source groups that account for $90 \%$ of the overall emissions of the 30 urban air toxins. For example, in 2018, greenhouse gas (GHG) emissions from the agriculture economic sector accounted for $9.9 \%$ of total US greenhouse gas emissions. Furthermore, GHG from agriculture has increased by $10.1 \%$ since 1990 [6]. One of the direct greenhouse gases is nitrous oxide. Agricultural soil management operations such as synthetic and organic fertilizers and other cropping techniques, the management of manure, and the burning of agricultural wastes produce nitrous oxide. Agricultural soil management is the major source of $\mathrm{N}_{2} \mathrm{O}$ emissions in the US, accounting for around 75\% of total emissions [7]. Agricultural soils, for example, are a major source of $\mathrm{NO}_{\mathrm{x}}$ pollution in California, with soil $\mathrm{NO}_{\mathrm{x}}$ emissions in the state's Central Valley region being particularly high. Therefore, it is necessary to quantify the impacts of agricultural products along the food supply chain for sustainable production and consumption systems.

Since the number of operations in the food system is large and complex, many studies have used the life cycle assessment (LCA) methodology as a tool to study the overall resources used and the environmental impact of food products over its entire life cycle [8]. It is best known for its qualitative and quantitative analysis of a product's environmental aspects over its whole life cycle [9]. Products in this context include both goods and services [10]. Environmental impacts in the LCA context refer to the adverse effects on the areas of concern such as the ecosystem, human health, and natural resources. Due to the limitation of raw materials and energy resources, LCA has been used since the 1960s to find solutions for sustainable productions [11].

Such research on the crop supply chain provides helpful information from the economic, social, and environmental perspectives. Using the LCA offers a better understanding of the energy, water, and material input and evaluates the outputs' impacts. Thus, decisionmakers in various fields can regulate new policies and use modern practices to improve the production supply chains. As observed in previous studies $[9,12]$, many authors have used LCA to address environmental impacts over the entire life cycle of crops. However, the world's largest industrial sector, the food supply chain, involves various crops and products that still need to be addressed by the LCA.

Therefore, this study's broad objective is to synthesize the LCA studies relating to different environmental impacts from agricultural production to support stakeholders with decision-making. Besides, an in-depth analysis of the various steps involved in LCA is provided.

\section{Materials and Methods}

A literature review of published articles in international journals was undertaken using the Preferred Reporting Items for Systematic Reviews and Meta-Analyses (PRISMA) protocol to address the research aims.

\subsection{Eligibility Criteria}

The studies that applied the following selection criteria were chosen to reduce the number of articles: (i) using the LCA method, (ii) including GHG in their impact category and/or ecotoxicity, and (iii) researching agriculture products. A total of 36 research articles were eliminated because they were about FSCs and not GHG/ecotoxicity as an effect cate- 
gory, did not apply the LCA methodology, or utilized the LCA method for nonagricultural products. The LCA studies were analyzed extensively considering four phases of the LCA:

- Goal and scope definition,

- Life cycle inventory,

- Life cycle impact assessment,

- Life cycle interpretation/recommendation options.

\subsection{Search Strategy}

The literature review was done through the Google Scholar database. The keyword "LCA crop production" was used in the initial step, which yielded 59,100 studies as of July 2021. Later, more specific keywords were used, such as "agri-food supply chain and LCA" and "agri-food supply chain and GHG" combined with different fruit and vegetable products such as corn, peanuts, wheat, tomato, and apple. Nevertheless, the number of studies available remained enormous, the largest number of articles we got when we used the above key word with different crops was 7330, while the smallest number was 1820 . A total of 110 articles were downloaded and analyzed. Twenty-nine studies were excluded because they were about FSCs and not about GHG/ecotoxicity as an effect category, or because they utilized the LCA method.

Furthermore, seven more were excluded because they used the LCA method for non-agricultural products. Accordingly, we ended up with 74 articles after applying the selection criteria. Figure 1 shows the steps used throughout the review and the inclusion criteria for the literature.

\section{Identification of studies via databases}

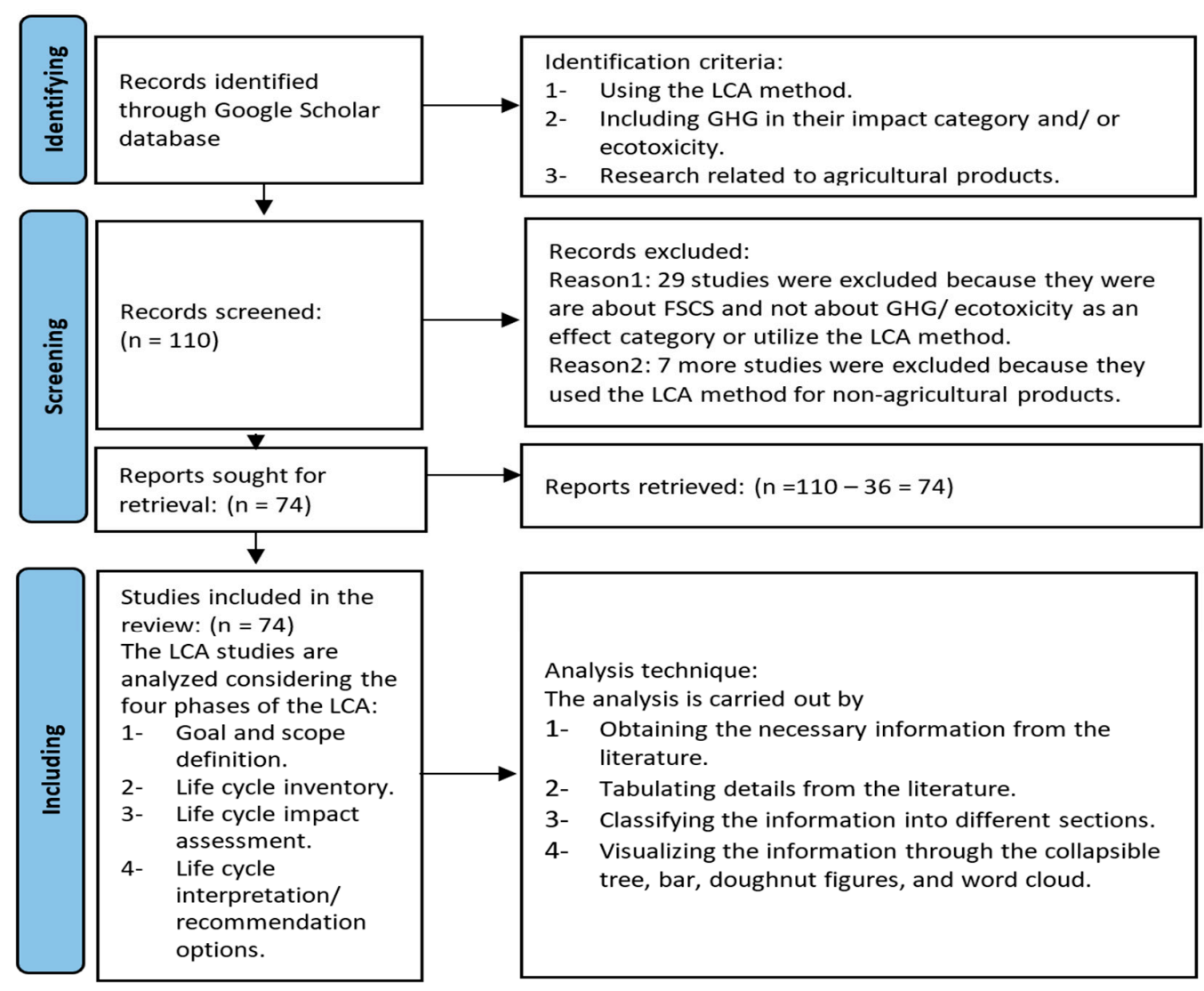

Figure 1. Steps followed for review and the inclusion/exclusion criteria. 


\subsection{Categorization}

The data obtained from the reviewed articles included the year of study, the aim of the study, and the different steps involved in LCA assessment, which are discussed in the results section. The timeline, different components, the approach of the LCA, application of the LCA concept in the impact analysis, and suggestions for a sustainable food system are all covered.

\subsection{Data Analysis}

The analysis was carried out by obtaining the necessary information from the literature, as given in Tables A1 and A2 (Appendix A). Then, the information was visualized by means of collapsible trees, bar charts, doughnut figures, and word clouds after the information was classified into different result sections. Word clouds have evolved as a straightforward and visually appealing technique of text representation. They are used in a variety of contexts to offer an overview by reducing text down to the most frequently occurring terms. This is usually done statistically as a pure text summary $[13,14]$. Word clouds can be the initial step to refine the important concepts of results, which could save a great deal of time for other researchers since they already know where to start and the most common terms and ideas [15]. Pie and doughnut charts represent the relationship of parts with the whole $[16,17]$. Collapsible trees, bar charts, and doughnut figures are designed to provide greater numerical detail. Combining word clouds and bar charts allowed presenting both qualitative and quantitative information on LCA results.

The collapsible tree diagram was created with R software Version 3.6.1, and the bar and doughnut figures were created with Microsoft Excel. When making word cloud figures using the word cloud online website (https:/ / www.jasondavies.com/wordcloud/ accessed on November 2021), each word must be typed correctly since the size and the color of the words in the figure are affected by the number of words entered. Therefore, it is essential to make sure that the number of entered words is accurate.

Lastly, the study was organized in IMRAD format, which is the most common format for scientific papers. The term represents the first letters of the words introduction, materials and methods, results, and discussion. IMRAD format facilitates knowledge acquisition and enables easy evaluation of an article [18]. Currently, IMRAD is used by the majority of academic publications. Before the IMRAD structure, all academic writing followed the IBC (introduction, body, and conclusion) pattern. The IMRAD format is only a more specified variant of the IBC format. [19]. It is important to keep in mind that no one journal follows a standard or consistent format. Each journal has its structure, yet they all have a guideline for authors [20].

\section{Results}

\subsection{Snapshot of Selected Studies}

The characteristics of publications during 1998-2021 are displayed in Figure 2 to obtain an overview of LCA research. The number of publications per year has increased steadily since 2008, following development of the ISO standard.

Critiques of the ISO 14040 series pre-2006 were that LCA is too nascent [21], and ISO 14040 does not address uncertainty, weighting, valuation, and allocation [22].

The release of the latest version of the ISO 14040 standard in 2006 explains why LCA research is attracting more attention. Moreover, some have recently gone so far as to state that the ISO 14040: 2006 series "has proven a suitable tool for sustainability assessment" [13,14]. Fava et al. (2009) claimed that ISO 14040 should be the basis for future LCA studies [23]. 


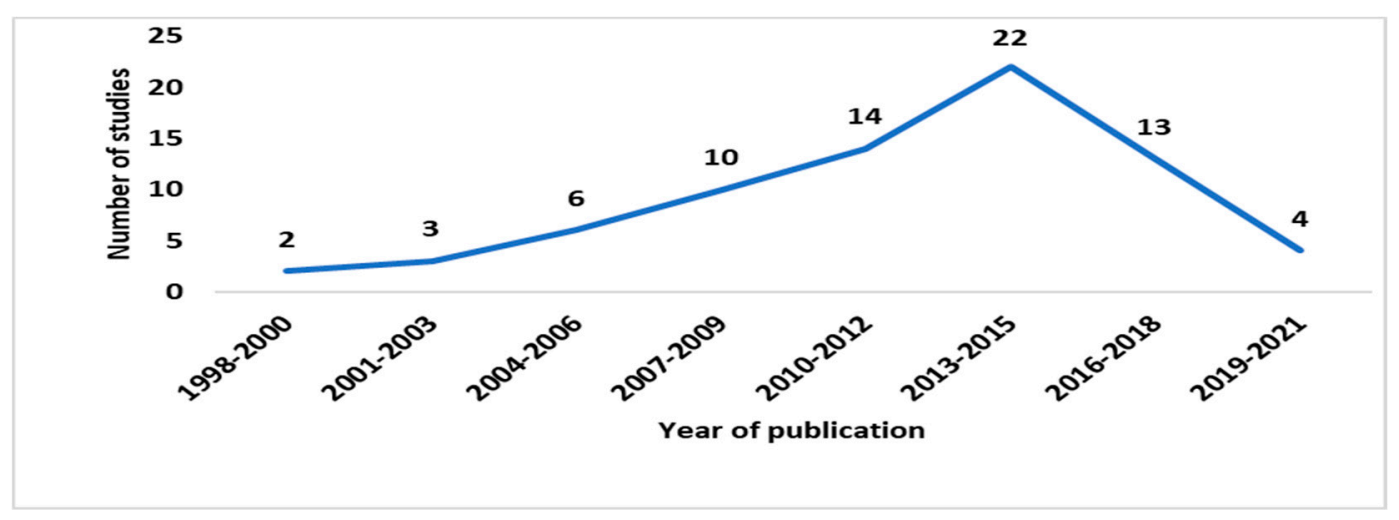

Figure 2. Frequency of studies related to LCA of agricultural production from 1998 to 2021 ( $n=74$ ).

Studies found that the most common tool to study the impact on the environment associated with a product over its life cycle in the agri-food sector was the LCA ISO 14040 standard [14,15]. LCA ISO 14040 has four main phases: (1) goal and scope, which is the essential component of the LCA, (2) qualitative and/or quantitative inventory analysis of the used resources and the emissions released from the life cycle of a product, (3) life cycle impact assessment, which can be divided into classification, characterization, and evaluation, and (4) the interpretation, involving the identification of key issues, evaluation (including checking completeness, sensitivity, and consistency), and development of conclusions together with recommendations, as defined by ISO 14043 (Figure 3). The details of each phase are discussed below.

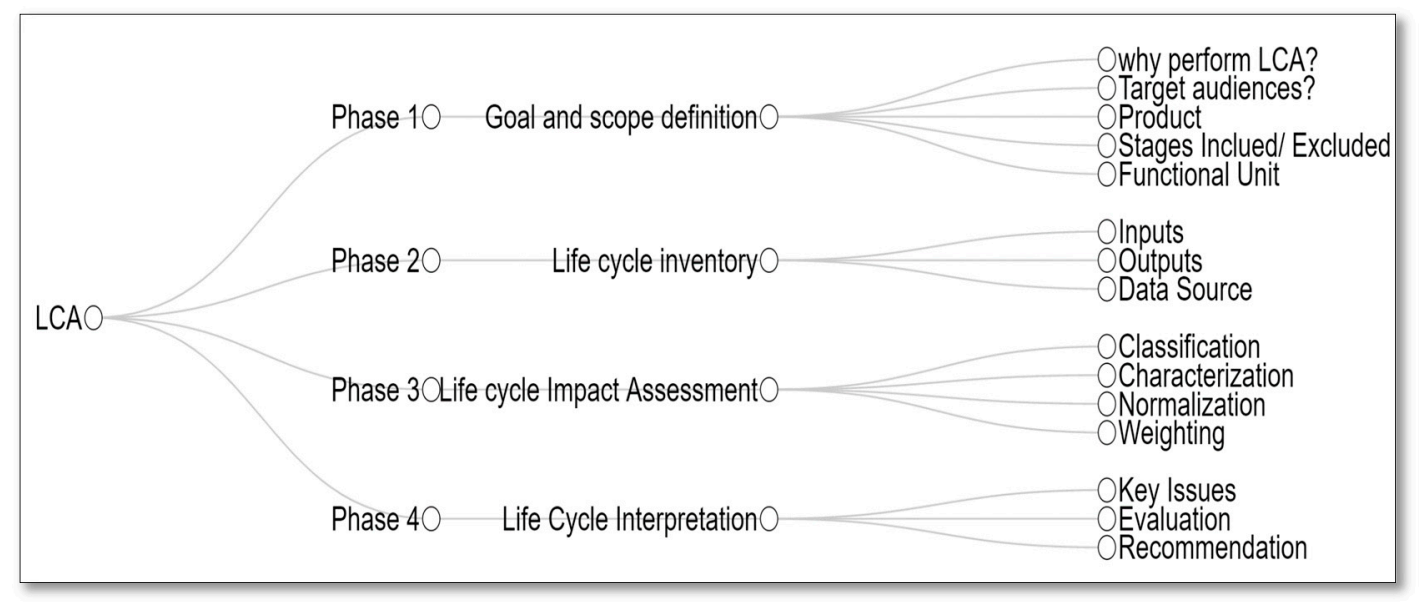

Figure 3. Overview of life cycle assessment (LCA) phases.

\subsection{Phase 1: Goal and Scope Definition}

\subsubsection{Goal}

According to Lee and Inaba (2004), the following questions should be addressed to set up the goal: Why perform LCA, who is the target audience, and what is the product under the LCA study [10]? These were recognized from the reviewed articles while examining the first phase of the LCA, as given in Figure 4. Some of the studies stated the answers to these questions directly, whereas others addressed them indirectly. Figures 5-7 show the most common responses to each question. 


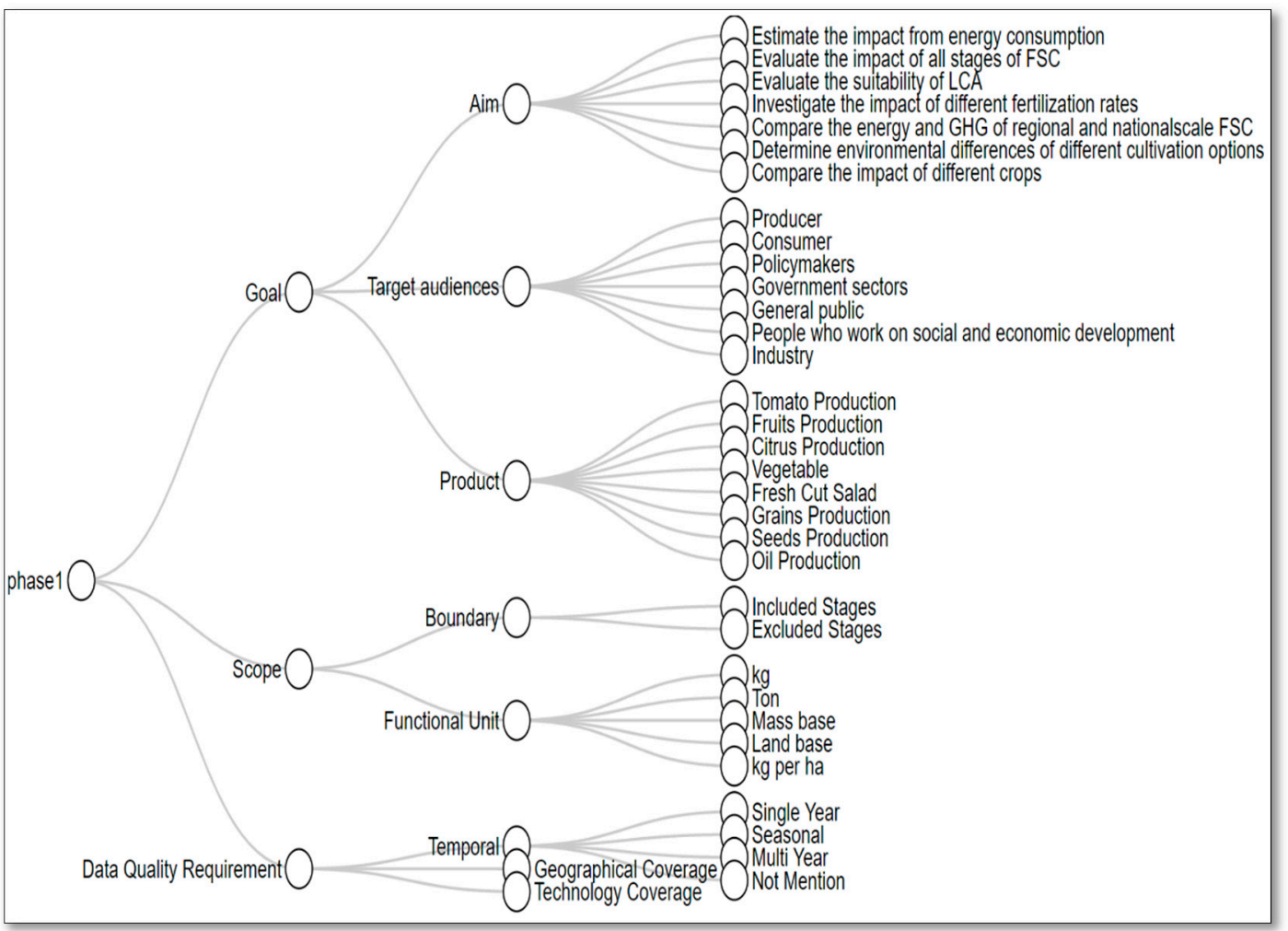

Figure 4. Phase 1 (goal and scope definition) of life cycle assessment (LCA).

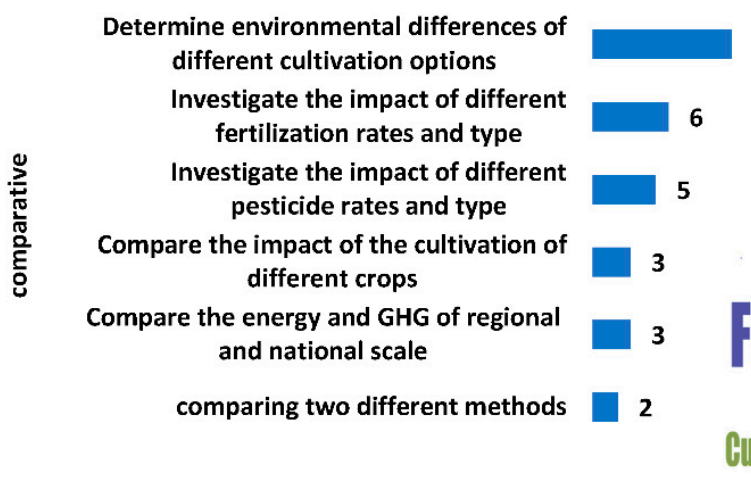

11

options

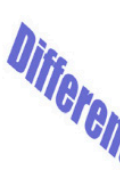

Evaluate the impact of all or most stages of

$$
\text { FSC }
$$
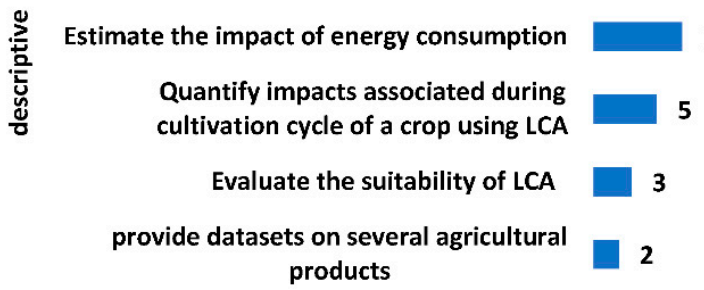
$\begin{array}{llllllll}0 & 5 & 10 & 15 & 20 & 25 & 30 & 35\end{array}$
Number of Study

Figure 5. Quantitative and qualitative representation of the common aims of LCA from the literature. 


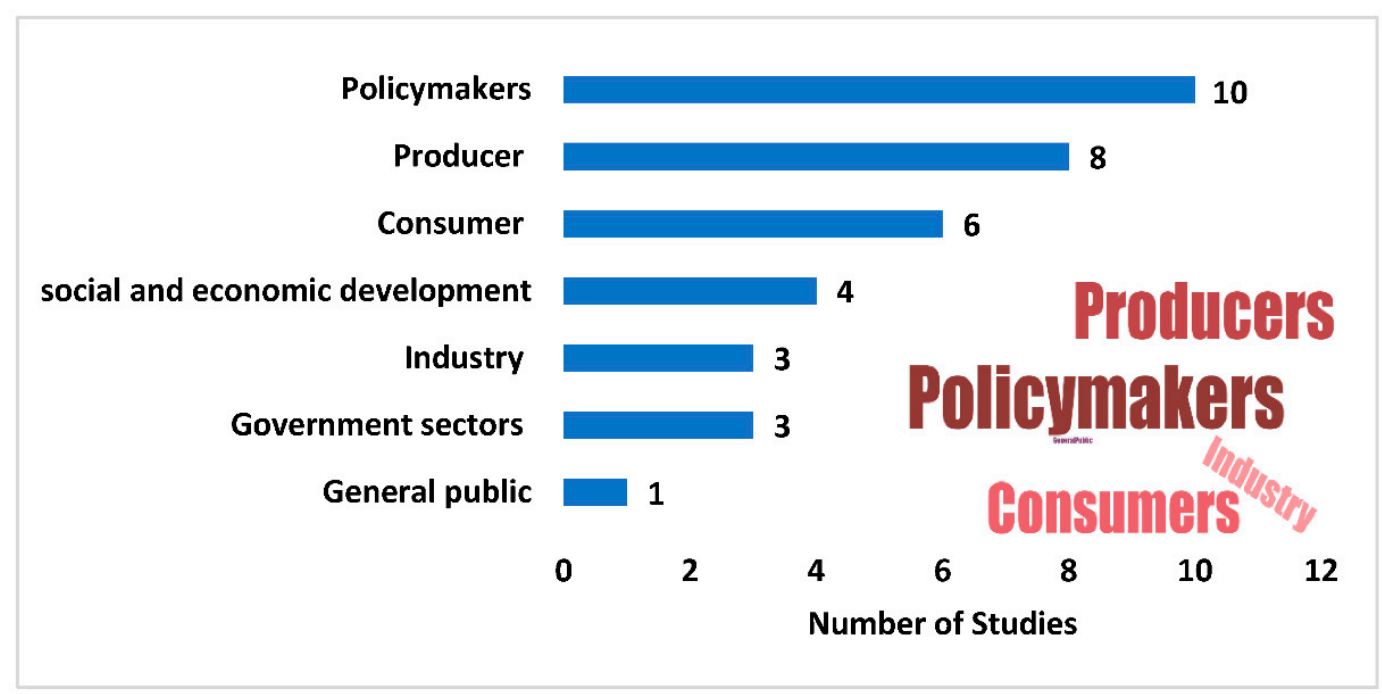

Figure 6. Target audiences in the literature represented as bars (quantitative) and a word cloud (qualitative).

\section{Aims of LCA}

As indicated in the literature, LCA studies can be partitioned into two major categories: descriptive and comparative. Descriptions aim to recognize the natural load of a chosen framework, while comparisons aim to differentiate between two frameworks. Among the discussed papers, 48 were descriptive, while 30 were comparative. As noted, the most common aim was to assess agricultural production, cultivation, processing, packaging, transport, and emission at all production stages to recognize the vast issues and to propose reasonable alternatives that decrease the environmental effects (Figure 5). The purpose of this review was to better understand how to use LCA to evaluate the environmental impact of agricultural production. The least common goal was to compare LCA to other methods, which may be due to the difficulty of making a fair comparison in terms of method performance.

\section{Target Audience}

The target audience defines who undertakes or commissions an LCA and for whom. It is critical to understand who will use the LCA results to provide them with helpful information. The majority of articles have multiple target audiences (TAs). Politicians working on climate change, decision-makers, and policymakers on global warming potential (GWP) footprints related to food and common agricultural policy (CAP) were the most common TAs, with 10 studies. Additionally, several studies targeted government sectors such as food sector policymakers, the country's agriculture sector, and the fruit and vegetable sector. Following that, the producers, namely, the farmers and the producing industry, were targeted in eight studies, six of which provided information to the consumer on a local and international scale (see Figure 6). People working on social and economic development, such as government policymakers for sustainable consumption and production, future ecolabeling programs, and those working to improve the environmental and financial sustainability of existing agricultural systems, were also targeted. Another target audience was represented by the Florida food, agri-food, and citrus industries. As shown in Figure 6, only 35 of the 74 research articles analyzed clearly stated their target audience. The frequency of target audiences is also displayed as a word cloud for a rapid overview. 


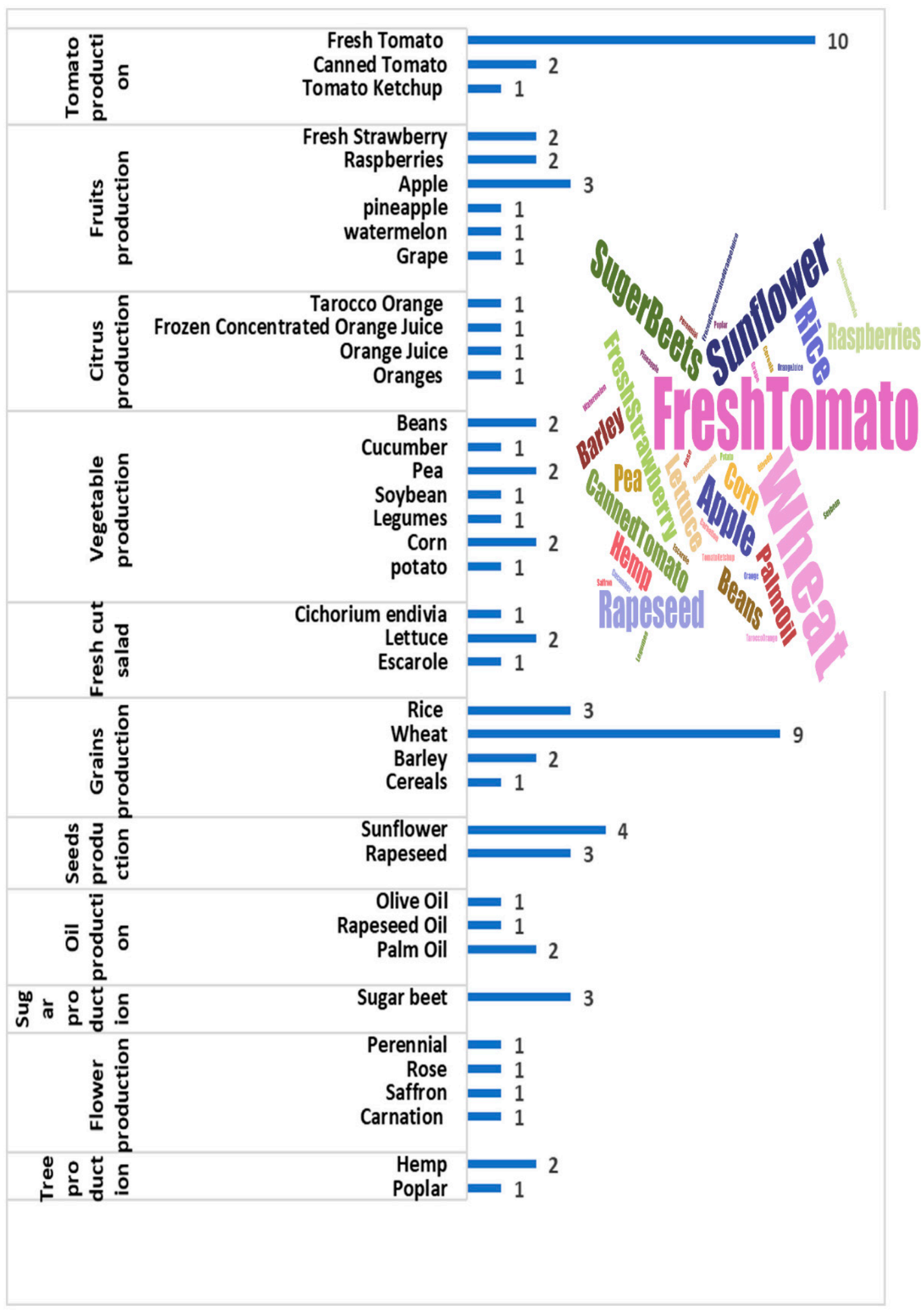

Figure 7. Common agricultural products used in LCA studies. 


\section{Agricultural}

We divided the products into 11 categories: tomato, fruits, citrus, vegetable, fresh salad, grains, seeds, oil, sugar, flower, and trees, as shown in Figure 7. The most common product was tomato; 13 studies analyzed tomato production, including fresh tomato, canned tomato (whole peeled, paste, and diced), and ketchup. The second most common product was wheat with nine studies. Because some studies involved more than one crop, that explains why the same reference was used for multiple crop groups and why the number of studies on the chart exceeds the number of studies covered. Tomato production was separated into three categories since three types of tomato products (fresh tomato, canned tomato, and tomato ketchup) were considered, as indicated in the diagram.

\subsubsection{Scope}

The scope defines the product system boundaries that determine which unit processes should be included in the LCA analysis and which should be excluded. Table A2 (Appendix A) includes more information on all 74 studies, including their inputs and outputs inside and outside of the scope. Most studies (14) contained three to four phases in their boundaries, as shown in Figure 8A. There are two explanations for not including the eliminated phases in the majority of articles. The first is a lack of data and knowledge about individual inputs, making it difficult to get a decent overall view. Secondly, some authors excluded the minor influence stages because it was impossible to include all phases.

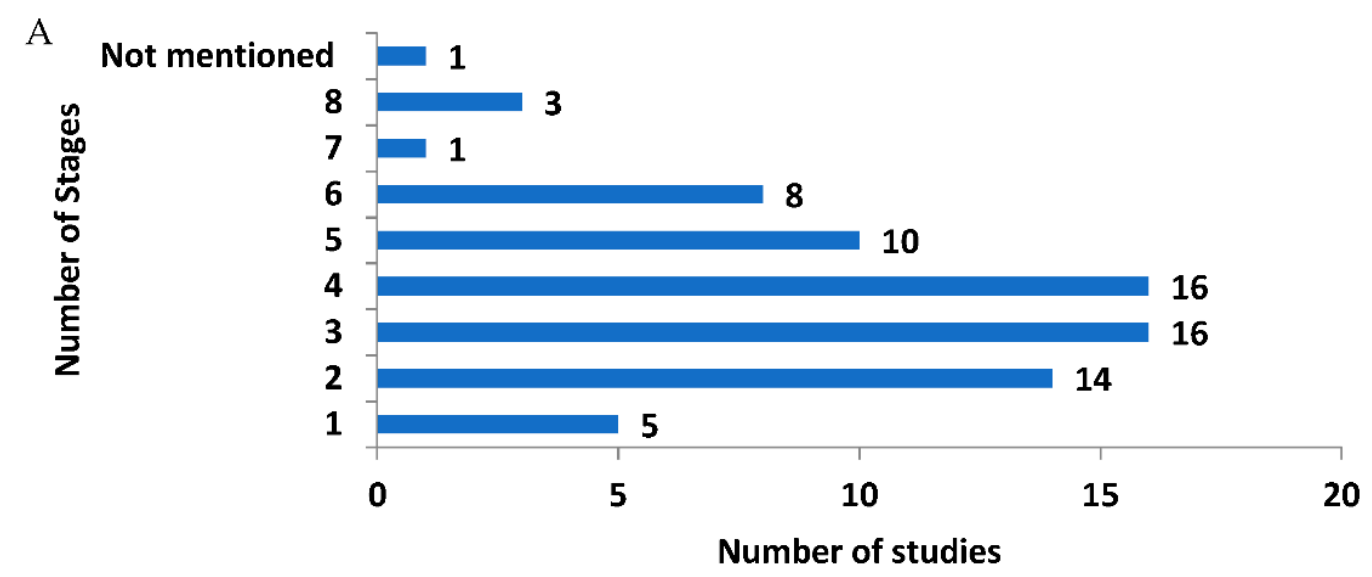

B

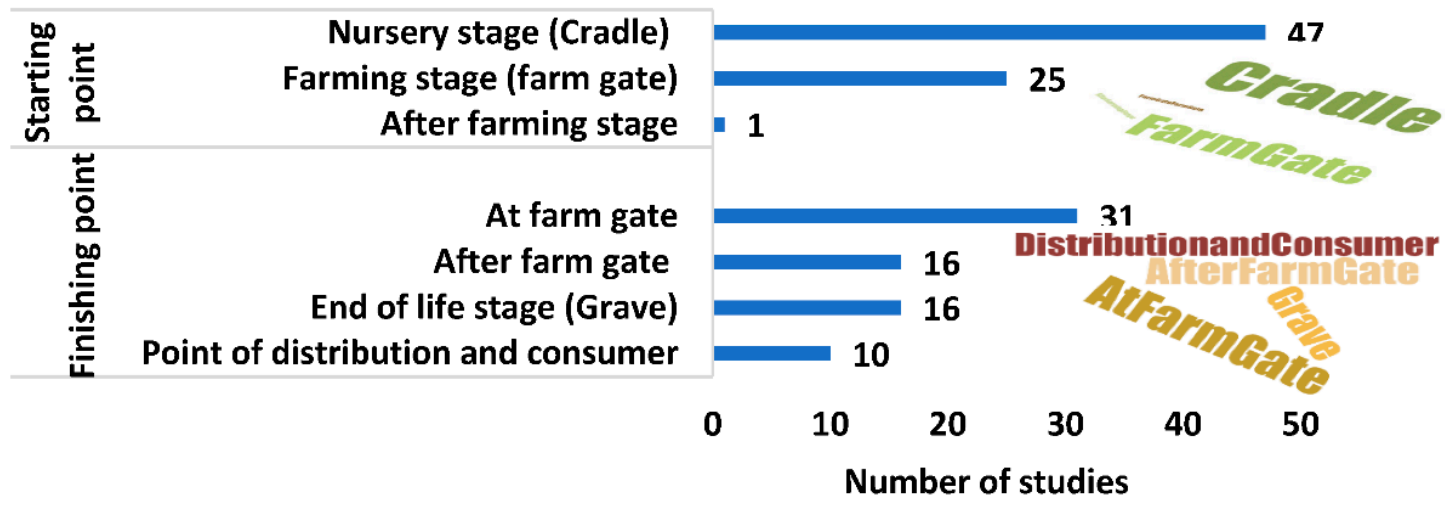

Figure 8. (A) Number of stages included in the systems obtained from the literature; (B) quantitative and qualitative illustration of the starting and ending points of the included stages as boundaries.

Since we are looking at the agri-food supply chain, most of the articles noticeably had similar steps when designing their boundaries. Depending on the selected crop and the target audience, there were slight differences in the scope's starting point and 
finishing point (Figure 8B). According to the review, 47 studies started their scope from the nursery stage (cradle), which involves preparing the raw materials, buildings, and field or land. Furthermore, 25 studies began their scope from the farming stage (farm gate). Considering our focus on agricultural production, only one study started their scope after the farming stage.

Similarly, the final stage differed from one study to another, ranging from the farming stage to the grave, including the product's processing, packaging, storing, and transferring stages. Thirty-one studies in the literature review included steps until the crop harvesting stage, whereas 16 authors included some or all of the processing, packaging, and storing stages in the study's scope. A number of reviewed studies reached the point of distribution and consumption in their analysis. Disposal and waste management were the final stages in some studies, with 10 articles including the end-of-life phase in their analysis (Figure 8B). One study did not specific boundaries; thus, the number in Figure 8B is less than the number of studies reviewed [24].

\subsubsection{Functional Unit}

Another step of the goal and scope phase is to choose a functional unit of the scope. A functional unit is the reference unit in which elementary flows from the inventory until the impact assessment stage are represented. Selecting the ideal functional unit is necessary during the boundary designation step. The functional unit is dependent on the type of input materials (raw material) and the final products. Accordingly, the input unit might be separate from the outputs. For example, the output such as GHG emissions could be in $\mathrm{kg} \cdot \mathrm{ha}^{-1}$ while the final product could in tons or the input material could be in $\mathrm{kWh}$ for energy consumption and $\mathrm{kg}$ for fertilizers. Figure 9 shows the most common functional units used in previous studies.

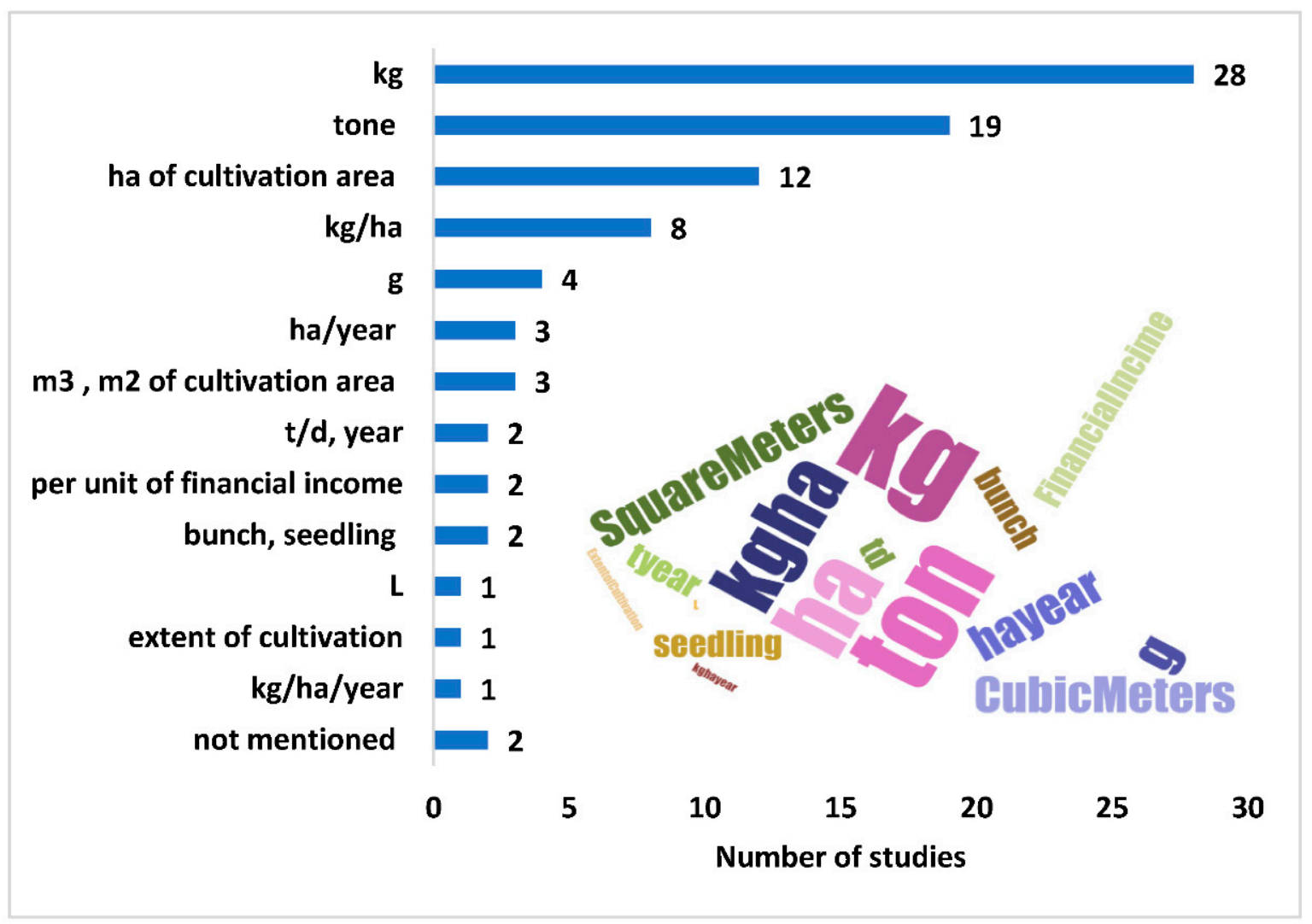

Figure 9. Functional units identified from the studies. 


\subsubsection{Data Quality Requirement}

The reliability of the results from LCA studies strongly depends on how data quality requirements are met. The following parameters should be considered: time-related coverage (selected year), geographical coverage (study area), and technology coverage (technology used in the processes stages). This paper examined the temporal and spatial data in detail and the used machinery in general.

It is understood from the literature review that most studies collected their data for a single year of cultivation (Figure 10B). The spatial scale of the analysis (global or regional) depends on the impact category. For example, global warming is a worldwide issue, whereas acidification is a regional issue. Furthermore, two countries were commonly represented in the evaluated research, Italy and the United States, with 17 and 14 studies, respectively (Figure 10A). When it comes to the technology used in each activity, the majority of the tools mentioned were agricultural equipment, which is to be expected given that we are investigating crop production.

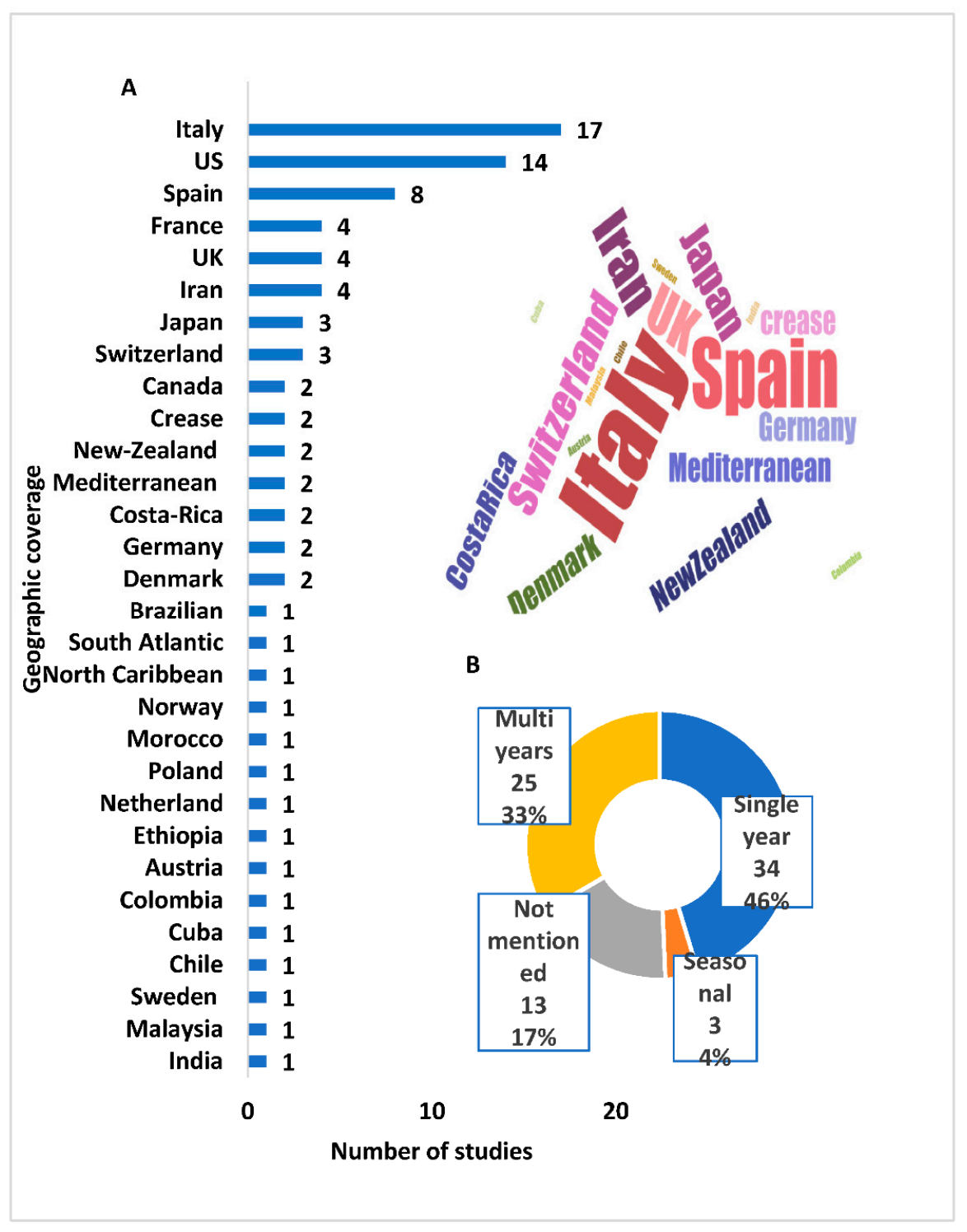

Figure 10. (A) Quantitative (bars) and qualitative (word cloud) representation of the geographic coverage considered in the reviewed studies; (B) donut chart depicting the temporal scales used in the literature. 


\subsection{Phase 2: Life Cycle Inventory}

The second step of the LCA is the life cycle inventory analysis (LCI). The product's life cycle inventory results in an LCA study are obtained by summing up all fractional contributions of the input and output from each unit process in the product's production system. Thus, LCI generates quantitative environmental information of a product throughout its entire life cycle.

Most studies at this stage specified the input material (water, fertilizer, pesticide, diesel, etc.) in each process of the production included in the scope, as well as the output (harvested crop, waste, emission to the air, soil, and water, etc.). Furthermore, they mentioned the sources of the inventory data (Figure 11), typically being from primary and/or secondary data sources. Primary data are obtained from specific processes throughout the life cycle of the researched product. Process activity data (physical measures of a process that results in GHG emissions or removal), direct emissions data (determined through direct monitoring, stoichiometry, mass balance, or similar methods) from a specific site, or data averaged across all sites containing the specific process are all examples of primary data [25]. Secondary data are collected from government departments, organizational records, and studies that previously gathered information from primary sources and made it available to other researchers.

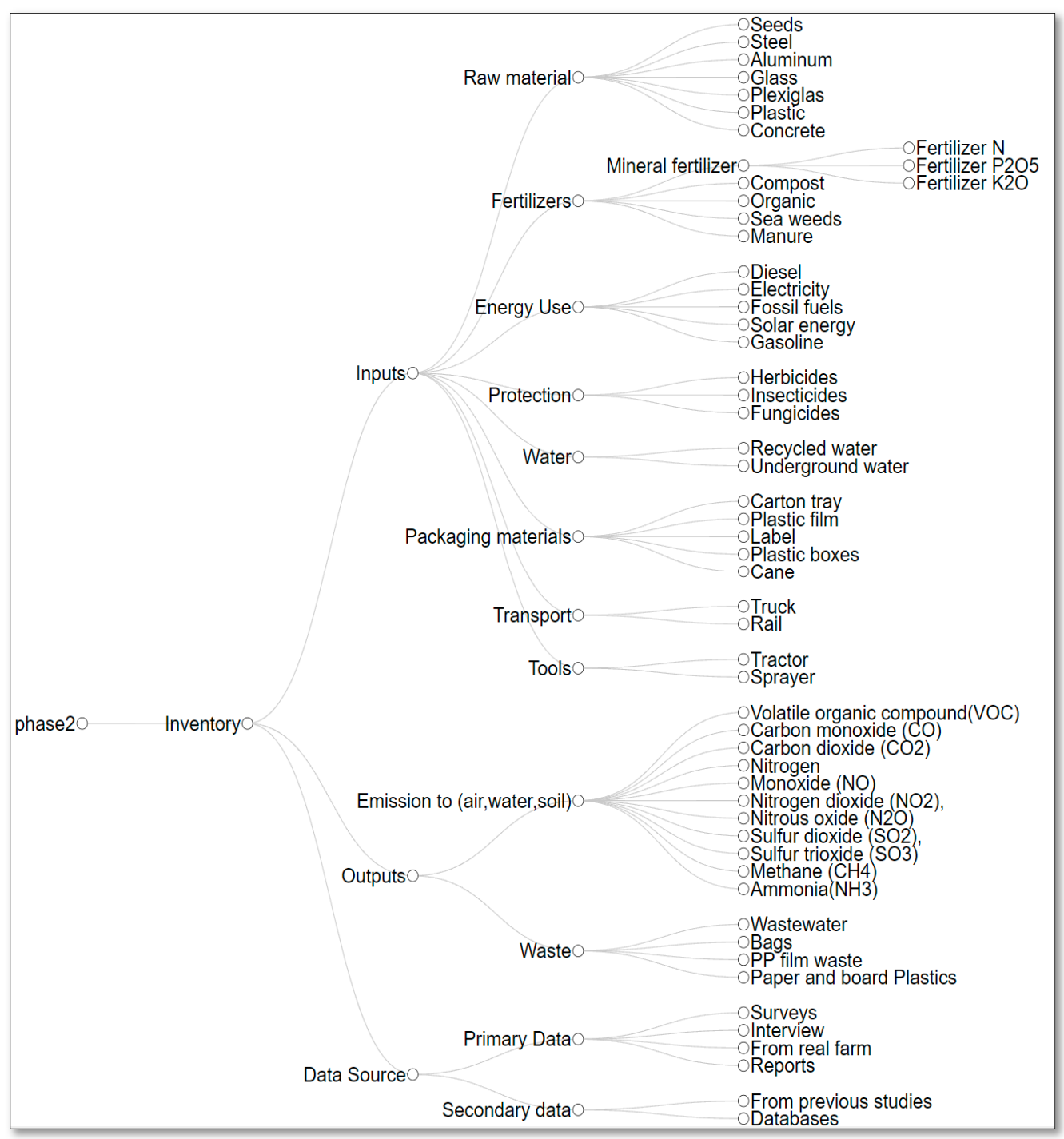

Figure 11. Phase 2 (inventory) of life cycle assessment (LCA). 
About $48 \%$ of the studies used secondary data, $13 \%$ used primary data, and $35 \%$ used both. One study collected data from a real farm experience. Three authors conducted interviews with owners to collect the data. Two studies used surveys with specific questions to collect the required information. One study mentioned that the source was primary, but the article did not specify their method. Seven studies utilized primary data, while the other nine used secondary data. The authors of the examined research utilized two types of secondary data methods: databases and previous studies. Eleven of the studies used databases, while five of them used previous studies. Five writers, on the other hand, gathered inventory data from databases and prior studies. Twenty-six studies utilized both primary and secondary approaches to reduce the uncertainty of their findings (Figure 12A,B).
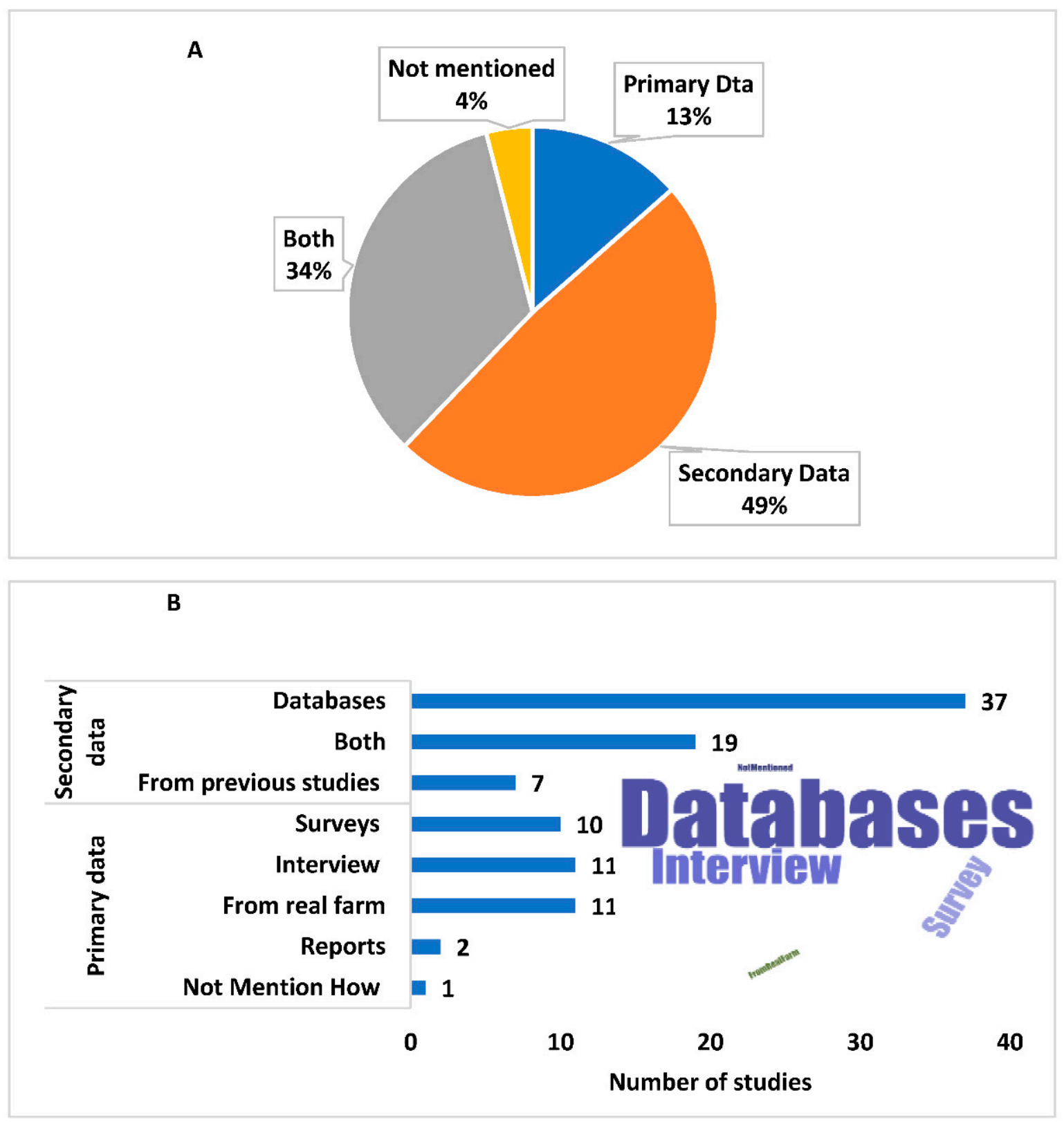

Figure 12. (A) Data sources of the inventory stage rendered as a pie chart; (B) breakdown of primary and secondary data into various sources as obtained from the studies. 


\subsection{Phase 3: Life Cycle Impact Assessment}

In life cycle impact assessment (LCIA), the significance of a product system's potential environmental impacts, based on life cycle inventory results, is evaluated using LCIA. The LCIA consists of several elements: classification, characterization, normalization, and weighting. Of these four elements, normalization and weighting are considered optional, while the first two are mandatory elements in LCIA [10] (Figure 13). As shown in Figure 14, all 74 reviewed studies completed the classification and characterization phases, whereas 14 studies completed normalization and 10 completed weighting. Few studies included the waiting stage since it is optional and challenging.

The first step is classification, which involves identifying the impact assessment method. The most common standard method was the CML with various versions, such as CML 2 baseline 2000 V2/world, developed by the Center for Environmental Studies, and CML 2000 produced by the Center of Environmental Science of Leiden University. The second most common methods were ISO 14044 (2006), ISO (2000), and ISO 14040, followed by many other methods, such as IPCC 2001 GWP 100, proposed by the Intergovernmental Panel on Climate Change. For more information about the methods used in the studies, see Figure 15. The model used to calculate the impact is determined by the impact category the author intends to examine. As a result, LCA, ISO, and IPCC were the most commonly used impact methods since they provide categorization factors for ecotoxicity and climate change, which were among the criteria used to select articles for this review.

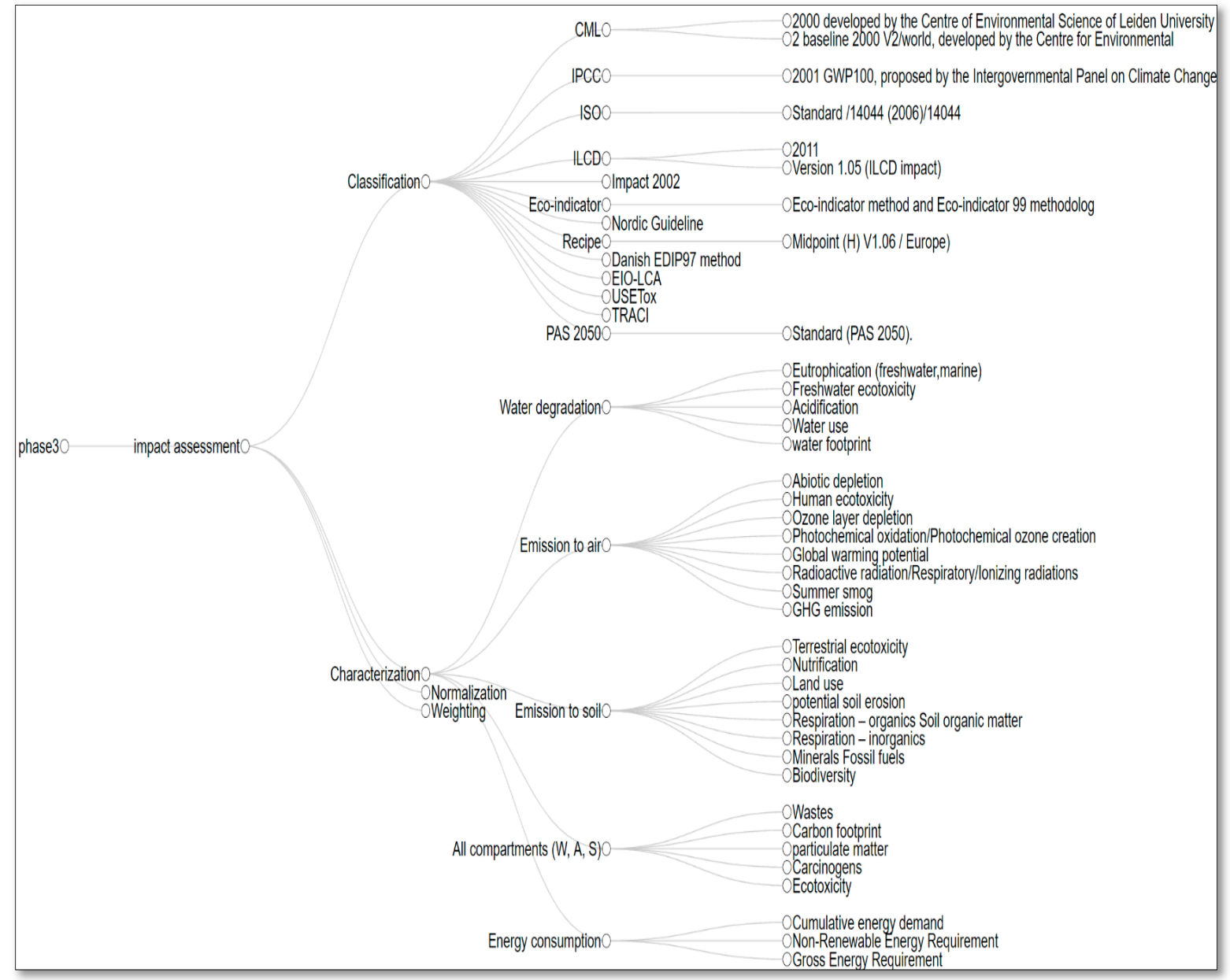

Figure 13. Phase 3 (impact assessment) of life cycle assessment (LCA). 


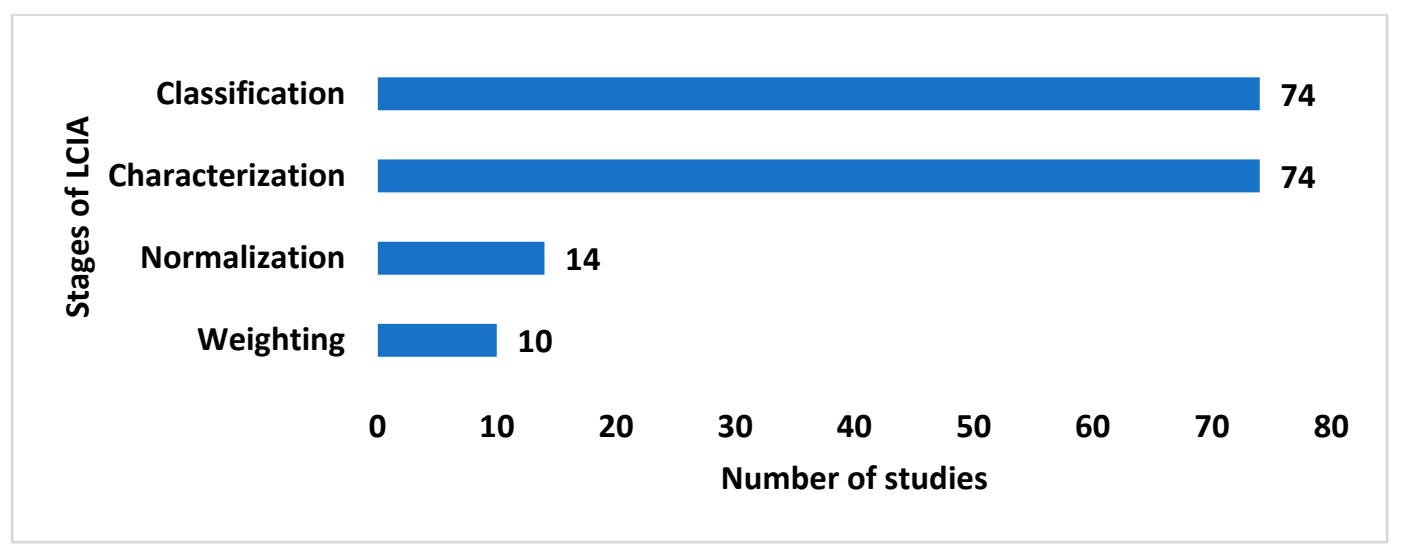

Figure 14. Quantitative and qualitative representation of the frequency of components of the LCIA phase in the reviewed studies.

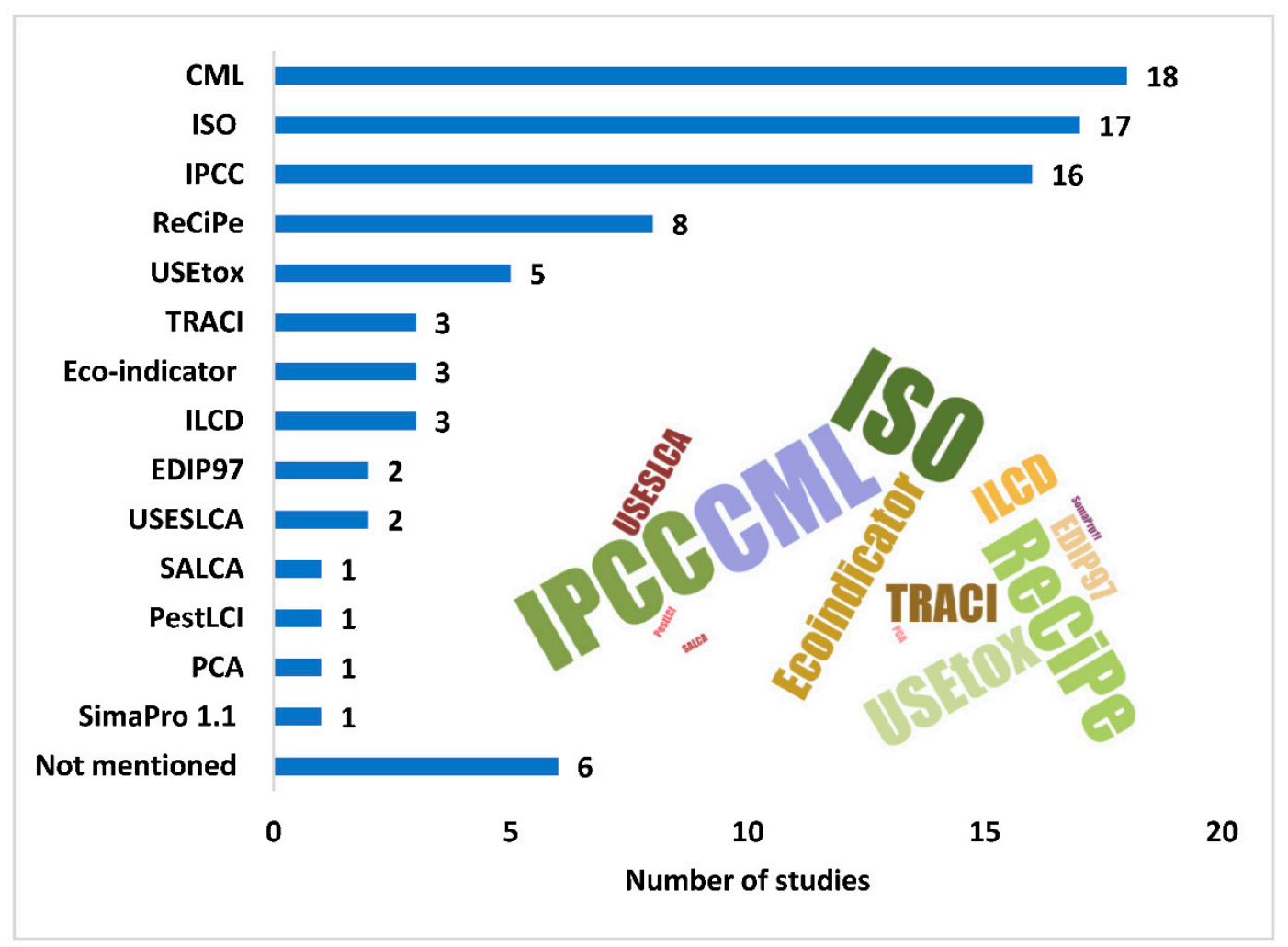

Figure 15. LCIA methods obtained from the literature review denoted by means of bar plots and a world cloud (classification).

Choosing the correct method for the LCA's impact assessment stage depends on the impact category under investigation. Each method has categories; for example, CML 2000 has 10 environmental impact categories: abiotic depletion, global warming, ozone layer depletion, human toxicity, freshwater aquatic ecotoxicity, marine aquatic ecotoxicity, terrestrial ecotoxicity, photochemical oxidation, acidification, and eutrophication.

In the process to quantify the impact of a procedure or material used, impact categories are first chosen, followed by quantifying environmental impact in each impact category using the equivalency approach. This process is termed "characterization" [10]. Characterization includes the emissions to air, soil, and water, as represented in Figure 16. The most prevalent impact categories in the 74 papers were human toxicity and ecotoxicity, with 48 and 41 studies, respectively. Moreover, 34 studies included global warming potential as an effect category, whereas marine pollution (26 articles), freshwater aquatic ecotoxicity 
(23 articles), and acidification potential (22 articles) were topics of the remaining studies (Figure 16).

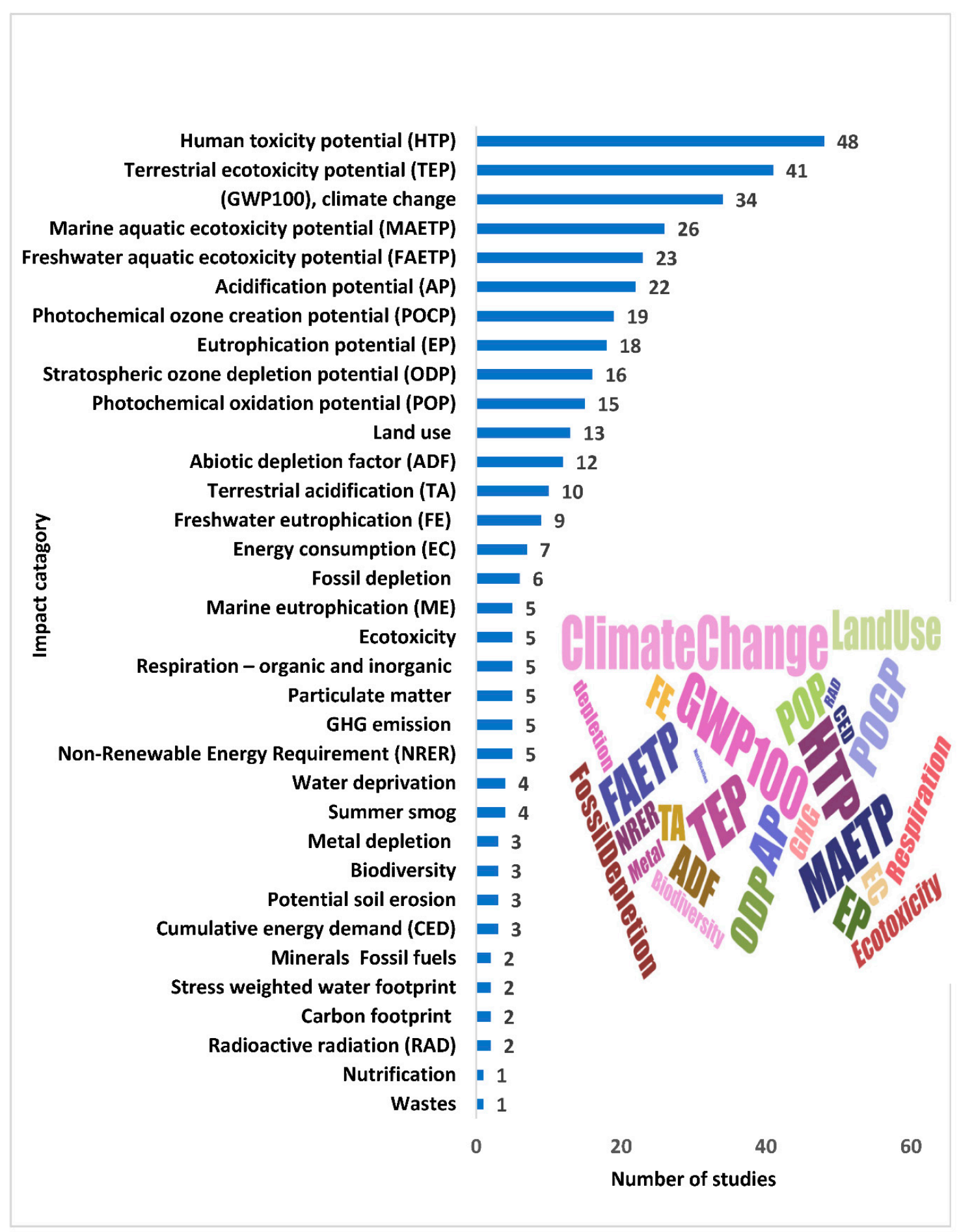

Figure 16. Illustration of LCIA impact categories from the literature (characterization).

\subsection{Phase 4: Life Cycle Interpretation/Recommendation Options}

The primary purpose of interpretation, which is the last phase of the LCA, is to use the inventory results and impact assessment analysis to evaluate the starting point for product improvement. The starting point is to understand the process tree and then identify the key issues, i.e., the key processes, materials, activities, components, or even 
life cycle stages in developing a product. The primary purpose is followed up with improvement recommendations to find more environmentally friendly designs and/or process modification. Studies applied dominance analysis and marginal analysis to identify the key issues. The dominant aspects of the inventory table may be revealed by studying the environmental elements of a process matrix. An arbitrarily chosen criterion, such as "contribution greater than $1 \%$ of the total impact", can be applied in identifying key issues from the matrix. Marginal analysis illustrates the changes in the process to which the intervention, effect, or index is most sensitive. In theory, marginal analysis is a powerful tool in determining product improvement options $[8,26]$.

Many studies stated that, for a complete understanding of the significant driver of the impacts, it is necessary to include all stages and material used through a product's life cycle, which is very challenging due to a lack of information and databases. However, depending on the aim of the LCA research, the literature review revealed a number of critical concerns, such as emissions from chemical and energy usage, the cultivation method used, land-use problems, and consumption waste.

Furthermore, studies in the literature proposed several recommendations for improving the agri-food system and reducing environmental consequences. One of them was adhering to the EPA and USDA pesticide and fertilizer guidelines. A frequent proposal was to use agricultural waste as animal feed. The most common request, however, was to enhance production without increasing inputs (Figure 17).

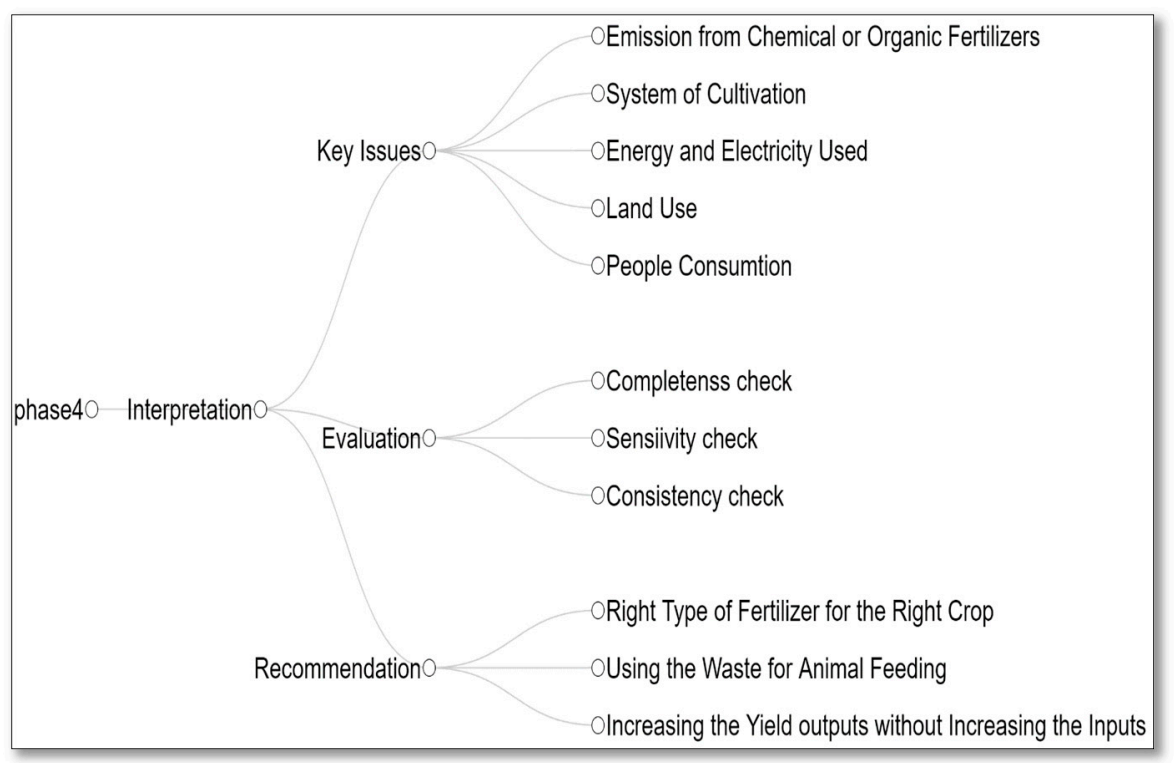

Figure 17. Phase 4 (interpretation/recommendation) of life cycle assessment (LCA).

\section{Discussion}

The present study reviewed articles related to the environmental impacts of agricultural production in LCA assessment. The main steps in conducting an LCA are defining the purpose of the study and boundary stages involved in the analysis, collecting the data of the inventory phase, estimating the impact of the involved process and used material, and then identifying the key issues, followed up with improvement recommendations. Most studies followed these steps, and some of them had common impact categories. However, implementing LCA is challenging and necessitates meticulous data collection.

\subsection{Choice of Time, Spatial Domain, and Elementary Flows in LCA}

Nearly $17 \%$ of studies did not mention the temporal scale of their analyses, depicting the inherent limitation of ISO 14040/ISO 14044 in considering the time period of evolution and process variations pertaining to diverse impact categories. The highest temporal resolution obtained from the literature was seasonal ( $4 \%$ of studies). The choice of time 
in LCA depends on the spatial and temporal scale of the impact categories considered. For example, the temporal scale of ecotoxicity varies from hours to years. On the other hand, ecotoxicity impacts have multiple transport pathways such as air, water, and soil emissions with diverse temporal scales. Establishing a time frame for the evaluation in LCA is challenging, as both very lengthy and very short periods of assessment are not practicable depending on the topic of the LCA. Extremely short timescales violate the concept of intergenerational equality, whereas extremely long ones marginalize short-term actions, lowering the incentive to act [27]. Consequently, care should be taken when defining the temporal scale of inventory flows.

About half of the studies ( $49 \%$ ) used secondary data collection for the LCA, acquiring data from websites and previous studies. The studies that constituted primary datasets were fewer due to the trouble of obtaining data at the desired spatial/temporal resolution for the inventory flows. The selection of impact categories and spatial domains (Figure 16) clearly reflects a preference for secondary datasets. The major categories studied were human toxicity potential and terrestrial ecotoxicity (the primary contributor being agricultural pesticide emissions). Studies used the approximated characterization factor from models for a particular spatial and temporal horizon to assess the potential impacts. Multimedia chemical exposure models such as CalTOX [28], USES-LCA [28,29], IMPACT 2002 [30], and USEtox [31] can provide the time-dependent concentrations of a chemical in the environmental compartments of air, soil, water, plants, and sediments. The potential impacts are characterized on the basis of the chemical's fate in an environmental partition and its effect.

\subsection{Impact Assessment}

The quantity of the input material at each stage of the crop production chain can reduce GHG, as well as emissions, including energy use (diesel, fuel, electricity) both on farm (crop production, machinery use) and off farm (transportation, refrigeration). Additional emissions include fertilizer production and use $\left(\mathrm{N}, \mathrm{P}_{2} \mathrm{O}_{5}, \mathrm{~K}_{2} \mathrm{O}\right)$, pesticide use (fungicide, herbicide, insecticide), raw material production and transportation, packaging production, and disposal (Table A2). These sources of emissions contribute to environmental impacts in various ways, including human toxicity, terrestrial toxicity, freshwater toxicity, aquatic toxicity, global warming, and acidification (Figure 16). It has been demonstrated that low-input crops have minimal impacts, but high-input crops have high impacts [32]. Furthermore, the type of input can affect the rate of the impacts. For example, replacing Thomas slag with triple superphosphate reduced the toxicity associated with the presence of heavy metals [33]. Simultaneously, replacing urea with ammonium nitrate reduced the influence of fertilization on eutrophication and acidity induced by ammonia volatilization [34].

\subsection{LCA as a Tool in Environmental Policy Decisions}

In order to achieve the population demand in the future, increasing food production is not the only pathway to increase food availability. Increased food production necessitates either more land or increased fertilizer and pesticide use on current arable land, with negative environmental consequences such as elevated GHG emissions, biodiversity loss, water contamination, and soil erosion [35]. That explains why, among the LCA papers, the most common target audiences were policymakers and producers, whereby policymakers regulate new policies for upcoming issues and producers follow these rules. The LCA methodology can be used to identify parameters and their variability in order to assist producers, wholesale and retail consumers, and policymakers in aligning their practices and purchasing decisions with low-carbon goals. LCA can also be used to analyze different production systems in order to quantify differences in input consumption and environmental consequences. The key parameters and their variability are then addressed to offer stakeholder metrics for evaluating and aligning their agricultural processes, purchasing decisions, and policies to optimize production supply chains. 


\subsection{Challenges in Collecting the Information and Limitations}

Obtaining each LCA component from the reviewed studies is not simple for the reader due to the authors' descriptive and nonexhaustive approach. Section 3 shows that diverse communities can benefit from this study on a local, international, and global scale. Hence, the author could have used a table or a flow chart to present the flow of components and stages to summarize the four phases and their components to enable the reader to focus on helpful information.

Another challenge is to identify what information needs to be included in the phases of the LCA. One of the essential characteristics of phase one of the LCA is using a functional unit; some authors mentioned it in the goal section while others mentioned it in the scope section. Noticeably, studies with an economic purpose often did not clearly report the functional unit.

The necessity of incorporating all production processes and their input materials, analyzing all phases to understand the environmental effect, and obtaining an optimal outcome from the LCA analysis of food production systems was emphasized by researchers. However, that is neither possible nor practical because of data limitations and cost restrictions [10]. Accordingly, the minor influential stages were excluded. Hence, most studies focused on a single phase of the food production chain. For example, some studies focused on the cultivation phase because they considered that the food production system's environmental impact mainly comes from farming activities.

The literature review did not focus on a specific region or a crop. Consequently, many studies appeared while searching using the keywords. Therefore, we included 74 articles related to LCA in agricultural production in general, as well as GHG emissions and ecotoxicity as an LCA impact category.

\subsection{Assumptions Used, Benefits, and Recommendations}

The LCA of crops along a food supply chain can provide helpful information from an economic, social, and environmental perspective. Using the LCA, stakeholders can better understand the energy, water, and material input and evaluate the outputs' environmental impacts. Thus, they can regulate new policies and use modern practices to improve the production supply chains.

A substantial understanding of each phase of the LCA is required to present an accurate food product's environmental impact. This paper clearly explains the LCA's major components that can serve as a primer for the scientific community. Specifically, because LCA is a systematic tool that allows for analyzing a product throughout its life cycle, LCA is used to study the economic value and importance from the local and global perspectives.

If the final product's functional unit is introduced at either the goal or the scope stage, the study results would be unaffected from our perspective. However, we recommend illustrating the input's measurement unit and the outputs while illustrating the production scope, followed by a table of units to be more readable for the audience to understand at which stage the inputs are being used and to represent the elementary flows. Defining the system boundary determines the impact pathway for an impact category that links the elementary flows from inventory to the endpoint of analysis. It is clear that the system boundary processes need to be defined according to the study's goal and the impact category. Furthermore, the functional unit must be clearly defined to explain the elementary flows from inventory to the endpoint. It is essential to know the impact category that the LCA aims to estimate, which processes are related to it, and their cause-effect relationships. The impact assessment studies were mostly conducted in the European sector since most models and databases are suited for European agri-food products.

\subsection{Research Gaps}

The information obtained from the literature sheds light on some of the future research needs: (a) the impact of land use on GHG emissions [36], (b) LCA applications based on irrigation techniques using solar energy dealing with waste streams [37], (c) LCA of processed and homegrown vegetables [38], (d) packaging of foods with eco-design 
solutions [8], and (e) applications of LCA in organic agricultural practices, fertilization practices, mulching and milling techniques, and achievable production yields [39]. Some studies have called for more LCA applications in non-European and non-OECD countries to make their agri-food sector more environmentally friendly [40]. Therefore, it is understood that LCA can be used to make the agri-food supply chain more sustainable.

The inventory flows obtained from the present review point to the inter-dependency of three sectors in LCA: energy, food, and water. Consequently, policymakers can use LCA as a tool to spot the crucial areas that need improvisation within the framework of the food-energy-water nexus. Moreover, it is imperative to understand the drivers of environmental policy for selecting an environmentally friendly agri-food supply system. The regional variation of this nexus calls for more regional LCA assessments based on the allocation of resources. More research is needed to explore future scenarios [41] that drive resource consumption and policy design for long-term sustainability utilizing the LCA framework.

Funding: This material was based on work partially supported by the USDA-National Institute of Food and Agriculture's Evans-Allen Project, grant 11979180/2016-01711 and 1890 Institution Capacity Building grants 2017-38821-26405, the National Science Foundation under grant No. 1735235, awarded as part of the National Science Foundation Research Traineeship as well as the Saudi Arabian Cultural Mission (SACM) under grant No. KSA10009393.

Institutional Review Board Statement: Not applicable.

Informed Consent Statement: Not applicable.

Data Availability Statement: Data sharing does not apply to this article as no new data were created or analyzed in this study.

Conflicts of Interest: The authors declare no conflict of interest.

\section{Appendix A}

Table A1. Common aims in the selected studies.

\begin{tabular}{lll}
\hline Aim & Type of Aim & Studies \\
\hline Evaluate the impact of all or most stages of FSC & Descriptive & {$[12,38,42-69]$} \\
\hline Determine environmental differences of different cultivation options & Comparative & {$[24,36,39,70-77]$} \\
\hline Estimate the impact of energy consumption & Descriptive & {$[42,43,48,50,78-80]$} \\
\hline Investigate the impact of different fertilization rates and type & Comparative & {$[34,70,81-84]$} \\
\hline $\begin{array}{l}\text { Quantify impacts associated during cultivation cycle of a crop using } \\
\text { life cycle analysis }\end{array}$ & Descriptive & {$[32,85-88]$} \\
\hline Investigate the impact of different pesticide rates and type & Comparative & {$[28,89-92]$} \\
\hline Evaluate the suitability of LCA & Descriptive & {$[34,93,94]$} \\
\hline Compare the energy and GHG of regional and national scale & Comparative & {$[40,95,96]$} \\
\hline Compare the impact of the cultivation of different crops & Comparative & {$[97-99]$} \\
\hline Provide datasets on several agricultural products & Descriptive & {$[47,100]$} \\
\hline Compare two different methods & Comparative & {$[101-103]$} \\
\hline
\end{tabular}


Table A2. Inventory data of the selected studies.

\begin{tabular}{|c|c|c|c|c|c|c|c|}
\hline$\#$ & Reference & Data Source & Practice & Input & Unit & Output & Unit \\
\hline 1 & [42] & $\begin{array}{l}\text { Primary data } \\
\text { Not mentioned how }\end{array}$ & $\begin{array}{l}\text { Field preparation } \\
\text { Seeding } \\
\text { Post seeding } \\
\text { weed control } \\
\text { Creation of } \\
\text { irrigation ditches } \\
\text { Irrigation } \\
\text { Irrigation } \\
\text { Supporting with } \\
\text { reeds } \\
\text { Fertilization } \\
\text { Plant protection } \\
\text { Harvest } \\
\text { Life cycle inventory data (per } 1 \mathrm{t} \text { of } \\
\text { beans produced) and (per } 1 \text { ha } \\
\text { cultivated) }\end{array}$ & $\begin{array}{l}\text { Diesel } \\
\text { Seeds } \\
\text { Manure } \\
\text { Water (electricity) } \\
\text { Herbicides, insecticides, fungicides } \\
\mathrm{N} \text { fertilizer, } \mathrm{P}_{2} \mathrm{O}_{5}, \mathrm{~K}_{2} \mathrm{O} \\
\text { Manure cattle, sheep } \\
\text { Seaweeds } \\
\text { Land occupation }\end{array}$ & $\begin{array}{l}60 \mathrm{kw} \text { tractor } \\
60 \mathrm{kw} \text { tractor } \\
\mathrm{kg} \\
\mathrm{kg} \\
\text { ton } \\
\text { ton } \\
\mathrm{m}^{2} / \text { year }\end{array}$ & $\begin{array}{l}\text { Emissions to air, water, and soil) } \\
\text { harvested beans }\end{array}$ & $\mathrm{kg}$ \\
\hline 2 & [43] & $\begin{array}{l}\text { Primary data } \\
\text { (real farm) } \\
\text { Secondary data } \\
\text { (previous studies) }\end{array}$ & $\begin{array}{l}\text { Cultivation and crop } \\
\text { Orange transport } \\
\text { Selection and washing } \\
\text { Primary extraction }\end{array}$ & $\begin{array}{l}\text { Fertilizers: } \mathrm{N}_{,} \mathrm{P}_{2} \mathrm{O}_{5}, \mathrm{~K}_{2} \mathrm{O} \\
\text { Water } \\
\text { Diesel } \\
\text { HDPE bins } \\
\text { Electric energy } \\
\text { Water } \\
\text { Recycled water }\end{array}$ & $\begin{array}{l}\mathrm{kg} \\
\mathrm{MJ} \\
\mathrm{kg} \\
\mathrm{MJ} \\
\mathrm{kg} \\
\mathrm{MJ} \\
\mathrm{kg}\end{array}$ & $\begin{array}{l}\mathrm{CO}_{2}, \mathrm{CO}, \mathrm{NO}_{x}, \mathrm{SO}_{2}, \mathrm{~N}_{2} \mathrm{O}, \mathrm{NH}_{3} \\
\text { Oranges } \\
\text { Wastes (leaves, rejected, citrus) } \\
\text { Wastewater } \\
\text { purification plant } \\
\text { Scraps }\end{array}$ & $\begin{array}{l}\mathrm{kg} \\
\mathrm{kg} \\
\mathrm{kg} \\
\mathrm{kg} \\
\mathrm{kg} \\
\mathrm{kg} \\
\mathrm{kg}\end{array}$ \\
\hline
\end{tabular}


Table A2. Cont.

\begin{tabular}{|c|c|c|c|c|c|c|c|}
\hline$\#$ & Reference & Data Source & Practice & Input & Unit & Output & Unit \\
\hline 3 & [44] & $\begin{array}{l}\text { Primary data } \\
\text { (Interview) } \\
\text { secondary data } \\
\text { (Databases) }\end{array}$ & $\begin{array}{l}\text { Crop management practices } \\
\text { Maintenance of watering canals } \\
\text { Bank management } \\
\text { Plowing } \\
\text { Fertilizing } \\
\text { Harrowing } \\
\text { Sowing } \\
\text { Application of } \\
\text { plant protection } \\
\text { products } \\
\text { Harvesting } \\
\text { Fertilizers } \\
\text { Cuoio torrefatto } \\
(12 \% \mathrm{~N}) ; \\
\text { ORVET } 8 \text { (8\% N); } \\
\text { Urea (46\% N); } \\
\text { Calce Fosfopotassica } \\
\left.\text { (8\% } \mathrm{P}_{2} \mathrm{O}_{5}-22 \% \mathrm{~K}_{2} \mathrm{O}-20 \% \mathrm{CaO}\right) ; \\
\text { Complesso } \\
\left(18 \% \mathrm{~N}-36 \% \mathrm{~K}_{2} \mathrm{O}\right) ; \\
\text { ORVET } \\
\left(10 \% \mathrm{~N}-5 \% \mathrm{P}_{2} \mathrm{O}_{5}-15 \% \mathrm{~K}_{2} \mathrm{O}\right) ; \\
\text { Complesso } \\
\left.\text { (11\% } \mathrm{N}-12 \% \mathrm{P}_{2} \mathrm{O}_{5}-36 \% \mathrm{~K}_{2} \mathrm{O}\right) \\
\text { Pesticides } \\
\text { Gulliver } \\
\text { Londax } 60 \mathrm{DF}-\mathrm{Square}_{6} 60 \mathrm{WDG} \\
\text { Pull } 52 \mathrm{DF} \\
\text { Sunrice } \\
\text { Karmex } \\
\text { Buggy-Clinic } 360 \\
\text { Stratos ultra } \\
\text { Aura } \\
\mathrm{K}-\mathrm{Othrine} \\
\text { Dipterex } \\
\text { Heteran } \\
\text { Nominee } \\
\text { Rifit } \\
\text { Cannicid-Poladan }\end{array}$ & $\begin{array}{l}\text { Excavation hydraulic digger } \\
\text { Ploughing } \\
\text { Tillage, plowing } \\
\text { Fertilizing, by broadcaster } \\
\text { Tillage, harrowing, by rotary harrow } \\
\text { Sowing } \\
\text { Application of plant protection } \\
\text { products, by field sprayer } \\
\text { Combine harvesting } \\
12 \% \mathrm{~N}, 46 \% \mathrm{~N}, 21 \% \mathrm{P}_{2} \mathrm{O}_{5}, 50 \% \mathrm{~K}_{2} \mathrm{O}\end{array}$ & $\begin{array}{l}\mathrm{m}^{3} \\
\text { ha } \\
\text { ha } \\
\text { ha } \\
\text { ha } \\
\text { ha } \\
\mathrm{kg} / \mathrm{ha}\end{array}$ & $\begin{array}{l}\text { Direct field emissions } \\
\left(\mathrm{CH}_{4}, \mathrm{NH}_{3} \text {, etc.) }\right. \\
\text { Indirect emissions from combustion } \\
\text { delivered refined rice } \\
\text { Rice byproducts: } \\
\text { husk, flour, broken } \\
\text { grains, green grains }\end{array}$ & $\mathrm{kg}$ \\
\hline
\end{tabular}


Table A2. Cont.

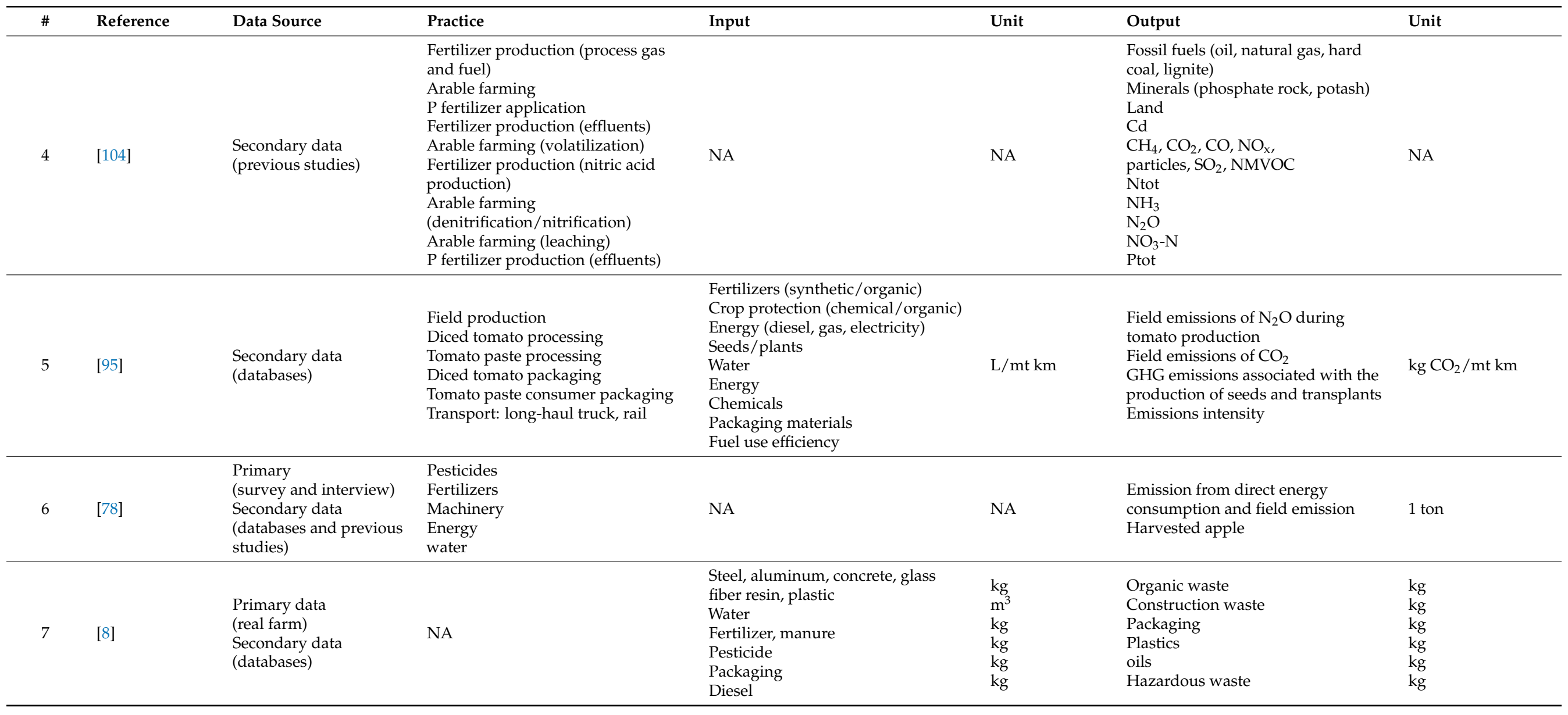


Table A2. Cont.

\begin{tabular}{|c|c|c|c|c|c|c|c|}
\hline$\#$ & Reference & Data Source & Practice & Input & Unit & Output & Unit \\
\hline 8 & [45] & $\begin{array}{l}\text { Primary data } \\
\text { (interview) } \\
\text { Secondary data } \\
\text { (databases) }\end{array}$ & $\begin{array}{l}\text { Motion of tractors } \\
\text { Conveying and unloading } \\
\text { Optical selection } \\
\text { Washing } \\
\text { Peeling } \\
\text { Crushing and pulping for the juice } \\
\text { Sorting } \\
\text { Can filling and pasteurization } \\
\text { Water purification } \\
\text { Palletizing } \\
\text { Irrigation } \\
\text { Tomato fertilization } \\
\text { Plant protection } \\
\text { Tomato fruit transport } \\
\text { Packaging }\end{array}$ & $\begin{array}{l}\text { Diesel } \\
\text { Electricity Natural gas } \\
\text { Water } \\
\mathrm{N}, \mathrm{P}_{2} \mathrm{O}_{5}, \mathrm{~K}_{2} \mathrm{O} \\
\text { Insecticide, fungicide } \\
\text { Tin can, label, carton tray, plastic } \\
\text { film, pallet, box for transport, plastic } \\
\text { boxe }\end{array}$ & $\begin{array}{l}\mathrm{kg} \\
\mathrm{kWh} / \mathrm{can} \\
\mathrm{kWh} / \mathrm{can} \\
\mathrm{m}^{3} / \mathrm{Can} \\
\mathrm{kg} \\
\mathrm{L} \\
\mathrm{g} / \mathrm{can}\end{array}$ & $\begin{array}{l}\text { The resulting impact was provided } \\
\text { as output. }\end{array}$ & NA \\
\hline 9 & [46] & $\begin{array}{l}\text { Primary data } \\
\text { (Surveys }\end{array}$ & $\begin{array}{l}\text { Resources } \\
\text { Raw materials and fossil fuels } \\
\text { Electric and thermal energy }\end{array}$ & $\begin{array}{l}\text { Occupation, permanent crop, fruit, } \\
\text { extensive } \\
\text { Transformation, to permanent crop, } \\
\text { fruit, extensive } \\
\text { Transformation, from pasture and } \\
\text { meadow } \\
\text { Water, process, unspecified natural } \\
\text { origin } \\
\text { Fertilizer } \mathrm{N}, \mathrm{P}_{2} \mathrm{O}_{5}, \mathrm{~K}_{2} \mathrm{O} \\
\text { Pesticides } \\
\text { Planting } \\
\text { Irrigating } \\
\text { Pesticide treatments } \\
\text { Transport } \\
\text { Power saw } \\
\text { Petrol unleaded at a refinery } \\
\text { Diesel at refinery } \\
\text { Lubricating oil } \\
\text { Sawmill } \\
\text { Transport, lorry 16-32 ton, } \\
\text { EURO } \\
\text { Orchard end of life }\end{array}$ & $\begin{array}{l}\text { ha } \cdot \text { year } \\
\text { ha } \\
\text { ha } \\
\mathrm{m}^{3} \\
\text { ton } \\
\text { ton } \\
\mathrm{ha} \\
\mathrm{m}^{3} \\
\mathrm{ha} \\
\mathrm{kton} \cdot \mathrm{km} \\
\mathrm{p} \\
\mathrm{kg} \\
\mathrm{kg} \\
\mathrm{kg} \\
\mathrm{p} \\
\text { ton } \cdot \mathrm{km} \\
\mathrm{p}\end{array}$ & $\begin{array}{l}\text { Emission in water } \\
\text { Nitrogen, total } \\
\text { Phosphorus, total } \\
\text { Potassium } \\
\text { Waste treatments } \\
\text { Disposal, hazardous waste, } \\
\text { 25\% water, to hazardous } \\
\text { waste incineration }\end{array}$ & $\begin{array}{l}\text { ton } \\
\text { ton } \\
\text { ton } \\
\mathrm{kg}\end{array}$ \\
\hline
\end{tabular}


Table A2. Cont.

\begin{tabular}{|c|c|c|c|c|c|c|c|}
\hline$\#$ & Reference & Data Source & Practice & Input & Unit & Output & Unit \\
\hline 10 & [47] & $\begin{array}{l}\text { Primary data } \\
\text { (interview) } \\
\text { Secondary data } \\
\text { (databases) }\end{array}$ & $\begin{array}{l}\text { Fuels, fertilizers, pesticides, water } \\
\text { use, agricultural } \\
\text { machinery models and use, yield, } \\
\text { harvest schedule, distance and } \\
\text { means of transport to the packing } \\
\text { facility. }\end{array}$ & NA & NA & $\begin{array}{l}\text { Air emission } \\
\text { Water and soil waste }\end{array}$ & NA \\
\hline 11 & [48] & $\begin{array}{l}\text { Primary data } \\
\text { (Survey) } \\
\text { Secondary data } \\
\text { (databases) }\end{array}$ & $\begin{array}{l}\text { Life cycle inventory data for } \\
\text { greenhouse tomato and cucumber } \\
\text { (per } 1 \text { ton of produced } \\
\text { crop). } \\
\text { Energy coefficients of different } \\
\text { inputs and output used }\end{array}$ & $\begin{array}{l}\text { Machinery } \\
\text { Labor } \\
\text { Diesel fuel } \\
\text { Electricity } \\
\text { Natural gas } \\
\text { Nitrogen } \\
\text { Phosphate } \\
\text { Potassium } \\
\text { Sul } \\
\text { Farmyard manure } \\
\text { Pesticides } \\
\text { Water for irrigation } \\
\text { Plastic } \\
\text { 1. Machinery } \\
\text { Tractor, self-propelled } \\
\text { Stationary } \\
\text { Equipment implemented, machinery } \\
\text { 2. Human labor } \\
\text { 3. Natural gas } \\
\text { 4. Diesel fuel } \\
\text { 5. Biocide } \\
\text { Herbicide, fungicide, insecticide } \\
\text { 6. Fertilizers: } \mathrm{N}, \mathrm{P}_{2} \mathrm{O}_{5}, \mathrm{~K}_{2} \mathrm{O} \\
\text { 7. Micro (M) } \\
\text { 8. Farmyard: manure } \\
\text { 9. Water for Irrigation } \\
\text { 10. Electricity } \\
\text { 11. Seeds }\end{array}$ & $\begin{array}{l}\mathrm{kg} \\
\mathrm{h} \\
\mathrm{L} \\
\mathrm{kWh} \\
\mathrm{m}^{3} \\
\mathrm{~kg} \\
\mathrm{~kg} \\
\mathrm{~kg} \\
\mathrm{~kg} \\
\mathrm{~kg} \\
\mathrm{~kg} \\
\mathrm{~m}^{3} \\
\mathrm{~kg} \\
\mathrm{~kg} \cdot y e a r \\
\mathrm{~kg} \cdot y e a r \\
\mathrm{~h} \\
\mathrm{~m}^{3} \\
\mathrm{~L} \\
\mathrm{~kg} \\
\mathrm{~kg} \\
\mathrm{~kg} \\
\mathrm{~kg} \\
\mathrm{~m}^{3} \\
\mathrm{kWh} \\
\mathrm{kg}\end{array}$ & Tomato/cucumber & $\mathrm{kg}$ \\
\hline
\end{tabular}


Table A2. Cont.

1- Preliminary considerations

Doses of fertilizing products applied

2- Stage of compost production

$$
\text { (CP) }
$$

Collection and transport of the

organic waste

Industrial composting process

Biofilter characteristics and gaseous

emissions

3- Stage of mineral fertilizer

$$
\text { production (FP) }
$$

4- Stage of compost transport

- Stage of mineral fertilizers transport (FT)

Primary data

(real farm)

12 [70]

(databases)

Stage of cultivation $(\mathrm{Cu})$

Fertigation infrastructure substage

(CuF)

Phytosanitary substances substage

(CuP)

Machinery and tools substage (CuM)

Irrigation substage (CuI)

Post-application emissions sub-stag

(CuE)

Nursery plants substage ( $\mathrm{CuN})$

Management of waste generated in

the cultivation stage

\section{7- $\quad$ Greenhouse (G)}

Greenhouse structure substage (GS)

Greenhouse management substage

Avoided burdens of dumping

OFMSW and BA in landfill
Fertilizer application

Compost

$\mathrm{HNO}_{3}, \mathrm{KNO}_{3}, \mathrm{KPO}_{4} \mathrm{H}_{2}, \mathrm{~K}_{2} \mathrm{SO}_{4} \quad \mathrm{~g} \cdot \mathrm{m}^{-2}$

Nitrogen application organic, $\quad \mathrm{g} \cdot \mathrm{m}^{-2}$

mineral $\quad \mathrm{g} \cdot \mathrm{m}^{-2}$

Irrigation water $\quad \mathrm{m}^{3} \cdot \mathrm{FU}^{-1}$

Per area $\quad \mathrm{t}^{\mathrm{h} \mathrm{ha}^{-1}}$

er ton tomato

Commercial yield, Total yield

Tomato average diameter

Tomato average weight

Greenhouse (GH)

Commercial yield

Total yield diameter

$\mathrm{mm}$

$\mathrm{g}$

$\cdot h a^{-1}$

tha

T MAL

Outputs of the composting process

in the industrial composting

plant of Castelldefels

Greenhouse gases

Tomato average weight

Trucks 
Table A2. Cont.

\begin{tabular}{|c|c|c|c|c|c|c|c|}
\hline$\#$ & Reference & Data Source & Practice & Input & Unit & Output & Unit \\
\hline 13 & [71] & $\begin{array}{l}\text { Secondary data } \\
\text { (both) }\end{array}$ & $\begin{array}{l}\text { Wheat life cycle inputs } \\
\text { Transport }\end{array}$ & $\begin{array}{l}\text { N, P: conv } \\
\text { Pesticide: conv } \\
\text { Phosphate rock: org } \\
\text { Manure: org } \\
\text { Diesel (org and conv) } \\
\text { Gasoline (org and conv) } \\
\text { Truck, rail transport }\end{array}$ & $\begin{array}{l}\mathrm{kg}, \mathrm{kg} \mathrm{P} \\
\mathrm{kg} \\
\mathrm{kg} \text { of } \\
\text { manure } \mathrm{P} \\
\mathrm{L} \\
\mathrm{L} \\
\mathrm{t} \mathrm{km}\end{array}$ & $\begin{array}{l}\text { Baking, } \\
\text { packaging, } \\
\text { and sales } \\
\text { Wheat } \\
\text { Flour }\end{array}$ & $\mathrm{kg}$ \\
\hline 14 & [39] & $\begin{array}{l}\text { Secondary data } \\
\text { (Both) }\end{array}$ & NA & $\begin{array}{l}\text { Average yield per cultural cycle } \\
\text { Specific area } \\
\text { Water } \\
\text { Organic fertilizers } \\
\text { Crop residues (durum wheat) } \\
\text { Manure } \\
\text { Foliar nitrogenous fertilizer } \\
\text { Differentiated and prolonged release } \\
\text { nitrogenous fertilizer } \\
\text { Mineral fertilizers } \\
\text { Controlled release NPK fertilizer } \\
\text { (14-7-14) } \\
\text { NPK complex fertilizer } \\
\text { Total nutrient supply } \\
\mathrm{N} \text { (organic fertilizers) } \\
\mathrm{N} \text { (mineral fertilizers) } \\
\mathrm{N} \text { (total) } \\
\left.\mathrm{P} \text { (total, as } \mathrm{P}_{2} \mathrm{O}_{5}\right) \\
\left.\mathrm{K} \text { (total, as } \mathrm{K}_{2} \mathrm{O}\right) \\
\text { Pesticides (active substances) } \\
\text { Benfluralin (herbicide) } \\
\text { Propyzamide (herbicide) } \\
\text { Boscalid (fungicide) } \\
\text { Pyraclostrobin (fungicide) } \\
\text { Cyprodinil (fungicide) } \\
\text { Fludioxonil (fungicide) } \\
\text { Deltamethrin (insecticide) } \\
\text { Spinosad (insecticide) } \\
\text { Black LDPE mulching film (35 mm; } \\
\text { 28 g/m²) }\end{array}$ & $\begin{array}{l}\mathrm{t} \\
\mathrm{m}^{2} \\
\mathrm{~m}^{3} \\
\mathrm{t} \\
\mathrm{t} \\
\mathrm{kg} \\
\mathrm{kg} \\
\mathrm{kg} \\
\mathrm{kg} \\
\mathrm{kg} \\
\mathrm{kg} \\
\mathrm{kg} \\
\mathrm{kg} \\
\mathrm{kg} \\
\mathrm{kg} \\
\mathrm{kg} \\
\mathrm{kg} \\
\mathrm{kg}\end{array}$ & $\begin{array}{l}\text { To air: } \mathrm{NH}_{3}, \mathrm{NO}_{\mathrm{x}} \\
\text { Groundwater: } \mathrm{NO}_{3}^{-} \\
\text {Surface waters: }\left(\mathrm{PO}_{4}\right) \\
\text { Soil } \\
\text { Heavy metals }(\mathrm{Cd}, \mathrm{Cr}, \mathrm{Cu}, \mathrm{Ni}, \mathrm{Pb} \text {, } \\
\mathrm{Zn}) \\
\text { Pesticides (active substances) }\end{array}$ & \\
\hline
\end{tabular}


Table A2. Cont.

\begin{tabular}{|c|c|c|c|c|c|c|c|}
\hline$\#$ & Reference & Data Source & Practice & Input & Unit & Output & Unit \\
\hline 15 & [96] & $\begin{array}{l}\text { Secondary data } \\
\text { (databases) }\end{array}$ & $\begin{array}{l}\text { Fertilizer production } \\
\text { Pesticide production } \\
\text { Production of greenhouse } \\
\text { infrastructure }\end{array}$ & $\begin{array}{l}\text { Mineral fertilizer N } \\
\text { Mineral fertilizer P } \\
\text { Mineral fertilizer K } \\
\text { Manure compost } \\
\text { Organic fertilizer } \\
\text { Steel } \\
\text { Aluminum } \\
\text { Glass } \\
\text { Plexiglas } \\
\text { Plastic } \\
\text { Iron } \\
\text { Concrete } \\
\text { Rockwool }\end{array}$ & 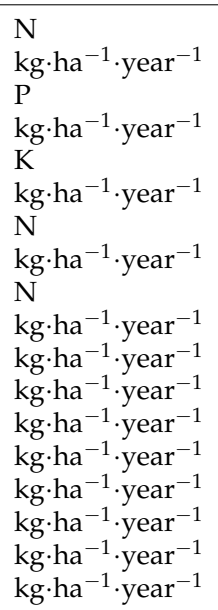 & $\begin{array}{l}\text { Machine use } \\
\text { Energy demand heating } \\
\text { changes in soil organic carbon }\end{array}$ & $\begin{array}{l}\mathrm{h} \mathrm{ha}^{-1} \\
\mathrm{GJ}^{-1} \text { year } \\
\mathrm{N}_{2} \mathrm{O} \text { emissions } \\
\text { direct } \mathrm{N}_{2} \mathrm{O} \\
\text { emissions indirect } \\
\text { Humus } \\
\text { sequestration }\end{array}$ \\
\hline 16 & [81] & $\begin{array}{l}\text { Primary data } \\
\text { (real farm) }\end{array}$ & NA & $\begin{array}{l}\mathrm{N} \text { min in the soil in spring } \\
\text { Mineral } \mathrm{N} \text { fertilizer rate } \\
\text { Atmospheric } \mathrm{N} \text { deposition } \\
\text { Net } \mathrm{N} \text { mineralization during } \\
\text { vegetation } \\
\text { Mineralization of } \mathrm{N} \text { from sugar beet } \\
\text { leaves (easily degradable part) } \\
\text { Mineralization of } \mathrm{N} \text { from sugar beet } \\
\text { leaves (slowly degradable part) }\end{array}$ & NA & $\begin{array}{l}\mathrm{NH}_{3} \text { volatilization } \\
\mathrm{N}_{2} \mathrm{O} \text { emission } \\
\mathrm{N} \text { removal with beets } \\
\mathrm{N} \text { content of leaves } \\
\mathrm{N} \text { uptake of winter wheat in autumn }\end{array}$ & One ton of grain \\
\hline 17 & [49] & $\begin{array}{l}\text { Primary data } \\
\text { (interview) } \\
\text { Secondary data } \\
\text { (databases) }\end{array}$ & $\begin{array}{l}\text { Greenhouse } \\
\text { Training system } \\
\text { Irrigation system }\end{array}$ & $\begin{array}{l}\text { Low-density } \\
\text { Polyethylene } \\
\text { Sawn timber } \\
\text { Steel } \\
\text { Wire } \\
\text { Polyethylene } \\
\text { Sawn timber } \\
\text { Wire } \\
\text { Polyethylene } \\
\text { Polyvinylchloride }\end{array}$ & $\begin{array}{l}\mathrm{k} \\
\mathrm{m}^{3} \\
\mathrm{~kg} \\
\mathrm{~kg} \\
\mathrm{~kg} \\
\mathrm{~m}^{3} \\
\mathrm{~kg} \\
\mathrm{~kg} \\
\mathrm{~kg}\end{array}$ & $\begin{array}{l}\text { Fresh tomato } \\
\text { Air emissions } \\
\mathrm{NH}_{3} \\
\mathrm{~N}_{2} \mathrm{O}-\mathrm{N} \\
\mathrm{NO}_{x}-\mathrm{N} \\
\mathrm{Water} \mathrm{emissions} \\
\mathrm{N}-\mathrm{NO}_{3}\end{array}$ & $\begin{array}{l}\mathrm{t} \\
\mathrm{kg} \cdot \mathrm{ha}^{-1} \\
\mathrm{~kg} \cdot \mathrm{ha}^{-1}\end{array}$ \\
\hline
\end{tabular}


Table A2. Cont.

\begin{tabular}{|c|c|c|c|c|c|c|c|}
\hline$\#$ & Reference & Data Source & Practice & Input & Unit & Output & Unit \\
\hline 18 & [50] & $\begin{array}{l}\text { Primary data } \\
\text { (real farm) } \\
\text { Secondary data } \\
\text { (previous studies and } \\
\text { databases) }\end{array}$ & $\begin{array}{l}\text { Cultivation } \\
\text { and crop } \\
\text { Primary process } \\
\text { (citrus selection and washing, } \\
\text { extraction) } \\
\text { Secondary process } \\
\text { (refining; centrifugation) } \\
\text { Secondary process } \\
\text { (refining; pasteurization and cooling) } \\
\text { Concentration and cooling } \\
\text { Packaging and storage } \\
\text { Transport of final products }\end{array}$ & $\begin{array}{l}\text { Fertilizers } \\
\text { Water } \\
\text { Diesel } \\
\text { Electric energy } \\
\text { Water } \\
\text { Recycled water } \\
\text { Water-oil emulsion } \\
\text { Electric energy } \\
\text { Cooling water } \\
\text { Raw juice } \\
\text { Methane } \\
\text { Electric energy } \\
\text { Steam } \\
\text { Electric energy } \\
\text { Methane } \\
\text { Steam } \\
\text { Cooling water } \\
\text { electric energy } \\
\text { Essential oil } \\
\text { Electric energy } \\
\text { Natural juice } \\
\text { Concentrated juice } \\
\text { HFO, Diesel }\end{array}$ & NA & $\begin{array}{l}\text { Air emissions } \\
\text { Amount of citrus fruit } \\
\text { Wastes (scraps, leaves, rejected } \\
\text { citrus) } \\
\text { Wastewater to a purification plant } \\
\text { Scraps to pressing } \\
\text { process } \\
\text { Essential oil to packaging and } \\
\text { storage } \\
\text { Wet wastes } \\
\text { Wastewater to purification } \\
\text { plant } \\
\text { Natural and concentrated juice } \\
\text { Concentrated juice }\end{array}$ & NA \\
\hline 19 & [40] & $\begin{array}{l}\text { Secondary data } \\
\text { (previous studies) }\end{array}$ & $\begin{array}{l}\text { (larvae/fingerlings, fertilizers, and } \\
\text { feeds). }\end{array}$ & NA & NA & nitrogen and phosphorus emissions & NA \\
\hline
\end{tabular}


Table A2. Cont.

\begin{tabular}{|c|c|c|c|c|c|c|c|}
\hline$\#$ & Reference & Data Source & Practice & Input & Unit & Output & Unit \\
\hline 20 & [51] & $\begin{array}{l}\text { Primary data } \\
\text { (reports) } \\
\text { Secondary data } \\
\text { (databases) }\end{array}$ & $\begin{array}{l}\text { Land use } \\
\text { Pesticides } \\
\text { Fertilizer use } \\
\text { Fuel use } \\
\text { Seed use } \\
\text { Sun use } \\
\text { Agr. operations } \\
\text { Lime hydrated } \\
\text { Cane } \\
\text { Cane transport } \\
\text { River water } \\
\text { Air } \\
\text { Softened water } \\
\text { Ammonium sulfate } \\
\text { Sulfuric acid } \\
\text { Yeast } \\
\text { Transport of filter cake } \\
\text { Transport of ashes }\end{array}$ & $\begin{array}{l}\text { Diuron, Glyphosate, Gesapox 80, } \\
\text { MSMA 72, Amine Salt, Isoctilic ester } \\
\text { 48, Asulox 40, Goxone, Amigan 65, } \\
\text { Merlin 75, Sulfatante 90, Unspecified } \\
\text { Urea, } \mathrm{P}_{2} \mathrm{O}_{5}, \mathrm{~K}_{2} \mathrm{O} \\
\text { Diesel } \\
\text { Cane seed } \\
\text { Solar energy } \\
\text { Harvesting } \\
\text { Fertilizing } \\
\text { Planting } \\
\text { Irrigating } \\
\text { NaOH } 50 \% \text { in } \mathrm{H}_{2} \mathrm{O} \\
\mathrm{HCl} 30 \% \text { in } \mathrm{H}_{2} \mathrm{O}\end{array}$ & $\begin{array}{l}\mathrm{ha} / \text { year } \\
\mathrm{kg} / \text { ha } \cdot \text { year } \\
\mathrm{kg} / \mathrm{ha} \cdot \text { year } \\
\mathrm{kg} / \mathrm{ha} \cdot \text { year } \\
\mathrm{kg} / \mathrm{h} \cdot \text { year } \\
\mathrm{kg} / \mathrm{ha} \cdot \text { year } \\
\mathrm{kg} / \mathrm{ha} \cdot \text { year } \\
\mathrm{kg} / \text { day } \\
\mathrm{kg} / \text { ha } \cdot \text { year } \\
\mathrm{GJ} / \text { day } \\
\mathrm{h} \text { /year } \\
\mathrm{ha} / \text { year } \\
\mathrm{ha} / \text { year } \\
\mathrm{ha} / \text { year } \\
\mathrm{t} / \text { day } \\
\mathrm{t} / \text { day } \\
\mathrm{t} / \text { day } \\
\mathrm{t} / \text { day } \\
\mathrm{km} \\
\mathrm{t} / \text { day } \\
\mathrm{t} / \text { day } \\
\mathrm{t} / \text { day } \\
\mathrm{t} / \text { day } \\
\mathrm{t} / \text { day } \\
\mathrm{t} / \text { day } \\
\mathrm{km}\end{array}$ & $\begin{array}{l}\text { Cane products } \\
\text { Cane } \\
\text { Agr. Wastes } \\
\text { Emissions } \\
\mathrm{N}_{2} \mathrm{O} \\
\mathrm{N} \text { total to water } \\
\text { Pesticides to water Pesticides to soil } \\
\text { Sugar } \\
\text { Molassesa } \\
\text { Electr. to networka } \\
\text { Alcohol } \\
\text { Biogas } \\
\text { Ash }\left(\mathrm{P}_{2} \mathrm{O}_{5} \text { equiv.) }\right. \\
\text { Ash }\left(\mathrm{K}_{2} \mathrm{O} \text { equiv.) }\right. \\
\text { Sludge/wastewater/cake }(\text { urea } \\
\text { equiv.) } \\
\text { Sludge/wastewater/cake }\left(\mathrm{P}_{2} \mathrm{O}_{5}\right. \\
\text { equiv.) } \\
\text { Sludge/wastewater/cake }\left(\mathrm{K}_{2} \mathrm{O}\right. \\
\text { equiv.) } \\
\text { Emissions to air } \\
\text { PM } \\
\text { Nitrogen oxides } \\
\text { Emissions to water } \\
\text { Wastewater } \\
\text { Inorganic solids } \\
\text { Total nitrogen } \\
\text { Chemical oxygen demand } \\
\text { Total phosphorus } \\
\text { Emissions to soil } \\
\text { Ashes } \\
\text { Filter cake }\end{array}$ & $\begin{array}{l}\text { t/day } \\
t / \text { day } \\
\mathrm{kg} / \text { day } \\
\mathrm{kg} / \text { day } \\
\mathrm{kg} / \text { day } \\
\mathrm{kg} / \text { day } \\
\mathrm{t} / \text { day } \\
\mathrm{t} / \text { day } \\
\mathrm{G} / \text { day } \\
\mathrm{t} / \text { day } \\
\mathrm{t} / \text { day } \\
\mathrm{t} / \text { day } \\
\mathrm{t} / \text { day } \\
\mathrm{t} / \text { day } \\
\mathrm{t} / \text { day } \\
\mathrm{t} / \text { day } \\
\mathrm{t} / \text { day } \\
\mathrm{t} / \text { day } \\
\mathrm{t} / \text { day } \\
\mathrm{t} / \text { day } \\
\mathrm{t} / \text { day } \\
\mathrm{t} / \text { day } \\
\mathrm{t} / \text { day } \\
\mathrm{t} / \text { day } \\
\mathrm{t} / \text { day }\end{array}$ \\
\hline 21 & [52] & $\begin{array}{l}\text { Primary data } \\
\text { (interview) } \\
\text { Secondary data } \\
\text { (databases) }\end{array}$ & $\begin{array}{l}\text { Seed production and transport } \\
\text { Fertilizer protection and transport } \\
\text { Pesticide production and transport } \\
\text { Machinery protection and } \\
\text { maintenance } \\
\text { Energy carriers and protection }\end{array}$ & NA & NA & $\begin{array}{l}\text { Emission to air and water } \\
\text { Solid emission }\end{array}$ & NA \\
\hline
\end{tabular}


Table A2. Cont.

\begin{tabular}{|c|c|c|c|c|c|c|c|}
\hline$\#$ & Reference & Data Source & Practice & Input & Unit & Output & Unit \\
\hline 22 & [53] & $\begin{array}{l}\text { Secondary data } \\
\text { (databases) }\end{array}$ & $\begin{array}{l}\text { Cultivation: } \\
\text { Plastic cover } \\
\text { Greenhouse } \\
\text { Transportation: } \\
\text { small truck, truck, sea, pre-cooling, } \\
\text { and storage }\end{array}$ & $\begin{array}{l}\text { fuel consumption, refrigeration, } \\
\text { driving }\end{array}$ & $\begin{array}{l}\mathrm{L} / \mathrm{t} \mathrm{km} \\
\mathrm{kWh} / \mathrm{m}^{3} / \text { year }\end{array}$ & $\begin{array}{l}\text { Waste management }\left(\mathrm{CO}_{2} \text { emission, }\right. \\
\mathrm{t} / \mathrm{t} \mathrm{t} \\
\text { Paper, board, plastics } \\
\mathrm{CO}_{2} \text { emission from packaging, } \\
\text { transportation, and storage } \\
\text { Transportation } \\
\text { Farm to packing house } \\
\text { Packinghouse to wholesale }\end{array}$ & $\begin{array}{l}\mathrm{kg} / \mathrm{t} \\
\mathrm{kg} / \mathrm{t} \\
\mathrm{kg} / \mathrm{t} \cdot \mathrm{km} \\
\mathrm{kg} / \mathrm{t}\end{array}$ \\
\hline 23 & [54] & $\begin{array}{l}\text { Secondary data } \\
\text { (databases) }\end{array}$ & $\begin{array}{l}\text { Cattle manure } \\
\text { Fuel use for various types of driving } \\
\text { machinery and for different loads } \\
\text { Low power } \\
\text { Medium power } \\
\text { High power } \\
\text { Combine } \\
\text { Willow harvester }\end{array}$ & $\begin{array}{l}\mathrm{N}, \mathrm{P}_{2} \mathrm{O}_{5}, \mathrm{~K}_{2} \mathrm{O} \text { fertilizer } \\
\text { Slurry } \\
\text { Power }\end{array}$ & $\begin{array}{l}\mathrm{mg} / \mathrm{kg} \\
\mathrm{mg} / \mathrm{kg} \\
\mathrm{kw}\end{array}$ & $\begin{array}{l}\text { Willow } \\
\text { Straw } \\
\text { Wheat }\end{array}$ & $\begin{array}{l}\mathrm{mg} / \mathrm{kg} \\
\mathrm{mg} / \mathrm{kg} \\
\mathrm{mg} / \mathrm{kg}\end{array}$ \\
\hline 24 & [82] & $\begin{array}{l}\text { Secondary data } \\
\text { (both) }\end{array}$ & $\begin{array}{l}\text { Yields for main products } \\
\text { Straw yields and crop residues } \\
\text { Moisture content } \\
\text { Quantity of seed } \\
\text { Use of machinery (number of passes) } \\
\text { Sowing and harvest date } \\
\text { Quantity of fertilizers } \\
\text { Types of fertilizers in integrated } \\
\text { systems } \\
\text { Types of fertilizers in organic } \\
\text { systems } \\
\text { Pesticide applications } \\
\text { Chemical seed dressing } \\
\text { Machinery classes } \\
\text { Tractor harvester Trailer machinery, } \\
\text { tillage } \\
\text { Slurry tank }\end{array}$ & $\begin{array}{l}\text { Steel, unalloyed } \\
\text { Steel, alloyed } \\
\text { Other metals } \\
\text { Rubber } \\
\text { Plastics } \\
\text { Others (glass, paints, etc.) }\end{array}$ & $\mathrm{NA}$ & $\begin{array}{l}\text { Ammonia emissions } \\
\text { Nitrate leaching } \\
\text { P-emissions } \\
\mathrm{N}_{2} \mathrm{O} \text { emissions } \\
\text { Heavy-metal emissions } \\
\text { Pesticide applications } \\
\text { Tractor combustion emissions }\end{array}$ & NA \\
\hline
\end{tabular}


Table A2. Cont.

\# Reference

Data Source

Practice

nput

Fertilizers and lime

Nitrogen fertilizer (urea and

diammonium phosphate)

Phosphate fertilizer (diammonium

phosphate)

Potassium fertilizer (potassium

chloride)

Inventory of agricultural inputs

Agrochemical types and application

rates

Irrigation water intake

Secondary data

25

(both)

Pesticides: Clopyralid, Haloxyfop
Fuel consumption in agricultural

operations

Operating rate in machinery

Agricultural machinery type

Seed yield (calcic carbonate)

Picloram, Glyphosate, Linuron,

Thiophanate-methyl, Prochloraz

Seed for sowing

Irrigation requiremen

Irrigation water intake

Diesel consumption: plowing,

harrowing, crushing

sowing, spraying, weeding,

hilling/fertilizing harvest

Tractor for field operations

Seed yield
Unit

Output

Unit $\mathrm{kg} \mathrm{N}$

$\mathrm{kg} \mathrm{P}_{2} \mathrm{O}_{5}$

$\mathrm{kg} \mathrm{KaCO}_{2} \mathrm{O}$
$\mathrm{kg} \mathrm{CaCO}$

$\mathrm{kg}$

$\mathrm{kg}$

$\mathrm{m}^{3}$

$\mathrm{kg}$

$\mathrm{kg}$

$\mathrm{kg}$
Ammonia $\left(\mathrm{NH}_{3}\right)$

Nitrates $\left(\mathrm{NO}_{3}\right)$

Nitrous oxide $\left(\mathrm{N}_{2} \mathrm{O}\right)$

Nitrogen oxides $\left(\mathrm{NO}_{\mathrm{x}}\right)$

Phosphates $\left(\mathrm{PO}_{4}\right)$

Carbon dioxide $\left(\mathrm{CO}_{2}\right)$

Glyphosate (main pesticide in

rapeseed)

inuron (main pesticide in

sunflower) $\mathrm{kg} / \mathrm{xkg}$

$\mathrm{kg} / \mathrm{xkg}$

$\mathrm{kg} / \mathrm{xkg}$

$\mathrm{kg} / \mathrm{xkg}$

$\mathrm{kg} / \mathrm{xkg}$

$\mathrm{kg} / \mathrm{xkg}$

$\mathrm{kg} / \mathrm{xkg}$

$\mathrm{kg} / \mathrm{xkg}$

kg/xkg 
Table A2. Cont.

[55] $\begin{aligned} & \text { Secondary data } \\ & \text { (databases) }\end{aligned}$

Secondary d
(databases)

Inventory data on wheat production

(1995-2011, year ${ }^{-1}$ ).

Production costs

Seed

Chemical fertilizers

Purchased manure

Pesticides 49858

Fossil fuels 14760

Electricity

Land improvement and irrigation

Agricultural services

Buildings

Agricultural machinery

Fossil fuels

Heavy oil

Diesel oil

Wheat grown in paddy fields and

Kerosene

Wheat grown in upland fields

Motor oil

Premixed fuel

Calcium carbonate

fertilizer

Nitrogen balance

Chemical fertilizers

Purchased manure

Atmospheric deposition

Wheat straw (incorporated)

Wheat

Wheat straw (total)

Denitrification

Ammonia volatilization

$$
\text { Surplus }
$$

Wheat straw

Air-emission sources included fossil

fuel combustion, fertilizer

application, and crop residue

$\begin{array}{ll}\text { yen } \cdot \mathrm{ha}^{-1} & \text { incorporation } \\ \mathrm{L} \cdot \mathrm{ha}^{-1} & \text { Emissions in fossil fuel combustion }\end{array}$

$\mathrm{L} \cdot \mathrm{ha}^{-1}$

were calculated using the $\mathrm{CO}_{2}, \mathrm{CH}_{4}$,

and $\mathrm{N}_{2} \mathrm{O}$ emission factors

and the $\mathrm{NO}_{\mathrm{x}}$ and SOx emission

factors

The $\mathrm{CO}_{2}$ emission factor of calcium

carbonate fertilizer on a weight

the basis was $12 \%$ 
Table A2. Cont.

\begin{tabular}{|c|c|c|c|c|c|c|c|}
\hline$\#$ & Reference & Data Source & Practice & Input & Unit & Output & Unit \\
\hline 27 & [105] & $\begin{array}{l}\text { Primary data } \\
\text { (real farm) } \\
\text { Secondary data } \\
\text { (previous studies and } \\
\text { databases) }\end{array}$ & $\begin{array}{l}\text { Farming } \\
\text { Irrigation } \\
\text { Soil management } \\
\text { Pest treatment } \\
\text { Fertilization } \\
\text { Pruning } \\
\text { Harvesting } \\
\text { Olive oil mill } \\
\text { Washing } \\
\text { Milling } \\
\text { Pressing } \\
\text { Decantation } \\
\text { Oil pomace mill } \\
\text { Pitting } \\
\text { Drying } \\
\text { Solvent extraction } \\
\text { Dysventilation and condensation }\end{array}$ & $\begin{array}{l}\text { Water } \\
\text { Pesticides } \\
\text { Fertilizers } \\
\text { Diesel } \\
\text { Lubrification oil } \\
\text { water } \\
\text { Electric energy } \\
\text { Water } \\
\text { Electric energy } \\
\text { Hexane }\end{array}$ & $\begin{array}{l}\mathrm{m}^{3} \\
\mathrm{~kg} \\
\mathrm{~kg} \\
\mathrm{~kg} \\
\mathrm{~L} \\
\mathrm{~m}^{3} \\
\mathrm{kWh} \\
\mathrm{L} \\
\mathrm{kWh} \\
\mathrm{kg}\end{array}$ & $\begin{array}{l}\text { Olive mill } \\
\text { Wastewater } \\
\text { Water from washing } \\
\text { Virgin olive } \\
\text { Exhausted pomace } \\
\text { Pomace oil }\end{array}$ & $\begin{array}{l}\mathrm{L} \\
\mathrm{L} \\
\mathrm{L} \\
\mathrm{L} \\
\mathrm{kg} \\
\mathrm{kg}\end{array}$ \\
\hline 28 & [56] & $\begin{array}{l}\text { Primary data } \\
\text { (interview) }\end{array}$ & $\begin{array}{l}\text { Fertilization } \\
\text { Pesticides } \\
\text { Packaging } \\
\text { Transportation }\end{array}$ & $\begin{array}{l}\text { N, P, K } \\
\text { Lubricating oils } \\
\text { Seeds } \\
\text { Tomatoes } \\
\text { Sugar beets } \\
\text { Tomato paste } \\
\text { Raw sugar } \\
\text { Sugar solution } \\
\text { Vinegar } \\
\text { Spice emulsion } \\
\text { Salt } \\
\text { Tomato ketchup } \\
\text { Packaging system for tomato paste } \\
\text { Packaging system for ketchup } \\
\text { Transportation } \\
\text { Shopping } \\
\text { Household phase } \\
\text { Electricity production } \\
\text { Waste management }\end{array}$ & & $\begin{array}{l}\mathrm{CH}_{4}, \mathrm{~N}_{2} \mathrm{O}, \mathrm{CO} \mathrm{NMHC} \\
\text { Biological oxygen demand (BOD) } \\
\mathrm{NO}_{\mathrm{x}} \\
\text { Other organic compounds } \\
\text { Water emissions } \\
\text { Soil emissions }\end{array}$ & $\begin{array}{l}\mathrm{kg} \text { per } \mathrm{FU} \\
\mathrm{kg} \text { per } \mathrm{FU} \\
\mathrm{g} \text { per FU } \\
\mathrm{m}^{3} \text { per FU } \\
\mathrm{kg} \text { soil per FU }\end{array}$ \\
\hline
\end{tabular}


Table A2. Cont.

\# Reference

Data Source

\section{Input}

Occupation, arable land

Plants (plugs)

$\mathrm{CO}_{2}$ from air fixed in crop

Tractor use

Diesel (for field operations)

Steel (spare parts replacement)

Labor (labor-intensive operations)

Diesel (for workers' transport)

Plastic (fleece, mulch ...

Pesticides (unspecified)

Fertilizers: N, P, K

Manure/organic fertilizers

Irrigation

Bluewater, surface water

Bluewater, groundwater

frastructure (pipes, sprinklers ... )

Electricity (pumps)

Primary input and output flow from the case study farms during broccoli cropping

Input packed broccoli to RDC

Diesel for transport to RDC

rom Spain

From the UK

Electricity RDC storage

Input packed broccoli to retailer

Inventory of retail-to-grave

processes

RDC

Retailer

Household
Diesel for transport to retailer

Electricity retailer storage and display

Solid waste from retailer to landfill

Broccoli

LDPE packaging

Diesel for solid waste transport

Input broccoli to household

Petrol for transport to household

Diesel for transport to household

Electricity home storage

Electricity cooking

Natural gas cooking

Tap water

Solid waste from household to landfill

Broccoli

LDPE packaging

Diesel for solid waste transport

Cooking wastewater to WWTP

Cooked broccoli (input to human excretion)
Unit

Output

Unit

Soil emissions (literature)

$\mathrm{kg}$

$\mathrm{CO}_{2}$ from soil

$\mathrm{CH}_{4}$ from soil

$\mathrm{NH}_{3}$ from soil

$\mathrm{NO}_{x}$ from soil

$\mathrm{N}_{2} \mathrm{O}$ from soil

$\mathrm{NO}_{3}$ from soil

$\mathrm{PO}_{4}$ from soil

Change in soil organic carbon (SOC) 
Table A2. Cont.

\begin{tabular}{|c|c|c|c|c|c|c|c|}
\hline$\#$ & Reference & Data Source & Practice & Input & Unit & Output & Unit \\
\hline 30 & [38] & $\begin{array}{l}\text { Secondary data } \\
\text { (databases) }\end{array}$ & $\begin{array}{l}\text { Data inventory for the agricultural } \\
\text { phase } \\
\text { Data inventory for the processing } \\
\text { phase (data refer to FU) }\end{array}$ & $\begin{array}{l}\text { Seeds } \\
\text { Compost from cow and horse } \\
\text { manure } \\
\text { Fosetyl-Al } \\
\text { [Thio]carbamate-compounds } \\
\text { [Sulfonyl]urea-compounds } \\
\text { Diesel fuel } \\
\text { Water } \\
\text { Electricity for irrigation } \\
\text { LDPE film (greenhouse) } \\
\text { Land } \\
\text { Salad (Valerianella locusta) } \\
\text { Salad } \\
\text { Electricity } \\
\text { Water } \\
\text { Sodium hypochlorite } \\
\text { PP film }\end{array}$ & $\begin{array}{l}\mathrm{Mg} \\
\mathrm{g} \\
\mathrm{mg} \\
\mathrm{mg} \\
\mathrm{mg} \\
\mathrm{g} \\
\mathrm{dm}^{3} \\
\mathrm{kWh} \\
\mathrm{mg} \\
\mathrm{m}^{2} \\
\mathrm{~g} \\
\mathrm{~g} \\
\mathrm{kWh} \\
\mathrm{dm}^{3} \\
\mathrm{mg} \\
\mathrm{g}\end{array}$ & $\begin{array}{l}\text { Emissions to air } \\
\text { Carbon dioxide } \\
\text { Carbon monoxide } \\
\text { Nitrogen oxides } \\
\text { Particulate hydrocarbons } \\
\text { Dinitrogen monoxide } \\
\text { Ammonia } \\
\text { Benfluralin } \\
\text { Fosetyl-Al } \\
\text { Propamocarb } \\
\text { Emissions to water } \\
\text { Benfluralin } \\
\text { Fosetyl-Al } \\
\text { Propamocarb } \\
\text { Emissions to soil } \\
\text { Benfluralin } \\
\text { Fosetyl-Al } \\
\text { Propamocarb } \\
\text { Salad bag (130 g) } \\
\text { Salad scraps } \\
\text { PP film waste } \\
\text { Wastewater }\end{array}$ & $\begin{array}{l}\mathrm{g} \\
\mathrm{mg} \\
\mathrm{mg} \\
\mathrm{mg} \\
\mathrm{mg} \\
\mathrm{mg} \\
\mathrm{mg} \\
\mathrm{mg} \\
\mathrm{mg} \\
\mathrm{mg} \\
\mathrm{mg} \\
\mathrm{mg} \\
\mathrm{mg} \\
\mathrm{mg} \\
\mathrm{mg} \\
\mathrm{p} \\
\mathrm{g}\end{array}$ \\
\hline 31 & [98] & $\begin{array}{l}\text { Secondary data } \\
\text { (previous studies) }\end{array}$ & NA & NA & NA & NA & NA \\
\hline
\end{tabular}


Table A2. Cont.

\begin{tabular}{|c|c|c|c|c|c|c|c|}
\hline$\#$ & Reference & Data Source & Practice & Input & Unit & Output & Unit \\
\hline 32 & [73] & $\begin{array}{l}\text { Primary data } \\
\text { (interview) } \\
\text { Secondary data } \\
\text { (databases) }\end{array}$ & $\begin{array}{l}\text { Main characteristics of the life cycle } \\
\text { inventory of the studied } \\
\text { conventional (Con) and organic } \\
\text { (Org) groups of fruit tree orchards } \\
\text { crops in Spain. Data refer to } 1 \text { ha } \\
\text { and year unless otherwise stated }\end{array}$ & $\begin{array}{l}\text { Drip irrigation } \\
\text { Surface irrigation } \\
\text { Water use } \\
\text { Electricity } \\
\text { Presence of cover crops } \\
\text { Machinery use } \\
\text { Fuel consumption } \\
\text { Mulching plastic } \\
\text { Mineral nitrogen } \\
\text { Mineral phosphorus } \\
\text { Mineral potassium } \\
\text { Manure } \\
\text { Slurry } \\
\text { Cover crop seeds } \\
\text { Other organic fertilizers } \\
\text { Total carbon inputs } \\
\text { Total nitrogen inputs Synthetic } \\
\text { pesticides } \\
\text { Sulfur } \\
\text { Copper } \\
\text { Paraffin } \\
\text { Natural pesticides } \\
\text { Production } \\
\text { Yield }\end{array}$ & $\begin{array}{l}\% \text { of cases } \\
\% \text { of cases } \\
\mathrm{m}^{3} \\
\mathrm{kWh} \\
\% \\
\mathrm{~h} \\
\mathrm{~L} \\
\mathrm{~kg} \\
\mathrm{~kg} \mathrm{~N} \\
\mathrm{~kg} \mathrm{P}_{2} \mathrm{O}_{5} \\
\mathrm{~kg} \mathrm{~K}_{2} \mathrm{O} \\
\mathrm{mg} \\
\mathrm{mg} \\
\mathrm{kg} \\
\mathrm{kg} \\
\mathrm{kg} \\
\mathrm{kg} \\
\mathrm{kg} \text { active matter } \\
\mathrm{kg} \\
\mathrm{kg} \\
\mathrm{kg}\end{array}$ & $\begin{array}{l}\text { Soil emissions } \\
\text { Direct nitrous oxide } \\
\text { Indirect nitrous oxide } \\
\text { Methane } \\
\text { Carbon }\end{array}$ & $\begin{array}{l}\mathrm{kg} \mathrm{N}_{2} \mathrm{O} \\
\mathrm{kg} \mathrm{N}_{2} \mathrm{O} \\
\mathrm{kg} \mathrm{CH}_{4} \\
\mathrm{kgC}\end{array}$ \\
\hline
\end{tabular}


Table A2. Cont.

\begin{tabular}{|c|c|c|c|c|c|c|c|}
\hline$\#$ & Reference & Data Source & Practice & Input & Unit & Output & Unit \\
\hline 33 & [57] & $\begin{array}{l}\text { Secondary data } \\
\text { (previous studies) }\end{array}$ & $\begin{array}{l}\text { LCI to produce a single oil palm } \\
\text { seedling }\end{array}$ & $\begin{array}{l}\text { Electricity } \\
\text { Diesel } \\
\text { Polybag } \\
\text { Water } \\
\text { Fertilizer: } \mathrm{N}_{2} \mathrm{P}_{2} \mathrm{O}_{5}, \mathrm{~K}_{2} \mathrm{O} \\
\text { Thiocarbamate } \\
\text { Pyrethroid } \\
\text { Organophosphate } \\
\text { Dithiocarbamate } \\
\text { Unspecified pesticide } \\
\text { Urea/sulfonylurea } \\
\text { Glyphosate } \\
\text { Transportation Van }\end{array}$ & $\begin{array}{l}\mathrm{kWh} \\
\mathrm{L} \\
\mathrm{kg} \\
\mathrm{L} \\
\mathrm{kg} \\
\mathrm{kg} \\
\mathrm{kg} \\
\mathrm{kg} \\
\mathrm{kg} \\
\mathrm{kg} \\
\mathrm{kg} \\
\mathrm{kg} \\
\mathrm{tkm}\end{array}$ & $\begin{array}{l}\text { Emissions to air } \\
\mathrm{NH}_{3} \\
\mathrm{~N}_{2} \mathrm{O} \\
\mathrm{NO} \\
\mathrm{N}_{2} \\
\text { Glyphosate } \\
\text { Metsulfuron-methyl } \\
\text { Glufosinate ammonium } \\
\text { Paraquat } \\
\text { Emissions to water } \\
\mathrm{NO}_{3}^{-} \\
\mathrm{PO}_{4}^{-3} \\
\mathrm{Glyphosate}^{\text {Metsulfuron-methyl }} \\
\text { Carbofuran } \\
\text { Glufosinate ammonium } \\
\text { Paraquat } \\
\text { Emissions to soil } \\
\text { Glyphosate } \\
\text { Metsulfuron-methyl } \\
\text { Carbofuran } \\
\text { Glufosinate ammonium } \\
\text { Methamidophos } \\
\text { Paraquat }\end{array}$ & $\begin{array}{l}\mathrm{kg} / \mathrm{t} \mathrm{FFB} \\
\mathrm{kg} / \mathrm{t} \text { FFB } \\
\text { Leached out and } \\
\text { runoff } \\
\mathrm{g} / \mathrm{t} \mathrm{FFB}\end{array}$ \\
\hline 34 & [58] & $\begin{array}{l}\text { Primary data } \\
\text { (real farm) } \\
\text { Secondary data } \\
\text { (databases) }\end{array}$ & $\begin{array}{l}\text { Fertilizer doses, application } \\
\text { emissions, and irrigation water (per } \\
\text { ha) for lettuce and escarole crops in } \\
\text { the open field (OF), plastic mulch } \\
\text { (PM), plastic mulch combined with } \\
\text { fleece system (PM F), and } \\
\text { greenhouse (GH) systems. } \\
\text { Characteristics of materials and } \\
\text { electricity and diesel consumption } \\
\text { (per ha) included } \\
\text { in the inventory. PY polyethylene, } \\
\text { PP polypropylene. }\end{array}$ & $\begin{array}{l}\text { Fertilizer doses } \\
\mathrm{N} \text { optimum } \\
\mathrm{P}_{2} \mathrm{O}_{5} \\
\mathrm{~K}_{2} \mathrm{O} \\
\text { Mulch } \\
\text { Fleece } \\
\text { Main pipe 1 } \\
\text { Main pipe 2 } \\
\text { Main pipe 3 } \\
\text { Secondary pipes } \\
\text { Drip irrigation pipes } \\
\text { (laterals) } \\
\text { Pumps } \\
\text { Electricity (pumps) } \\
\text { Electricity (climate system) } \\
\text { Diesel (crop management) }\end{array}$ & $\begin{array}{l}\mathrm{kg} \\
\mathrm{m}^{2} \\
\mathrm{~m}^{2} \\
\mathrm{~m} \\
\mathrm{~m} \\
\mathrm{~m} \\
\mathrm{~m} \\
\mathrm{~kg} \\
\mathrm{MJ} \\
\mathrm{MJ}\end{array}$ & $\begin{array}{l}\text { Air emissions } \\
\mathrm{NH}_{3}-\mathrm{N} \\
\mathrm{NO} 2-\mathrm{N} \\
\text { Water emissions } \\
\mathrm{NO}_{3}-\mathrm{N} \\
\text { Irrigation water }\end{array}$ & $\begin{array}{l}\mathrm{kg} \\
\mathrm{kg} \\
\mathrm{m}^{3}\end{array}$ \\
\hline
\end{tabular}


Table A2. Cont.

\begin{tabular}{|c|c|c|c|c|c|c|c|}
\hline$\#$ & Reference & Data Source & Practice & Input & Unit & Output & Unit \\
\hline 35 & [59] & $\begin{array}{l}\text { Primary data } \\
\text { (interview) }\end{array}$ & $\begin{array}{l}\text { Principal inputs involved in the } \\
\text { analysis of the "Delizie di Bosco del } \\
\text { Piemonte" production } \\
\text { chain for raspberries and giant } \\
\text { American blueberries } \\
\text { Nursery } \\
\text { Rooting } \\
\text { Mulching } \\
\text { Covering } \\
\text { Covering } \\
\text { Fertigation system Fertigation } \\
\text { system Fertigation } \\
\text { Fertigation } \\
\text { Nozzles } \\
\text { Cold storage } \\
\text { Field } \\
\text { Soil preparation } \\
\text { Soil preparation } \\
\text { Mulching } \\
\text { Total processes } \\
\text { Mulching } \\
\text { Irrigation system } \\
\text { Irrigation system } \\
\text { Irrigation } \\
\text { Irrigation } \\
\text { Base fertilization } \\
\text { Total fertilization } \\
\text { Covering } \\
\text { Covering } \\
\text { Plant protection } \\
\text { treatments } \\
\text { Post-harvesting } \\
\text { Refrigeration } \\
\text { Flow packaging } \\
\text { Flow packaging } \\
\text { Flow packaging }\end{array}$ & $\begin{array}{l}\text { Substratum } \\
\text { Black PE } \\
\text { White PE } \\
\text { Metal supports } \\
\text { PVC piping } \\
\text { PVC tubing } \\
\text { Compost mix } \\
\text { Water } \\
\text { PVC } \\
\text { Electrical energy } \\
\text { Plow or cultivator } \\
\text { Harrow } \\
\text { Bed-former } \\
\text { Diesel consumption } \\
\text { PE sheeting } \\
\text { PVC piping } \\
\text { PVC tubing } \\
\text { Water } \\
\text { Electrical energy for the well } \\
\text { Manure } \\
\text { Compost } \\
\text { White PE } \\
\text { Metal supports } \\
\text { p.a. } \\
\text { Electrical energy } \\
\text { Electrical energy } \\
\text { PE tray } \\
\text { PE wrapping }\end{array}$ & $\begin{array}{l}\mathrm{L} \cdot \mathrm{ha}^{-1} \\
\mathrm{~kg} \cdot \mathrm{ha}^{-1} \\
\mathrm{~kg} \cdot \mathrm{ha}^{-1} \\
\mathrm{~kg} \cdot \mathrm{ha}^{-1} \\
\mathrm{~kg} \cdot \mathrm{ha}^{-1} \\
\mathrm{~kg} \cdot \mathrm{ha}^{-1} \\
\mathrm{~kg} \cdot \mathrm{ha}^{-1} \\
\mathrm{~m}^{3} \cdot \mathrm{ha}^{-1} \\
\mathrm{~kg} \cdot \mathrm{ha}^{-1} \\
\mathrm{kWh} \cdot \mathrm{m}^{-3} \\
\mathrm{~h} \cdot \mathrm{ha}^{-1} \\
\mathrm{~h} \cdot \mathrm{ha}^{-1} \\
\mathrm{~h} \cdot \mathrm{ha}^{-1} \\
\mathrm{~L} \cdot \mathrm{h}^{-1} \\
\mathrm{~kg} \cdot \mathrm{ha}^{-1} \\
\mathrm{~kg} \cdot \mathrm{ha}^{-1} \\
\mathrm{~kg} \cdot \mathrm{ha}^{-1} \\
\mathrm{~m}^{3} \cdot \mathrm{ha}^{-1} \\
\mathrm{kWh} \cdot \mathrm{ha}^{-1} \\
\mathrm{t} \cdot \mathrm{ha}^{-1} \\
\mathrm{t} \cdot \mathrm{ha}^{-1} \\
\mathrm{~kg} \cdot \mathrm{ha}^{-1} \\
\mathrm{~kg} \cdot \mathrm{ha}^{-1} \\
\mathrm{~kg} \cdot \mathrm{ha}^{-1} \\
\mathrm{kWh} \cdot \mathrm{kg}^{-1} \\
\mathrm{kWh} \cdot \mathrm{kg}^{-1} \\
\mathrm{~g} \cdot \mathrm{kg}^{-1} \\
\mathrm{~g} \cdot \mathrm{kg}^{-1}\end{array}$ & $\begin{array}{l}\text { GWP (global warming potential) } \\
\text { IPCC 100a } \\
\text { Nonrenewable energy }\end{array}$ & $\begin{array}{l}\mathrm{kg} \mathrm{CO}_{2} \text { eq } \\
\text { MJ primary }\end{array}$ \\
\hline
\end{tabular}


Table A2. Cont.

\begin{tabular}{|c|c|c|c|c|c|c|c|}
\hline$\#$ & Reference & Data Source & Practice & Input & Unit & Output & Unit \\
\hline 36 & [60] & $\begin{array}{l}\text { Secondary data } \\
\text { (databases) }\end{array}$ & $\begin{array}{l}\text { Rice production } \\
\text { tillage, growing, harvest }\end{array}$ & Machines, materials & & $\begin{array}{l}\text { Rice field } \\
\text { Pollution (emissions) } \\
\text { Product, byproduct } \\
\text { Rice field product, byproduct, } \\
\text { pollution }\end{array}$ & \\
\hline 37 & [36] & $\begin{array}{l}\text { Primary data } \\
\text { (survey) } \\
\text { Secondary data } \\
\text { (previous studies and } \\
\text { databases) }\end{array}$ & & $\begin{array}{l}\text { Seed } \\
\text { Power tiller diesel fuel use } \\
\text { GHG intensity diesel fuel } \\
\text { Power tiller life expectancy } \\
\text { Power tiller weight } \\
\text { Tractor L diesel fuel/h } \\
\text { Tractor weight } \\
\text { Embodied GHG of steel } \\
\text { Bullocks } \\
\text { Allocation to straw } \\
\text { Tractors embodied emission } \\
\text { Fertilizers } \\
\text { Pesticides } \\
\text { Manure } \\
\text { Nitrogen use efficiency }\end{array}$ & 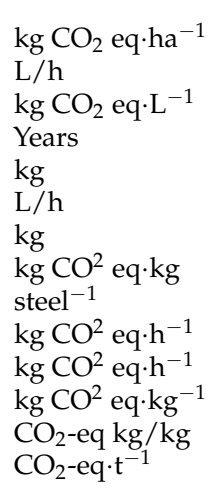 & $\begin{array}{l}\text { Methane emissions } \\
\mathrm{Nitrous}_{\text {oxide emissions }} \\
\mathrm{SRI} \mathrm{CH}_{4} \text { and } \mathrm{N}_{2} \mathrm{O} \text { emissions } \\
\text { Electricity-based emissions from } \\
\text { irrigation. } \\
\text { Embodied GHG emissions } \\
\text { associated with electricity } \\
\text { Harvest } \\
\text { Soil organic carbon }\end{array}$ & $\begin{array}{l}\mathrm{kg} \mathrm{CO}_{2} \text {-eq } \cdot \mathrm{ha}^{-1} \\
\mathrm{~kg} \mathrm{CO} \text { eq.ha }{ }^{-1} \\
\mathrm{~kg} \mathrm{CO} \text { eq.ha }{ }^{-1} \mathrm{~kg} \\
\mathrm{CO}^{2} \text { eq } \cdot \mathrm{kWh} \mathrm{h}^{-1} \\
\mathrm{GHG}^{\mathrm{emissions}} \cdot \mathrm{h}^{-1} \\
\mathrm{~kg} \mathrm{CO}_{2} \text { eq.ha }{ }^{-1}\end{array}$ \\
\hline 39 & {$[62]$} & $\begin{array}{l}\text { Primary data } \\
\text { (reports) } \\
\text { Secondary data } \\
\text { (previous studies) }\end{array}$ & $\begin{array}{l}\text { Orchard establishment inputs } \\
\text { Agricultural stage inputs } \\
\text { Retail stages inputs } \\
\text { Consumption stages inputs }\end{array}$ & $\begin{array}{l}\text { Water } \\
\text { Electricity } \\
\text { Diesel } \\
\text { Machinery } \\
\text { Materials } \\
\text { Transport }\end{array}$ & $\begin{array}{l}\mathrm{L} \\
\mathrm{kW} \\
\mathrm{kg} \\
\mathrm{kg} \\
\mathrm{kg} \\
\mathrm{tkm}\end{array}$ & $\begin{array}{l}\text { Apple } \\
\text { Peach } \\
\text { (NPK) } \mathrm{NO}_{x} \\
\mathrm{~N}_{2} \mathrm{O} \\
\text { Machinery production emissions } \\
\text { and diesel } \\
\text { consumed for machinery operations }\end{array}$ & $\begin{array}{l}\mathrm{kg} \\
\mathrm{kg}\end{array}$ \\
\hline
\end{tabular}


Table A2. Cont.

\begin{tabular}{|c|c|c|c|c|c|c|c|}
\hline$\#$ & Reference & Data Source & Practice & Input & Unit & Output & Unit \\
\hline 40 & [74] & $\begin{array}{l}\text { Secondary data } \\
\text { (both) }\end{array}$ & $\begin{array}{l}\text { Annual chemical inputs for } \\
\text { managing a mature orange grove in } \\
\text { Florida } \\
\text { Chemical mowing } \\
\text { Herbicide spray } \\
\text { Pesticide spray } \\
\text { Fertilization } \\
\text { Use of energy products for } \\
\text { undertaking various cultural } \\
\text { activities at a mature orange grove } \\
\text { in Florida } \\
\text { Site preparation } \\
\text { Management of a mature } \\
\text { orange grove }\end{array}$ & $\begin{array}{l}\text { Roundup weather max } \\
\text { Solicam } 80 \text { DF } \\
\text { Karmex WP } \\
\text { Roundup weather max } \\
\text { Prowl H20 } \\
\text { Simazine 4L } \\
\text { Roundup weather max } \\
\text { Mandate } \\
\text { Direx 4L } \\
\text { Roundup weather max } \\
\text { Spray oil } \\
\text { Copper (Kocide 3000) } \\
\text { Agrimek (if no mite resistance) } \\
\text { Zn, Mn, B } \\
\text { Lorsban 4EC } \\
\text { Copper (Kocide 3000) } \\
\text { Spray Oil } \\
\text { MgO } \\
\text { Dolomite } \\
\text { Mowing (mechanical) } \\
\text { Mowing (chemical) } \\
\text { Discing } \\
\text { Soil shaping } \\
\text { Planting } \\
\text { Mowing (mechanical) Mowing } \\
\text { (chemical) } \\
\text { Fertilization (16-0-16-4 MgO) } \\
\text { Fertilization (lime) } \\
\text { Herbicide } \\
\text { Pesticide } \\
\text { Conditioning } \\
\text { Topping } \\
\text { Hedging } \\
\text { Brush removing } \\
\text { Chopping brush } \\
\text { Dead tree removal } \\
\text { Irrigation } \\
\text { Fruit picking } \\
\text { Transporting pickers Roadsiding } \\
\text { fruit }\end{array}$ & $\begin{array}{l}\mathrm{mL} / \mathrm{ha} \\
\mathrm{kg} / \mathrm{ha} \\
\mathrm{kg} / \mathrm{ha} \\
\mathrm{mL} / \mathrm{ha} \\
\mathrm{mL} / \mathrm{ha} \\
\mathrm{mL} / \mathrm{ha} \\
\mathrm{mL} / \mathrm{ha} \\
\mathrm{mL} / \mathrm{ha} \\
\mathrm{mL} / \mathrm{ha} \\
\mathrm{mL} / \mathrm{ha} \\
\mathrm{L} / \mathrm{ha} \\
\mathrm{kg} / \mathrm{ha} \\
\mathrm{mL} / \mathrm{ha} \\
\mathrm{kg} / \mathrm{ha} \\
\mathrm{mL} / \mathrm{ha} \\
\mathrm{kg} / \mathrm{ha} \\
\mathrm{L} / \mathrm{ha} \\
\mathrm{kg} / \mathrm{ha} \\
\mathrm{kg} / \mathrm{ha}\end{array}$ & $\begin{array}{l}\text { Emission from energy use } \\
\text { Emission from material use }\end{array}$ & $\begin{array}{l}\mathrm{g} \mathrm{CO}_{2} \text { eq./FU } \\
\mathrm{g} \mathrm{CO}_{2} \text { eq./FU }\end{array}$ \\
\hline
\end{tabular}


Table A2. Cont.

\begin{tabular}{|c|c|c|c|c|c|c|c|}
\hline$\#$ & Reference & Data Source & Practice & Input & Unit & Output & Unit \\
\hline 41 & [75] & $\begin{array}{l}\text { Primary data } \\
\text { (survey) } \\
\text { Secondary data } \\
\text { (databases) }\end{array}$ & $\begin{array}{l}\text { Principal inputs involved in the } \\
\text { production and distribution chain } \\
\text { (scenarios } 1 \text { and 2) for strawberries } \\
\text { Nursery } \\
\text { Rooting } \\
\text { Mulching } \\
\text { Covering } \\
\text { Covering } \\
\text { Fertigation system Fertigation } \\
\text { system Fertigation } \\
\text { Fertigation } \\
\text { Cold storage } \\
\text { Field } \\
\text { Soil preparation } \\
\text { Soil preparation } \\
\text { Mulching } \\
\text { Total processes } \\
\text { Mulching } \\
\text { Irrigation system } \\
\text { Irrigation system } \\
\text { Irrigation } \\
\text { Irrigation } \\
\text { Base fertilization } \\
\text { Total fertilization } \\
\text { Covering } \\
\text { Covering } \\
\text { Plant protection } \\
\text { treatments } \\
\text { Post-harvesting } \\
\text { Refrigeration } \\
\text { Flow packaging } \\
\text { Flow packaging }\end{array}$ & $\begin{array}{l}\text { Substratum } \\
\text { Black PE } \\
\text { White PE } \\
\text { Metal supports } \\
\text { PVC piping } \\
\text { PVC tubing } \\
\text { Compost mix } \\
\text { Water } \\
\text { Electrical energy } \\
\text { Plow or cultivator } \\
\text { Harrow } \\
\text { Bed-former } \\
\text { Diesel consumption } \\
\text { PE sheeting } \\
\text { PVC piping } \\
\text { PVC tubing } \\
\text { Water } \\
\text { Electrical energy for the well } \\
\text { Manure } \\
\text { Compost } \\
\text { White PE } \\
\text { Metal supports } \\
\text { p.a. } \\
\text { Electrical energy } \\
\text { PE tray } \\
\text { PE wrapping }\end{array}$ & $\begin{array}{l}\mathrm{L} \cdot \mathrm{ha}^{-1} \\
\mathrm{~kg} \cdot \mathrm{ha}^{-1} \\
\mathrm{~kg} \cdot \mathrm{ha}^{-1} \\
\mathrm{~kg} \cdot \mathrm{ha}^{-1} \\
\mathrm{~kg} \cdot \mathrm{ha}^{-1} \\
\mathrm{~kg} \cdot \mathrm{ha}^{-1} \\
\mathrm{~kg} \cdot \mathrm{ha}^{-1} \\
\mathrm{~m}^{3} \cdot \mathrm{ha}^{-1} \\
\mathrm{kWh} \cdot \mathrm{m}^{-3} \\
\mathrm{~h} \cdot \mathrm{ha}^{-1} \\
\mathrm{~h} \cdot \mathrm{h}^{-1} \\
\mathrm{~h} \cdot \mathrm{ha}^{-1} \\
\mathrm{~L} \cdot \mathrm{h}^{-1} \\
\mathrm{~kg} \cdot \mathrm{ha}^{-1} \\
\mathrm{~kg} \cdot \mathrm{ha}^{-1} \\
\mathrm{~kg} \cdot \mathrm{ha}^{-1} \\
\mathrm{~m} \cdot \mathrm{ha}^{-1} \\
\mathrm{kWh} \cdot \mathrm{ha}^{-1} \\
\mathrm{t} \cdot \mathrm{ha}^{-1} \\
\mathrm{t} \cdot \mathrm{ha}^{-1} \\
\mathrm{~kg} \cdot \mathrm{ha}^{-1} \\
\mathrm{~kg} \cdot \mathrm{ha}^{-1} \\
\mathrm{~kg} \cdot \mathrm{ha}^{-1} \\
\mathrm{kWh} \cdot \mathrm{kg}^{-1} \\
\mathrm{~g} \cdot \mathrm{kg}^{-1} \\
\mathrm{~g} / \mathrm{kg}^{\mathrm{kg}}\end{array}$ & $\begin{array}{l}\text { GWP (global warming potential) } \\
\text { IPCC 100a } \\
\text { Non-renewable energy }\end{array}$ & $\begin{array}{l}\mathrm{kg} \mathrm{CO} \mathrm{CO}_{2} \mathrm{eq} \cdot \mathrm{UF}^{-1} \\
\mathrm{~kg} \mathrm{CO} \mathrm{CO}_{2} \text { eq.UF } \mathrm{UF}^{-1}\end{array}$ \\
\hline
\end{tabular}


Table A2. Cont.

\begin{tabular}{|c|c|c|c|c|c|c|c|}
\hline$\#$ & Reference & Data Source & Practice & Input & Unit & Output & Unit \\
\hline 42 & [93] & $\begin{array}{l}\text { Secondary data } \\
\text { (databases) }\end{array}$ & $\begin{array}{l}\text { Life cycle inventory data for watermelon } \\
\text { cultivation (per ha). } \\
\text { Characterization factors of inputs used in } \\
\text { watermelon production. } \\
\text { Parameters and coefficients of objective } \\
\text { functions. }\end{array}$ & $\begin{array}{l}\text { 1. Human labor (man/woman) } \\
\text { 2. Diesel fuel } \\
\text { Plowing } \\
\text { Discing } \\
\text { Ditcher } \\
\text { 3. Machinery } \\
\text { Tractor and self-propelled } \\
\text { Implement and machinery } \\
\text { 4. Fertilizers } \\
\text { Nitrogen }(\mathrm{N}) \\
\text { Phosphate }\left(\mathrm{P}_{2} \mathrm{O}_{5}\right) \\
\text { Potassium }\left(\mathrm{K}_{2} \mathrm{O}\right) \\
\text { Microelements } \\
\text { 5. Farmyard manure } \\
\text { 6. Electricity } \\
\text { 7. Chemicals } \\
\text { Fungicide } \\
\text { Insecticide } \\
\text { 8. Seeds } \\
\text { 9. Plastics } \\
\text { Machinery } \\
\text { Diesel fuel } \\
\text { Chemical fertilizers } \\
\text { (a) Urea } \\
\text { (b) Phosphate }\left(\mathrm{P}_{2} \mathrm{O}_{5}\right) \\
\text { (c) Potassium }\left(\mathrm{K}_{2} \mathrm{O}\right) \\
\text { Manure } \\
\text { Pesticides } \\
\text { Electricity } \\
\text { Plastics } \\
\text { Constanta } \\
\mathrm{N} \\
\mathrm{K}_{2} \mathrm{O} \\
\mathrm{P}_{2} \mathrm{O}_{5} \\
\text { Manure } \\
\text { Diesel } \\
\text { Electricity } \\
\text { Seed } \\
\text { Chemicals } \\
\text { Machinery } \\
\text { Plastic } \\
\text { Water }\end{array}$ & $\begin{array}{l}\mathrm{h} \\
\mathrm{L} \\
\mathrm{kg} \\
\mathrm{kg} \\
\mathrm{kg} \\
\mathrm{kWh} \\
\mathrm{kg} \\
\mathrm{kg} \\
\mathrm{kg} \\
\mathrm{kg} \\
\mathrm{L} \\
\mathrm{kg} \\
\mathrm{kg} \\
\mathrm{kg} \\
\mathrm{kWh} \\
\mathrm{kg} \\
\mathrm{kg} \\
\mathrm{kg} \\
\mathrm{kg} \\
\mathrm{kg} \\
\mathrm{L} \\
\mathrm{kWh} \\
\mathrm{kg} \\
\mathrm{kg} \\
\mathrm{kg} \\
\mathrm{kg} \\
\mathrm{MJ}\end{array}$ & $\begin{array}{l}\text { Watermelon } \\
\text { On-farm emissions } \\
\text { N fertilizer } \\
\text { Diesel fuel }\end{array}$ & $\begin{array}{l}\mathrm{kg} \\
\mathrm{kg} \\
\mathrm{MJ}\end{array}$ \\
\hline
\end{tabular}


Table A2. Cont.

\begin{tabular}{|c|c|c|c|c|c|c|c|}
\hline$\#$ & Reference & Data Source & Practice & Input & Unit & Output & Unit \\
\hline 43 & [76] & $\begin{array}{l}\text { Secondary data } \\
\text { (databases) }\end{array}$ & NA & $\begin{array}{l}\text { Mulching film for pot production } \\
\text { (PP) } \\
\text { Wind-stopper (galvanized iron) } \\
\text { Hydraulic pipe/micro pipe } \\
\text { (PEHD/PELD/PVC) } \\
\text { Taps (PEHD/PVC) } \\
\text { Tunnel cover } \\
\text { Tunnel structure (galvanized iron) } \\
\text { Poles (galvanized iron/wood) } \\
\text { Sprinklers (galvanized iron) } \\
\text { Hydraulic fittings (PE) } \\
\text { Solenoid (PVC) } \\
\text { Support canes (bamboo) } \\
\text { Black clip (PP) } \\
\text { Plates PP black wire (nylon) } \\
\text { Green thread (PVC) } \\
\text { Iron wire (galvanized iron) } \\
\text { Elastics/hooks/butterfly valve (PE) } \\
\text { Irrigation bar (aluminum) } \\
\text { Block (concrete) } \\
\text { Covering (gravel/volcanic stones) } \\
\text { Raincoat towel (PVC/PP/PEHD) } \\
\text { A chain-link fence (galvanized iron) } \\
\text { Centrifugal/submersible pump } \\
\text { (Cast iron/stainless steel) } \\
\text { Electrical panel (PEHD/copper) } \\
\text { Burlap (jute) } \\
\text { String (sisal) } \\
\text { Wire basket (iron) } \\
\text { Plastic net (PP) } \\
\text { Plastic box (PP) }\end{array}$ & NA & $\begin{array}{l}\text { Total yearly GHG emissions are } \\
\text { divided into different categories (kg } \\
\mathrm{CO}_{2} \text { eq/ } \mathrm{m}^{2} / \text { year) } \\
\text { NFS } \frac{1}{4} \text { nursery farm structure; AGS } \\
\frac{1}{4} \text { aboveground structures; IC } \frac{1}{4} \\
\text { inputs of cultivation; } \mathrm{P} \frac{1}{4} \text { packaging; } \\
\text { EFS } \frac{1}{4} \text { emissions from soil }\end{array}$ & NA \\
\hline 44 & [106] & $\begin{array}{l}\text { Secondary data } \\
\text { (databases) }\end{array}$ & NA & $\begin{array}{l}\text { Strawberry (nursery field) } \\
\text { PE punnet } \\
\text { PE plastic film } \\
\text { End-of-life } \\
\text { Transport } \\
\text { Electricity }\end{array}$ & NA & $\begin{array}{l}\text { Nonrenewable energy } \\
\text { IPCC GWP 100a }\end{array}$ & $\begin{array}{l}\mathrm{MJ} \cdot \mathrm{UF}^{-1} \\
\mathrm{~kg} \mathrm{CO} \\
2 \mathrm{eq} \cdot \mathrm{UF}^{-1}\end{array}$ \\
\hline
\end{tabular}


Table A2. Cont.

\begin{tabular}{|c|c|c|c|c|c|c|c|}
\hline$\#$ & Reference & Data Source & Practice & Input & Unit & Output & Unit \\
\hline 45 & [79] & $\begin{array}{l}\text { Primary data } \\
\text { (surveys) }\end{array}$ & $\begin{array}{l}\text { Gasoline at the refinery (US) } \\
\text { Diesel at the refinery (US) } \\
\text { Urea ammonium nitrate (UAN), (US) } \\
\text { Monoammonium phosphate (US) } \\
\text { Waxes/paraffin at the refinery (US) } \\
\text { Potassium sulfate, at regional } \\
\text { storage (Europe) } \\
\text { Mined from natural sources, only } \\
\text { transport is modeled } \\
\text { Fishmeal } \\
\text { Potassium carbonate, at the plant } \\
\text { (Europe) } \\
\text { Sulfur (elemental) at the refinery } \\
\text { (US) } \\
\text { Yeast (surrogate data, yeast } \\
\text { produced as a co-product) } \\
\text { Serenade is a strain of Bacillus subtilis } \\
\text { (Swiss) } \\
\text { Glyphosate, at regional storehouse } \\
\text { (Europe) } \\
\text { Diphenyl-ether compounds at } \\
\text { regional storehouse (Europe) } \\
\text { Phtalamide compounds at regional } \\
\text { storehouse (Europe) } \\
\text { Pesticide unspecified, at regional } \\
\text { storehouse RER } \\
\text { Developed based on Recycled } \\
\text { Organics Unit (2006), } \\
\text { updated with regionally appropriate } \\
\text { LCI datasets } \\
\text { Electricity grid mix (West US) } \\
\text { Modeled based on power rating and } \\
\text { hours of operation } \\
\text { (California model) and Diesel (US) } \\
\text { Truck (combination)—diesel } \\
\text { rail (US diesel) }\end{array}$ & $\begin{array}{l}\text { Gasoline } \\
\text { Diesel } \\
\text { Urea ammonium nitrate (UAN) } \\
\text { Monoammonium phosphate (MAP) } \\
\text { Adjuvant (stylet oil) } \\
\text { Potassium sulfate } \\
\text { Phytamin component: seabird } \\
\text { guano } \\
\text { Phytamin component: fishmeal } \\
\text { Phytamin component: potassium } \\
\text { carbonate } \\
\text { Sulfur dust } \\
\text { Serenade } \\
\text { Roundup Ultra Max } \\
\text { Goal 2XL } \\
\text { Chateau, Pristine (Boscalid and } \\
\text { Pyraclostrobin) } \\
\text { Compost production } \\
\text { Electricity } \\
\text { Equipment operation } \\
\text { Truck, rail shipping } \\
\text { International shipping }\end{array}$ & NA & $\begin{array}{l}\text { IPCC Tier } 2 \text { emissions were used to } \\
\text { calculate the field-based } \\
\mathrm{N}_{2} \mathrm{O} \text { emissions from fertilizer and } \\
\text { compost application and } \\
\text { vineyard plant matter, including } \\
\text { leaves, clippings, and cover } \\
\text { crop residue following mowing } \\
\text { (Intergovernmental Panel on } \\
\text { Climate Change 2006; Point et al. } \\
\text { 2012). }\end{array}$ & NA \\
\hline
\end{tabular}


Table A2. Cont.

\begin{tabular}{|c|c|c|c|c|c|c|c|}
\hline$\#$ & Reference & Data Source & Practice & Input & Unit & Output & Unit \\
\hline 46 & [63] & $\begin{array}{l}\text { Secondary data } \\
\text { (databases) }\end{array}$ & $\begin{array}{l}\text { Fossil energy life cycle factors for } \\
\text { agricultural inputs }\end{array}$ & $\begin{array}{l}\text { Nitrogen } \\
\text { Phosphorous } \\
\text { Potassium } \\
\text { Lime } \\
\text { Sulfur } \\
\text { Micronutrients } \\
\text { Cover crop seed } \\
\text { Herbicide } \\
\text { Insecticide } \\
\text { Fungicide } \\
\text { Gasoline } \\
\text { Diesel } \\
\text { Plastic } \\
\text { Agriculture machinery Electricity }\end{array}$ & $\begin{array}{l}\mathrm{MJ} / \mathrm{kg} \\
\mathrm{MJ} / \mathrm{kg} \\
\mathrm{MJ} / \mathrm{kg} \\
\mathrm{MJ} / \mathrm{kg} \\
\mathrm{MJ} / \mathrm{kg} \\
\mathrm{MJ} / \mathrm{kg} \\
\mathrm{MJ} / \mathrm{kg} \\
\mathrm{MJ} / \mathrm{kg} \\
\mathrm{MJ} / \mathrm{kg} \\
\mathrm{MJ} / \mathrm{kg} \\
\mathrm{MJ} / \mathrm{L} \\
\mathrm{MJ} / \mathrm{L} \\
\mathrm{MJ} / \mathrm{kg} \\
\mathrm{MJ} / \mathrm{h} \\
\mathrm{MJ} / \mathrm{kWh}\end{array}$ & $\begin{array}{l}\text { Direct } \mathrm{N}_{2} \mathrm{O} \text { emissions from } \\
\text { agricultural } \\
\text { Emissions (e.g., volatile organic } \\
\text { compound } \\
\text { (VOC), carbon monoxide }(\mathrm{CO}) \text {, } \\
\text { carbon dioxide }\left(\mathrm{CO}_{2}\right) \text {, nitrogen } \\
\text { monoxide }(\mathrm{NO}) \text {, nitrogen dioxide } \\
\left(\mathrm{NO}_{2}\right) \text {, nitrous oxide }\left(\mathrm{N}_{2} \mathrm{O}\right) \text {, } \\
\text { particulate matter }(\mathrm{PM} 10) \text {, } \\
\text { particulate matter (PM2.5), sulfur } \\
\text { dioxide }\left(\mathrm{SO}_{2}\right) \text {, sulfur trioxide }\left(\mathrm{SO}_{3}\right) \text {, } \\
\text { methane }\left(\mathrm{CH}_{4}\right) \text { ) } \\
\text { Emissions and energy use in } \\
\text { transportation }\end{array}$ & NA \\
\hline 47 & [24] & $\begin{array}{l}\text { Secondary data } \\
\text { (databases) }\end{array}$ & $\begin{array}{l}\text { Electricity production } \\
\text { Oil production Plastic P1 } \\
\text { production } \\
\text { Gutter A1 production } \\
\text { Gutter A1 use and demolition } \\
\text { Incineration of P1/A1 } \\
\text { Recycling process Material B } \\
\text { production } \\
\text { Product system } \\
\text { Electricity production } \\
\text { Oil production Plastic P2 } \\
\text { production } \\
\text { Gutter A2 production } \\
\text { Gutter A2 use and demolition } \\
\text { Incineration of P2/A2 } \\
\text { Recycling process Material B } \\
\text { production } \\
\text { Product system }\end{array}$ & $\begin{array}{l}\text { Electricity } \\
\text { Oil } \\
\text { Plastic P1 } \\
\text { Produced A1 } \\
\text { Installed A1 } \\
\text { Incinerated P1/A } \\
\text { A1 in recycling } \\
\text { Avoided material B }\end{array}$ & $\begin{array}{l}\text { MJ } \\
\mathrm{kg} \\
\mathrm{kg} \\
100 \mathrm{~m} \\
100 \mathrm{~m} \\
\mathrm{~kg} \\
\mathrm{~kg} \\
\mathrm{~kg}\end{array}$ & $\begin{array}{l}\mathrm{CO}_{2} \\
\mathrm{CH}_{4} \\
\mathrm{~N}_{2} \mathrm{O} \\
\mathrm{NO}_{\mathrm{x}} \\
\mathrm{SO}_{2}\end{array}$ & $\begin{array}{l}\mathrm{kg} \\
\mathrm{kg} \\
\mathrm{kg} \\
\mathrm{kg} \\
\mathrm{kg}\end{array}$ \\
\hline
\end{tabular}


Table A2. Cont.

\begin{tabular}{|c|c|c|c|c|c|c|c|}
\hline$\#$ & Reference & Data Source & Practice & Input & Unit & Output & Unit \\
\hline 48 & [64] & $\begin{array}{l}\text { Secondary data } \\
\text { (databases) }\end{array}$ & $\begin{array}{l}\text { Planting and maintenance } \\
\text { Harvesting and baling } \\
\text { Receiving/storage } \\
\text { Drying and chopping } \\
\text { Pelletizing/cooling/screening } \\
\text { Packing and storage }\end{array}$ & $\begin{array}{l}\text { Seed } \\
\text { Fertilizer } \\
\text { Pesticide/herbicide } \\
\text { Land use } \\
\text { Machinery } \\
\text { Fuel } \\
\text { Machinery } \\
\text { Fuel } \\
\text { Electricity } \\
\text { Air } \\
\text { Plastic bag }\end{array}$ & $\begin{array}{l}\mathrm{kg} \cdot \mathrm{ha}^{-1} \\
\mathrm{ha} \\
\mathrm{kg} \cdot \mathrm{ha}^{-1} \\
\mathrm{ha} \\
\text { MJ } \\
\text { MJ } \\
\mathrm{kWh}\end{array}$ & $\begin{array}{l}\text { Strawbale } \\
\mathrm{CO}_{2} \\
\mathrm{~N}_{2} \mathrm{O} \\
\mathrm{CH}_{4} \\
\mathrm{SO}_{2} \\
\mathrm{PO}_{4} \\
\text { Pellet }\end{array}$ & $\begin{array}{l}\mathrm{kg} \\
\mathrm{g} \mathrm{CO}_{2} \text { eq } \\
\mathrm{g} \mathrm{CO}_{2} \text { eq } \\
\mathrm{g} \mathrm{CO}_{2} \text { eq } \\
\mathrm{g} \mathrm{CO}_{2} \text { eq } \\
\mathrm{g} \mathrm{SO}_{2} \text { eq } \\
\mathrm{g} \mathrm{PO}_{4} \text { eq } \\
\mathrm{kg}\end{array}$ \\
\hline 49 & [65] & $\begin{array}{l}\text { Primary data } \\
\text { (real farm) } \\
\text { Secondary data } \\
\text { (database and previous } \\
\text { studies) }\end{array}$ & $\begin{array}{l}\text { Production characteristics } \\
\text { Greenhouse plastic } \\
\text { Water consumption } \\
\text { Growing media } \\
\text { Fertilizer } \\
\text { Pesticide } \\
\text { Electric power } \\
\text { Diesel and petrol } \\
\text { Post-harvest chemicals }\end{array}$ & $\begin{array}{l}\text { Plastic consumption } \\
\text { Rejected steams } \\
\text { Power consumption } \\
\text { Diesel } \\
\text { Petrol } \\
\text { Cardboard box } \\
\text { Bunching paper } \\
\text { Rubber band } \\
\text { Strapping roll } \\
\text { Water } \\
\text { Substrate (red ash) } \\
\text { Pesticide } \\
\text { Pesticide empty containers } \\
\text { Calcium nitrate } \\
\text { Other fertilizers } \\
\text { Acids } \\
\text { Post-harvest chemicals } \\
\text { Post-harvest water use }\end{array}$ & $\begin{array}{l}\mathrm{g} \\
\# \\
\mathrm{kWh} \\
\mathrm{g} \\
\mathrm{g} \\
\mathrm{g} \\
\mathrm{g} \\
\mathrm{g} \\
\mathrm{g} \\
\mathrm{L} \\
\mathrm{g} \\
\mathrm{g} \\
\mathrm{g} \\
\mathrm{g} \\
\mathrm{g} \\
\mathrm{g} \\
\mathrm{g} \\
\mathrm{L}\end{array}$ & $\begin{array}{l}\text { Roses } \\
\mathrm{CO}_{2} \\
\mathrm{CH}_{4} \\
\mathrm{~N}_{2} \mathrm{O}\end{array}$ & $\begin{array}{l}\text { Bunch } \\
\text { g } \\
\mathrm{g} \\
\mathrm{g}\end{array}$ \\
\hline
\end{tabular}


Table A2. Cont.

\begin{tabular}{|c|c|c|c|c|c|c|c|}
\hline \# & Reference & Data Source & Practice & Input & Unit & Output & Unit \\
\hline 50 & [32] & $\begin{array}{l}\text { Secondary data } \\
\text { (database and previous } \\
\text { studies) }\end{array}$ & $\begin{array}{l}\text { Production of crop inputs, } \\
\text { production and use of diesel, and } \\
\text { field emissions }\end{array}$ & $\begin{array}{l}\mathrm{N} \text { (ammonium nitrate) } \\
\mathrm{P}_{2} \mathrm{O}_{5} \text { (triple superphosphate } \\
\mathrm{K}_{2} \mathrm{O} \text { (potassium chloride) } \\
\mathrm{CaO} \\
\text { Seed for sowing } \\
\text { Pesticide (active ingredient) } \\
\text { Diesel } \\
\text { Natural gas (for grain drying) } \\
\text { Agricultural machinery } \\
\text { Grain dry matter yield } \\
\text { Stem/straw dry matter yield } \\
\text { Sugar/tuber dry matter yield } \\
\text { Followed bycatch crop }(\%) \\
\text { Succeeding crop } \mathrm{NO}_{3}-\mathrm{N} \text { emitted }\end{array}$ & $\begin{array}{l}\mathrm{kg} / \mathrm{ha} \\
\mathrm{kg} / \mathrm{ha} \\
\mathrm{kg} / \mathrm{ha} \\
\mathrm{kg} / \mathrm{ha} \\
\mathrm{kg} / \mathrm{ha} \\
\mathrm{kg} / \mathrm{ha} \\
\mathrm{kg} / \mathrm{ha} \\
\mathrm{kg} / \mathrm{ha} \\
\mathrm{kg} / \mathrm{ha} \\
\mathrm{kg} / \mathrm{ha} \\
\mathrm{kg} / \mathrm{ha} \\
\mathrm{kg} / \mathrm{ha} \\
\mathrm{kg} / \mathrm{ha}\end{array}$ & $\begin{array}{l}\text { Hemp } \\
\text { Sunflower } \\
\text { Rapeseed } \\
\text { Pea } \\
\text { Wheat } \\
\text { Maize } \\
\text { Potato } \\
\text { Sugar beet } \\
\mathrm{NH}_{3}-\mathrm{N} \\
\mathrm{NO}_{3}-\mathrm{N} \\
\mathrm{N}_{2} \mathrm{O}-\mathrm{N} \\
\mathrm{PO}_{4}-\mathrm{P}\end{array}$ & $\begin{array}{l}\text { ha } \\
\text { ha } \\
\text { ha } \\
\text { ha } \\
\text { ha } \\
\text { ha } \\
\text { ha } \\
\text { ha } \\
\text { emissions } / \mathrm{kg} \\
\text { emissions } / \mathrm{kg} \\
\text { emissions } / \mathrm{kg} \\
\text { emissions } / \mathrm{kg}\end{array}$ \\
\hline 51 & [100] & $\begin{array}{l}\text { Secondary data } \\
\text { (database and previous } \\
\text { studies) }\end{array}$ & $\begin{array}{l}\text { Infrastructure: } \\
\text { - } \quad \text { Buildings } \\
\text { Fieldwork processes: } \\
\text { - } \quad \text { Soil cultivation } \\
\text { - } \quad \text { Fertilization } \\
\text { - } \quad \text { Cheming } \\
\text { - } \quad \text { Mechanical plant protection treatment } \\
\text { - } \\
\text { Harvest } \\
\text { Transport }\end{array}$ & $\begin{array}{l}\text { Mineral fertilizers } \\
\text { Organic fertilizers } \\
\text { Pesticides } \\
\text { Seed } \\
\text { Feed }\end{array}$ & $\begin{array}{l}\mathrm{kg} \\
\mathrm{kg} \\
\mathrm{kg} \\
\mathrm{kg} \\
\mathrm{kg}\end{array}$ & $\begin{array}{l}\text { Potatoes organic, at the farm } \\
\text { Rapeseed extensive, at the farm } \\
\text { Wheat grains conventional, Barrois, } \\
\text { at the farm } \\
\text { Carbon dioxide } \mathrm{CO}_{2} \\
\text { Sulfur dioxide } \mathrm{SO}_{2} \\
\text { Lead Pb } \\
\text { Methane } \mathrm{CH}_{4} \\
\text { Benzene } \mathrm{C}_{6} \mathrm{H}_{6} \\
\text { Particulate Matter PM } \\
\text { Cadmium Cd } \\
\text { Chromium } \mathrm{Cr} \\
\text { Copper } \mathrm{Cu} \\
\text { Monoxide } \mathrm{N}_{2} \mathrm{O} \\
\text { Nickel } \mathrm{Ni}\end{array}$ & $\begin{array}{l}\mathrm{kg} \\
\mathrm{kg} \\
\mathrm{kg} \\
\mathrm{g} / \mathrm{kg} \\
\mathrm{g} / \mathrm{kg} \\
\mathrm{g} / \mathrm{kg} \\
\mathrm{g} / \mathrm{kg} \\
\mathrm{g} / \mathrm{kg} \\
\mathrm{g} / \mathrm{kg} \\
\mathrm{g} / \mathrm{kg} \\
\mathrm{g} / \mathrm{kg} \\
\mathrm{g} / \mathrm{kg} \\
\mathrm{g} / \mathrm{kg}\end{array}$ \\
\hline
\end{tabular}


Table A2. Cont.

\begin{tabular}{|c|c|c|c|c|c|c|c|}
\hline$\#$ & Reference & Data Source & Practice & Input & Unit & Output & Unit \\
\hline 52 & [85] & Primary data (survey) & $\begin{array}{l}\text { Tractors and equipment } \\
\text { Buildings required energy } \\
\text { Carriers } \\
\text { Mineral fertilizer } \\
\text { Tree nursing } \\
\text { Constructions for hail protection } \\
\text { Water for irrigation } \\
\text { Application of compost }\end{array}$ & $\begin{array}{l}\text { Pesticides } \\
\text { Fungicide } \\
\text { Insecticide } \\
\text { Herbicide } \\
\text { Other plants } \\
\text { treatment products } \\
\text { Fertilizers } \\
\mathrm{N} \text {-fertilizer } \\
\text { Ca- and Mg-fertilizer } \\
\text { (kg Ca, Mg) } \\
\text { K-fertilizer } \\
\text { P-fertilizer } \\
\text { Machinery } \\
\text { Diesel } \\
\text { Tractor } \\
\text { Equipment } \\
\text { Buildings }\end{array}$ & $\begin{array}{l}\mathrm{kg} \text { active matter } \\
\mathrm{kg} \mathrm{N} \\
\mathrm{kg} \mathrm{K}_{2} \mathrm{O} \\
\mathrm{kg} \mathrm{P}_{2} \mathrm{O}_{5} \\
\mathrm{~kg} \\
\mathrm{~kg} \\
\mathrm{~kg} \\
\mathrm{~m}^{2}\end{array}$ & $\begin{array}{l}\text { Total receipts } \\
\text { Yield }\end{array}$ & $\begin{array}{l}\text { USD.ha }{ }^{-1} \\
\mathrm{t} \cdot \mathrm{ha}^{-1}\end{array}$ \\
\hline 53 & [89] & $\begin{array}{l}\text { Secondary data } \\
\text { (databases) }\end{array}$ & $\begin{array}{l}\text { Pesticide } \\
\text { Seeds } \\
\text { PK fertilizer } \\
\mathrm{N} \text { fertilizer } \\
\text { Machinery mulching } \\
\text { Machinery irrigation } \\
\text { Machinery pesticide } \\
\text { Machinery fertilization } \\
\text { Machinery weeding } \\
\text { Machinery soil tillage } \\
\text { Machinery harvest } \\
\text { Machinery sowing }\end{array}$ & Energy input & MJ eq & $\begin{array}{l}\mathrm{CH}_{4} \\
\mathrm{~N}_{2} \mathrm{O} \\
\mathrm{CO}_{2} \\
\mathrm{Ph} \\
\mathrm{NH}_{3} \\
\mathrm{NO}^{-3}\end{array}$ & $\begin{array}{l}\mathrm{t} \mathrm{CO}_{2} \\
\text { eq } \cdot \mathrm{ha}^{-1} \cdot \mathrm{year}^{-1} \\
\mathrm{~kg} \mathrm{~N} \\
\text { eq } \mathrm{ha}^{-1} \cdot \mathrm{year}^{-1} \\
\mathrm{t} \mathrm{CO}_{2} \\
\text { eq } \cdot \mathrm{ha}^{-1} \cdot \mathrm{year}^{-1} \\
\mathrm{~kg} \mathrm{~N} \\
\text { eq } \mathrm{ha}^{-1} \cdot \mathrm{year}^{-1} \\
\mathrm{~kg} \mathrm{~N} \\
\text { eq } \cdot \mathrm{ha}^{-1} \cdot \mathrm{year}^{-1} \\
\mathrm{~kg} \mathrm{~N} \\
\text { eq } \cdot \mathrm{ha}^{-1} \cdot \mathrm{year}^{-1}\end{array}$ \\
\hline
\end{tabular}


Table A2. Cont.

Grape

Ammonia $\left(\mathrm{NH}_{3}\right)$

Ammonia $\left(\mathrm{NH}_{3}\right)$

Benzen

Benzo (a) pyrene

Cadmium (Cd)

Carbon dioxide $\left(\mathrm{CO}_{2}\right)$

$\mathrm{kg} \cdot \mathrm{ha}^{-1}$

$\mathrm{kg} \cdot \mathrm{ha}^{-1}$

Inputs

Carbon dioxide $\left(\mathrm{CO}_{2}\right)$ from urea.

Carbon monoxide $(\mathrm{CO})$

Chromium $(\mathrm{Cr})$

1. Diesel fuel

3. Human labor

Copper $(\mathrm{Cu})$

. Chemical fertilizers

Primary data (farmers) Transportation

(a) Nitrogen

b) Phosphate

Secondary data Fertilization

c) Potassium

(d) Sulfur

(databases and Pesticides

5. Manure

6. Chemical pesticides

(a) Fungicide

Dinitroger

Dinitrogen monoxide $\left(\mathrm{N}_{2} \mathrm{O}\right)$

Dinitrogen monoxide $\left(\mathrm{N}_{2} \mathrm{O}\right)$

Dinitrogen monoxide $\left(\mathrm{N}_{2} \mathrm{O}\right)$ from

atmospheric deposition

Hydrocarbons (HC, as NMVOC)

Methane $\left(\mathrm{CH}_{4}\right)$

(b) Insecticid

Nickel (Ni)

7. Irrigation

Nitrate $\left(\mathrm{NO}_{3}\right)$

Nitrogen oxide $\left(\mathrm{NO}_{\mathrm{x}}\right)$

Nitrogen oxides $\left(\mathrm{NO}_{\mathrm{x}}\right)$

PAH (polycyclic hydrocarbons)

The total energy input

Particulates (b2.5 mm)

Phosphorus emissions from

groundwater.

Selenium (Se)

Sulfur dioxide $\left(\mathrm{SO}_{2}\right)$

kg.ha ${ }^{-1}$

$\mathrm{kg} \cdot \mathrm{ha}^{-1}$

$\mathrm{kg} \cdot \mathrm{ha}^{-1}$

$\mathrm{kg} \cdot \mathrm{ha}^{-1}$

$\mathrm{kg} \cdot \mathrm{ha}^{-1}$

$\mathrm{kg} \cdot \mathrm{ha}^{-1}$

kg.ha ${ }^{-1}$

$\mathrm{kg} \cdot \mathrm{ha}^{-1}$

kg.ha ${ }^{-1}$

kg.ha ${ }^{-1}$

$\mathrm{kg} \cdot \mathrm{ha}^{-1}$

kg.ha

$\mathrm{kg} \cdot \mathrm{ha}^{-1}$

$\mathrm{kg} \cdot \mathrm{ha}^{-1}$

$\mathrm{kg} \cdot \mathrm{ha}^{-1}$

$\mathrm{kg} \cdot \mathrm{ha}^{-1}$

$\mathrm{kg} \cdot \mathrm{ha}^{-1}$

$\mathrm{kg} \cdot \mathrm{ha}^{-1}$

$\mathrm{kg} \cdot \mathrm{ha}^{-1}$

$\mathrm{kg} \cdot \mathrm{ha}^{-1}$

$\mathrm{kg} \cdot \mathrm{ha}^{-1}$

$\mathrm{kg} \cdot \mathrm{ha}^{-1}$ 
Table A2. Cont.

\begin{tabular}{|c|c|c|c|c|c|c|c|}
\hline$\#$ & Reference & Data Source & Practice & Input & Unit & Output & Unit \\
\hline 55 & [83] & $\begin{array}{l}\text { Primary data } \\
\text { (field experiment) } \\
\text { Secondary data } \\
\text { (database) }\end{array}$ & $\begin{array}{l}\text { Fertilization } \\
\text { Cutting preparation } \\
\text { Spraying } \\
\text { Ploughing } \\
\text { Disking } \\
\text { Harrowing } \\
\text { Marking } \\
\text { Spraying } \\
\text { Mechanical weeding } \\
\text { Fertilizing } \\
\text { Lignin production } \\
\text { and application } \\
\text { Harvest } \\
\text { Transport } \\
\text { Liquidation }\end{array}$ & $\begin{array}{l}\text { Tractor/harvester } \\
\text { Machinery } \\
\text { Diesel fuel }\end{array}$ & $\mathrm{kg} \cdot \mathrm{ha}^{-1}$ & $\begin{array}{l}\mathrm{CO}_{2} \\
\mathrm{PM} \\
\mathrm{SO}_{2} \\
\mathrm{p}\end{array}$ & $\begin{array}{l}\mathrm{kg} \mathrm{Mg}^{-1} \mathrm{CO}_{2} \text { eq } \\
\mathrm{kg} \mathrm{MP} 10 \text { eq } \mathrm{kg} \mathrm{SO}_{2} \text { eq } \\
\mathrm{kg} \mathrm{p} \mathrm{eq}\end{array}$ \\
\hline 56 & [90] & $\begin{array}{l}\text { Secondary data } \\
\text { (databases and previous } \\
\text { studies) }\end{array}$ & Pesticide application & Active ingredients of the pesticide & $\mathrm{t}$ & Active ingredients emissions & Unitless \\
\hline 57 & [66] & $\begin{array}{l}\text { Primary } \\
\text { (survey) } \\
\text { Secondary data } \\
\text { (database and previous } \\
\text { studies) }\end{array}$ & $\begin{array}{l}\text { Nursery } \\
\text { Tomato cultivation } \\
\text { Packaging } \\
\text { Transportation }\end{array}$ & $\begin{array}{l}\text { Reporting period } \\
\text { Country } \\
\text { (Production site) } \\
\text { Growing period } \\
\text { Greenhouse } \\
\text { structure } \\
\text { Substrate } \\
\text { Greenhouse } \\
\text { heating } \\
\mathrm{CO}_{2} \text { enrichment } \\
\text { Yield } \\
\text { Fertilization } \\
\text { Irrigation } \\
\text { Energy } \\
\text { consumption }\end{array}$ & $\begin{array}{l}\text { ton } \cdot \mathrm{ha}^{-1} \\
\mathrm{~kg} \mathrm{~N} \cdot \mathrm{ha}^{-1}, \mathrm{~kg} \\
\mathrm{P}_{2} \mathrm{O}_{5} \cdot \mathrm{ha}^{-1}, \mathrm{~kg} \\
\mathrm{~K}_{2} \mathrm{O} \cdot \mathrm{ha}^{-1} \\
\text { water m }{ }^{3} \cdot \mathrm{ha}^{-1} \\
\mathrm{kWh} \cdot \mathrm{ha}^{-1}\end{array}$ & $\begin{array}{l}\text { Nitrogen oxides, phosphates, and } \\
\text { pesticides emissions } \\
\text { nitrous oxide, and ammonia }\end{array}$ & $\begin{array}{l}\text { g N eq } \\
\text { g P eq }\end{array}$ \\
\hline
\end{tabular}


Table A2. Cont.

\begin{tabular}{|c|c|c|c|c|c|c|c|}
\hline$\#$ & Reference & Data Source & Practice & Input & Unit & Output & Unit \\
\hline 58 & [86] & $\begin{array}{l}\text { Secondary data } \\
\text { (databases) }\end{array}$ & $\begin{array}{l}\text { Applying farmyard manure } \\
\text { Land preparation } \\
\text { Planting } \\
\text { Fertilizing } \\
\text { Harvesting }\end{array}$ & $\begin{array}{l}\text { N-based fertilizers } \\
\text { P-based fertilizers } \\
\text { K-based fertilizers } \\
\text { Pesticides } \\
\text { Farmyard manure } \\
\text { Microelements } \\
\text { Diesel fuel } \\
\text { Water }\end{array}$ & $\begin{array}{l}\mathrm{kg} \\
\mathrm{kg} \\
\mathrm{kg} \\
\mathrm{kg} \\
\mathrm{t} \\
\mathrm{kg} \\
\mathrm{L} \\
\mathrm{m}^{3}\end{array}$ & $\begin{array}{l}\text { Carbon dioxide }\left(\mathrm{CO}_{2}\right) \\
\text { Sulfur dioxide }\left(\mathrm{SO}_{2}\right) \\
\text { Methane }\left(\mathrm{CH}_{4}\right) \\
\text { Benzene } \\
\text { Cadmium }(\mathrm{Cd}) \\
\text { Chromium }(\mathrm{Cr}) \\
\text { Copper }(\mathrm{Cu}) \\
\text { Dinitrogen monoxide }\left(\mathrm{N}_{2} \mathrm{O}\right) \\
\text { Nickel }(\mathrm{Ni}) \\
\text { Zinc }(\mathrm{Zn}) \\
\text { Benzo(a)pyrene } \\
\text { Ammonia }\left(\mathrm{NH}_{3}\right) \\
\text { Selenium }(\mathrm{Se}) \\
\text { PAH (polycyclic hydrocarbons) } \\
\text { Hydrocarbons }(\mathrm{HC}, \text { as NMVOC) } \\
\left.\text { Nitrogen oxides }(\mathrm{NO})_{\times}\right) \\
\text {Carbon monoxide }(\mathrm{CO}) \\
\text { Particulates }(<2.5 \mu \mathrm{m})\end{array}$ & $\begin{array}{l}\mathrm{kg} \\
\mathrm{kg} \\
\mathrm{kg} \\
\mathrm{kg} \\
\mathrm{kg} \\
\mathrm{kg} \\
\mathrm{kg} \\
\mathrm{kg} \\
\mathrm{kg} \\
\mathrm{kg} \\
\mathrm{kg} \\
\mathrm{kg}\end{array}$ \\
\hline 59 & [67] & $\begin{array}{l}\text { Secondary data } \\
\text { (databases) }\end{array}$ & $\begin{array}{l}\text { Fertilization, } \\
\text { split fertilization, chemical fallow, } \\
\text { liming, } \\
\text { sowing and } \\
\text { spraying at the farm }\end{array}$ & $\begin{array}{l}\text { NPK-fertilizer } \\
\text { N-fertilizer } \\
\text { Roundup (glyphosate) } \\
\text { Dolomite (CaO) } \\
\text { Celest Formula M (fludioxonil) } \\
\text { Starane XL (fluroxypyr/florasulam) } \\
\text { Fastac } 50 \text { (alpha-ceypermethrin) }\end{array}$ & $\begin{array}{l}\mathrm{kg} \cdot \mathrm{ha}^{-1} \\
\mathrm{~kg} \cdot \mathrm{ha}^{-1} \\
\mathrm{~kg} \cdot \mathrm{ha}^{-1} \\
\mathrm{~kg} \cdot \mathrm{ha}^{-1} \\
\mathrm{~kg} \cdot \mathrm{ha}^{-1} \\
\mathrm{~kg} \cdot \mathrm{ha}^{-1} \\
\mathrm{~kg} \cdot \mathrm{ha}^{-1}\end{array}$ & $\begin{array}{l}\mathrm{N}_{2} \mathrm{O} \\
\mathrm{NH}_{3} \\
\mathrm{NO}_{\mathrm{x}}\end{array}$ & $\begin{array}{l}\mathrm{kg} \mathrm{N}_{2} \mathrm{O}-\mathrm{N} / \mathrm{kg} \mathrm{N} \\
\text { input } \\
\mathrm{kg} \mathrm{NH}_{3}-\mathrm{N} \\
\mathrm{NO}_{x}-\mathrm{N} / \mathrm{kg}\end{array}$ \\
\hline 60 & [103] & $\begin{array}{l}\text { Primary data } \\
\text { (real farm) } \\
\text { Secondary data } \\
\text { (databases) }\end{array}$ & $\begin{array}{l}\text { production, transport to } \\
\text { the farm and use on the farm }\end{array}$ & $\begin{array}{l}\text { Fertilizers, pesticides, } \\
\text { field materials, pesticide spray } \\
\text { equipment, irrigation system and } \\
\text { packaging manufacturing }\end{array}$ & NA & Pesticide emission & NA \\
\hline
\end{tabular}


Table A2. Cont.

\begin{tabular}{|c|c|c|c|c|c|c|c|}
\hline$\#$ & Reference & Data Source & Practice & Input & Unit & Output & Unit \\
\hline \multirow[t]{2}{*}{61} & \multirow[t]{2}{*}{ [87] } & \multirow[t]{2}{*}{$\begin{array}{l}\text { Secondary data } \\
\text { (databases and previous } \\
\text { studies) }\end{array}$} & 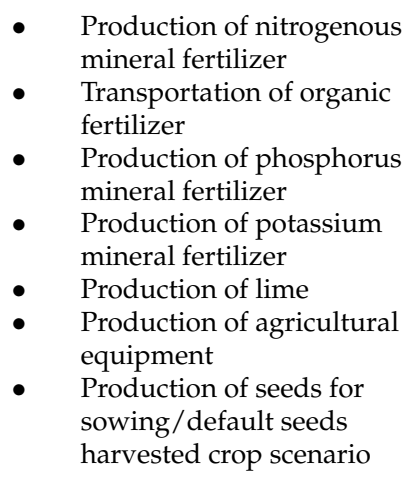 & $\begin{array}{l}\text { Fertilizer N, P, K } \\
\text { Lime } \\
\text { Fuel } \\
\text { Seeds } \\
\text { Agricultural } \\
\text { equipment }\end{array}$ & NA & $\begin{array}{l}\text { Agricultural engine } \\
\text { emissions }\left(\mathrm{CO}, \mathrm{HC}, \mathrm{NO}_{\mathrm{x}}\right. \\
\left.\mathrm{SO}_{2}, \mathrm{PM}, \mathrm{CO}_{2}\right)(\mathrm{EPA} \\
2004) \\
\text { Direct field emission from } \\
\text { fertilization }\left(\mathrm{NO}_{3}, \mathrm{NH}_{4},\right. \\
\left.\mathrm{N}_{2} \mathrm{O}, \mathrm{NO}_{x}, \mathrm{CO}_{2}, \mathrm{PO}_{4}\right) \\
\text { Hemp straws and seeds }\end{array}$ & NA \\
\hline & & & Production of diesel & & & & \\
\hline 62 & [91] & $\begin{array}{l}\text { Secondary data } \\
\text { (databases) }\end{array}$ & Pesticide application & $\begin{array}{l}\text { S-Metolachlor }(\mathrm{H}) \\
\text { Simazine }(\mathrm{H}) \\
\text { Glyphosate }(\mathrm{H}) \\
\text { Glufosinate ammonium }(\mathrm{H}) \\
\text { Dimethenamid-P (H) } \\
\text { Atrazine (H) } \\
\text { Alachlor (H) } \\
\text { Acetochlor (H) } \\
\text { 2,4-D-dimethylammonium .. } \\
\text { 2,4-D-2-ethylhexyl ester (H) } \\
\text { Fipronil (I) } \\
\text { Chlorpyrifos (I) }\end{array}$ & $\begin{array}{l}\mathrm{kg} / \mathrm{kg} \text { corn } \\
\mathrm{kg} / \mathrm{kg} \text { corn } \\
\mathrm{kg} / \mathrm{kg} \text { corn } \\
\mathrm{kg} / \mathrm{kg} \text { corn } \\
\mathrm{kg} / \mathrm{kg} \text { corn } \\
\mathrm{kg} / \mathrm{kg} \text { corn } \\
\mathrm{kg} / \mathrm{kg} \text { corn } \\
\mathrm{kg} / \mathrm{kg} \text { corn } \\
\mathrm{kg} / \mathrm{kg} \text { corn } \\
\mathrm{kg} / \mathrm{kg} \text { corn }\end{array}$ & $\begin{array}{l}\text { Pesticide emission to air, surface } \\
\text { water, and groundwater }\end{array}$ & $\%$ \\
\hline
\end{tabular}


Table A2. Cont.

\begin{tabular}{|c|c|c|c|c|c|c|c|}
\hline \# & Reference & Data Source & Practice & Input & Unit & Output & Unit \\
\hline 63 & [101] & $\begin{array}{l}\text { Primary data } \\
\text { (real farm) } \\
\text { Secondary data } \\
\text { (databases) }\end{array}$ & $\begin{array}{l}\text { Manufacture } \\
\text { of greenhouse components, } \\
\text { substrate, fertilizers, and pesticides. } \\
\text { the electricity production mix; and } \\
\text { transport and disposal of } \\
\text { materials }\end{array}$ & $\begin{array}{l}\text { greenhouse components Water } \\
\text { consumption and fertilizer and } \\
\text { pesticide doses applied }\end{array}$ & $\begin{array}{l}\mathrm{kg}, \mathrm{m}^{2}, \mathrm{~m}^{3} \\
\mathrm{M}^{3} \\
\mathrm{~kg}, \mathrm{~L} \\
\mathrm{~kg}\end{array}$ & $\begin{array}{l}\mathrm{N}_{2} \mathrm{O} \\
\mathrm{NO}_{x} \\
\mathrm{NH}_{3} \\
\text { Azoxystrobin } \\
\text { Chlorothalonil } \\
\text { Clofentezine } \\
\text { Fenbutatin oxide } \\
\text { Mancozeb } \\
\text { Spinosad } \\
\text { Copper chloride oxide, hydrate } \\
\text { Concrete } \\
\text { Plastics } \\
\text { Substrate }\end{array}$ & $\begin{array}{l}\mathrm{kg} \\
\mathrm{kg} \\
\mathrm{kg} \\
\mathrm{kg} \\
\mathrm{kg} \\
\mathrm{kg} \\
\mathrm{kg} \\
\mathrm{kg} \\
\mathrm{kg} \\
\mathrm{kg} \\
\mathrm{kg} \\
\mathrm{kg} \\
\mathrm{kg}\end{array}$ \\
\hline 64 & [94] & $\begin{array}{l}\text { Primary data (survey) } \\
\text { Secondary data } \\
\text { (databases and previous } \\
\text { studies) }\end{array}$ & 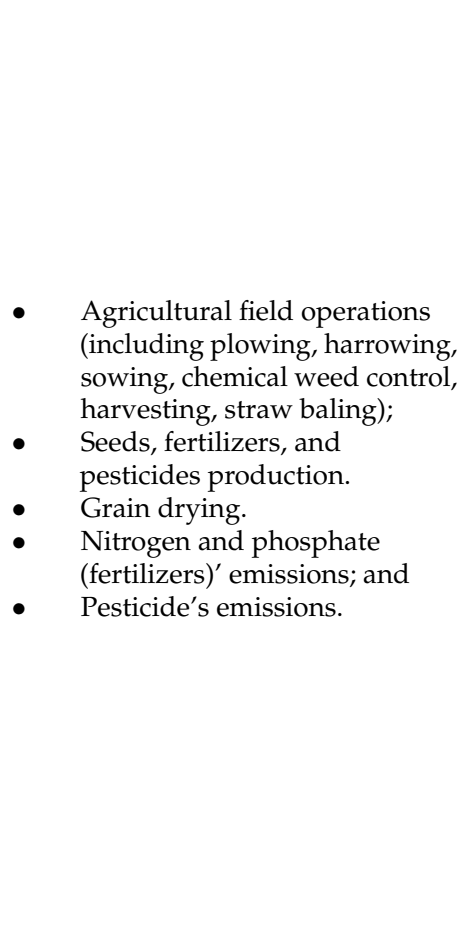 & $\begin{array}{l}\text { Yield } \\
\text { Grain } \\
\text { Straw } \\
\text { Agricultural field operations } \\
\text { Ploughing } \\
\text { Harrowing by rotary harrow } \\
\text { Sowing } \\
\text { Fertilizing by broadcaster } \\
\text { Slurry spreading } \\
\text { Pest control application by field } \\
\text { Sprayer } \\
\text { Harvesting } \\
\text { Bailing } \\
\text { Transport (tractor and trailer) } \\
\text { Grain drying } \\
\text { Seeds } \\
\text { Fertilizers } \\
\text { Calcium ammonium nitrate (CAN) } \\
\text { Ammonium nitrate } \\
\text { Pig slurry } \\
\text { Dairy cattle slurry } \\
\text { Pesticides } \\
\text { Tribenuron-methyl } \\
\text { Pyraclostrobin } \\
\text { Tebuconazole } \\
\text { Pirimicarb } \\
\text { Difensulfuron } \\
\text { 2,3-D-Bromoxinil }\end{array}$ & $\begin{array}{l}\mathrm{t} / \mathrm{ha} \\
\mathrm{t} / \mathrm{ha} \\
\text { number of } \\
\text { repetitions (rep) } \\
\text { rep } \\
\text { rep } \\
\text { rep } \\
\text { rep } \\
\text { rep } \\
\mathrm{rep} \\
\mathrm{rep} \\
\mathrm{rep} \\
\mathrm{rep} \\
\mathrm{rep} \\
\mathrm{kg} / \mathrm{ha} \\
\mathrm{kg} \mathrm{N} / \mathrm{ha} \\
\mathrm{kg} \mathrm{N} / \mathrm{ha} \\
\mathrm{kg} \mathrm{N} / \mathrm{ha} \\
\mathrm{kg} \mathrm{N} / \mathrm{ha} \\
\mathrm{g} / \mathrm{ha} \\
\mathrm{g} / \mathrm{ha} \\
\mathrm{g} / \mathrm{ha} \\
\mathrm{g} / \mathrm{ha} \\
\mathrm{g} / \mathrm{ha} \\
\mathrm{g} / \mathrm{ha} \\
\mathrm{g} / \mathrm{ha}\end{array}$ & $\begin{array}{l}\text { Fertilizers' emissions } \\
\mathrm{NH}_{3} \\
\mathrm{~N}_{2} \mathrm{O} \\
\mathrm{NO} 3 \\
\mathrm{PO} 4 \\
\text { Pesticides' emissions } \\
\text { Tribenuron-methyl } \\
\text { Pyraclostrobin } \\
\text { Tebuconazole } \\
\text { Pirimicarb } \\
\text { Thifensulfuron-methyl } \\
\text { (difensulfuron) } \\
\text { 2,4-D-Bromoxynil }\end{array}$ & $\begin{array}{l}\mathrm{kg} / \mathrm{ha} \\
\mathrm{kg} / \mathrm{ha} \\
\mathrm{kg} / \mathrm{ha} \\
\mathrm{kg} / \mathrm{ha} \\
\mathrm{kg} / \mathrm{ha} \\
\mathrm{kg} / \mathrm{ha} \\
\mathrm{kg} / \mathrm{ha} \\
\mathrm{kg} / \mathrm{ha} \\
\mathrm{kg} / \mathrm{ha} \\
\mathrm{kg} / \mathrm{ha} \\
\mathrm{kg} / \mathrm{ha}\end{array}$ \\
\hline
\end{tabular}


Table A2. Cont.

\begin{tabular}{|c|c|c|c|c|c|c|c|}
\hline$\#$ & Reference & Data Source & Practice & Input & Unit & Output & Unit \\
\hline 65 & {$[80]$} & $\begin{array}{l}\text { Secondary data } \\
\text { (databases) }\end{array}$ & $\begin{array}{l}\text { Slurry tanker } \\
\text { and spreading device production } \\
\text { Tractor production } \\
\text { Diesel production }\end{array}$ & $\begin{array}{l}\text { Raw materials } \\
\text { Energy }\end{array}$ & & $\begin{array}{l}\text { Field emissions } \mathrm{NH}_{3}, \mathrm{~N}_{2} \mathrm{O}, \mathrm{NO}_{3} \text {, } \\
\mathrm{PO}_{4} \\
\text { Other emissions } \\
\text { to air, soil, water }\end{array}$ & $\mathrm{kg} \mathrm{N}$ \\
\hline 66 & [84] & $\begin{array}{l}\text { Secondary data } \\
\text { (databases) }\end{array}$ & $\begin{array}{l}\text { Transportation of raw materials } \\
\text { Production of technical oxide } \\
\text { Transportation of technical oxide } \\
\text { Production of fertilizer } \\
\text { Transportation of fertilizer } \\
\text { Spreading }\end{array}$ & Zinc ashes & $\begin{array}{l}9 \mathrm{~kg} \cdot \mathrm{ha}^{-1} \\
\text { every three } \\
\text { years }\end{array}$ & $\begin{array}{l}\mathrm{Zn} \\
\mathrm{ZnCl}_{2} \\
\mathrm{ZnO}\end{array}$ & $\begin{array}{l}\mathrm{kg} \\
\mathrm{kg} \\
\mathrm{kg}\end{array}$ \\
\hline 67 & [68] & $\begin{array}{l}\text { Primary data } \\
\text { (interview) }\end{array}$ & $\begin{array}{l}\text { Desiccation } \\
\text { Liming } \\
\text { Soybean and sunflower seeds } \\
\text { treatment } \\
\text { Sowing and fertilization } \\
\text { Topdressing fertilization } \\
\text { Pesticide and herbicide application } \\
\text { Soybean and sunflower harvesting }\end{array}$ & $\begin{array}{l}\text { Product } \\
\text { Resources } \\
\text { Occupation, arable, } \\
\text { non-irrigated } \\
\text { Materials/fuels } \\
\text { Seeds } \\
\text { Limestone } \\
\text { Urea, as } \mathrm{N} \\
\text { Single superphosphate, } \\
\text { as } \mathrm{P}_{2} \mathrm{O}_{5} \\
\text { Triple superphosphate, } \\
\text { as } \mathrm{P}_{2} \mathrm{O}_{5} \\
\text { Potassium chloride, } \\
\text { as } \mathrm{K}_{2} \mathrm{O} \\
\text { Herbicides } \\
\text { Insecticides } \\
\text { Fungicides } \\
\text { Mineral oil } \\
\text { Boric acid } \\
\text { Liming } \\
\text { Pesticide application } \\
\text { Sowing and fertilization } \\
\text { Pesticide application } \\
\text { Harvesting }\end{array}$ & $\begin{array}{l}\mathrm{kg} \\
\mathrm{ha} \cdot \mathrm{year}^{-1} \\
\mathrm{~kg} \\
\mathrm{~kg} \\
\mathrm{~kg} \\
\mathrm{~kg} \\
\mathrm{~kg} \\
\mathrm{~kg} \\
\mathrm{~kg} \\
\mathrm{~kg} \\
\mathrm{~kg} \\
\mathrm{~kg} \\
\mathrm{~kg} \\
\mathrm{ha} \\
\mathrm{ha} \\
\mathrm{ha} \\
\mathrm{ha} \\
\mathrm{ha}\end{array}$ & $\begin{array}{l}\text { Emissions to air } \\
\text { Ammonia } \\
\text { Dinitrogen monoxide } \\
\text { Nitrogen oxides } \\
\mathrm{CO}_{2} \text {, fossil } \\
\mathrm{CO}_{2} \text {, land transformation } \\
\text { Emissions to water } \\
\text { Nitrate } \\
\text { Cadmium } 2 \\
\text { Copper } \\
\text { Zinc } \\
\text { Lead } \\
\text { Nickel } \\
\text { Chromium } \\
\text { Emissions to soil } \\
\text { Cadmium } \\
\text { Copper } \\
\text { Zinc } \\
\text { Lead } \\
\text { Nickel } \\
\text { Chromium } \\
\text { Herbicides } \\
\text { Insecticides } \\
\text { Fungicides }\end{array}$ & $\begin{array}{l}\mathrm{kg} \\
\mathrm{kg} \\
\mathrm{kg} \\
\mathrm{kg} \\
\mathrm{kg} \\
\mathrm{kg} \\
\mathrm{kg} \\
\mathrm{kg} \\
\mathrm{kg} \\
\mathrm{kg} \\
\mathrm{kg} \\
\mathrm{kg} \\
\mathrm{kg} \\
\mathrm{kg} \\
\mathrm{kg} \\
\mathrm{kg} \\
\mathrm{kg} \\
\mathrm{kg} \\
\mathrm{kg} \\
\mathrm{kg} \\
\mathrm{kg}\end{array}$ \\
\hline
\end{tabular}


Table A2. Cont.

\begin{tabular}{|c|c|c|c|c|c|c|c|}
\hline$\#$ & Reference & Data Source & Practice & Input & Unit & Output & Unit \\
\hline 68 & [99] & $\begin{array}{l}\text { Primary data } \\
\text { (survey) } \\
\text { Secondary data } \\
\text { (databases) }\end{array}$ & $\begin{array}{l}\text { Direct agricultural inputs } \\
\text { Production of the different } \\
\text { agricultural } \\
\text { inputs, }\end{array}$ & $\begin{array}{l}\text { Information about tractors and } \\
\text { implements, labor hours, and input } \\
\text { rates such as agrochemicals and } \\
\text { water use) } \\
\text { nitrogen (urea and ammonium } \\
\text { nitrate), } \\
\text { phosphorous or potassium-based } \\
\text { fertilizers and herbicides } \\
\text { (terbutilazine, } \\
\text { alachlor, lumax, and S-metolachlor }\end{array}$ & & $\begin{array}{l}\mathrm{NH}_{3} \\
\mathrm{~N}_{2} \mathrm{O} \\
\mathrm{NO}_{3}\end{array}$ & $\begin{array}{l}\mathrm{kg} \\
\mathrm{N}_{2} \mathrm{O}-\mathrm{N} \cdot \mathrm{ha}^{-1} \cdot \mathrm{kg}^{-1}\end{array}$ \\
\hline 69 & {$[92]$} & $\begin{array}{l}\text { Secondary data } \\
\text { (databases and previous } \\
\text { studies) }\end{array}$ & Pesticide application & $\begin{array}{l}\text { Abamectin } \\
\text { Azadirachtin } \\
\text { Chlorpyrifos } \\
\text { Clofentezine } \\
\text { Copper oxychloride } \\
\text { Fenazaquin } \\
\text { Fenbutatin-oxyde } \\
\text { Fluroxypyr } \\
\text { Fosetyl-Al } \\
\text { Glufosinate-ammonium } \\
\text { Glyphosate } \\
\text { Hexythiazox } \\
\text { Imazalil } \\
\text { Imidacloprid Insecticide } \\
\text { Lambda-cyhalothrin } \\
\text { Mancozeb } \\
\text { MCPA } \\
\text { Paraquat } \\
\text { Propargite } \\
\text { Pyridaben } \\
\text { Pyriproxyfen } \\
\text { Spinosad } \\
\text { Tebufenpyrad } \\
\text { Thiabendazole } \\
\text { White mineral oil (paraffin oil) }\end{array}$ & $\begin{array}{l}\mathrm{kg} \cdot \mathrm{m}^{-2} \\
\mathrm{~kg} \cdot \mathrm{m}^{-2} \\
\mathrm{~kg} \cdot \mathrm{m}^{-2} \\
\mathrm{~kg} \cdot \mathrm{m}^{-2} \\
\mathrm{~kg} \cdot \mathrm{m}^{-2} \\
\mathrm{~kg} \cdot \mathrm{m}^{-2} \\
\mathrm{~kg} \cdot \mathrm{m}^{-2} \\
\mathrm{~kg} \cdot \mathrm{m}^{-2} \\
\mathrm{~kg} \cdot \mathrm{m}^{-2} \\
\mathrm{~kg} \cdot \mathrm{m}^{-2} \\
\mathrm{~kg} \cdot \mathrm{m}^{-2} \\
\mathrm{~kg} \cdot \mathrm{m}^{-2} \\
\mathrm{~kg} \cdot \mathrm{m}^{-2} \\
\mathrm{~kg} \cdot \mathrm{m}^{-2} \\
\mathrm{~kg} \cdot \mathrm{m}^{-2} \\
\mathrm{~kg} \cdot \mathrm{m}^{-2} \\
\mathrm{~kg} \cdot \mathrm{m}^{-2} \\
\mathrm{~kg} \cdot \mathrm{m}^{-2} \\
\mathrm{~kg} \cdot \mathrm{m}^{-2} \\
\mathrm{~kg} \cdot \mathrm{m}^{-2} \\
\mathrm{~kg} \cdot \mathrm{m}^{-2} \\
\mathrm{~kg} \cdot \mathrm{m}^{-2} \\
\mathrm{~kg} \cdot \mathrm{m}^{-2} \\
\mathrm{~kg} \cdot \mathrm{m}^{-2} \\
\mathrm{~kg} \cdot \mathrm{m}^{-2}\end{array}$ & Pesticide emission & NA \\
\hline
\end{tabular}


Table A2. Cont.

\begin{tabular}{|c|c|c|c|c|c|c|c|}
\hline$\#$ & Reference & Data Source & Practice & Input & Unit & Output & Unit \\
\hline 70 & {$[88]$} & $\begin{array}{l}\text { Primary data (survey } \\
\text { and interview) } \\
\text { Secondary data } \\
\text { (Databases and } \\
\text { previous studies) }\end{array}$ & NA & $\begin{array}{l}\text { Cut flowers } \\
\text { Carnation support net } \\
\text { Plastic cover material } \\
\text { Putty for sun protection } \\
\text { Water for the putty used for sun } \\
\text { protection } \\
\text { Transporting of cut flowers to } \\
\text { Athens (2 times per week) } \\
\text { Electricity consumption for } \\
\text { refrigeration of the cut flowers, } \\
\text { water pumping } \\
\text { Fertilizers } \\
\text { Water for plant protection } \\
\text { Fungicides, Pesticides } \\
\text { Soil disinfection (once every three } \\
\text { years) } \\
\text { Water for soil disinfection, plant } \\
\text { watering ( } 3 \text { times per week) } \\
\text { Humidification }\end{array}$ & $\begin{array}{l}\text { Stems/year } \\
\mathrm{kg} \text { /year } \\
\mathrm{kg} \text { /year } \\
\mathrm{kg} \text { /year } \\
\mathrm{m}^{3} \text { /year } \\
\mathrm{km} / \text { year } \\
\mathrm{kWh} / \text { year } \\
\mathrm{kg} \text { N/year, } \mathrm{kg} \\
\mathrm{P} / \text { year, } \mathrm{kg} \\
\mathrm{K} / \text { year } \\
\mathrm{m}^{3} \text { /year } \\
\mathrm{kg} \text { /year } \\
\mathrm{kg} \text { /year } \\
\mathrm{m}^{3} \text { /year }\end{array}$ & $\mathrm{N}_{2} \mathrm{O}, \mathrm{NO}_{\mathrm{x}}$, and ammonia & NA \\
\hline 71 & [28] & $\begin{array}{l}\text { Secondary data } \\
\text { (databases and previous } \\
\text { studies) }\end{array}$ & Pesticide application & $\begin{array}{l}\text { Abamectine } \\
\text { Azoxystrobin } \\
\text { Benomyl } \\
\text { Bromopropylate } \\
\text { Captan } \\
\text { Cyromazine } \\
\text { Deltametrin } \\
\text { Fenarimol } \\
\text { Iprodione } \\
\text { Kresoxim-metil } \\
\text { Mancozeb } \\
\text { Pimetrozine }\end{array}$ & $\begin{array}{l}\mathrm{kgai} \cdot \mathrm{FU}^{-1} \\
\mathrm{kgai} \cdot \mathrm{FU}^{-1} \\
\mathrm{kgai} \cdot \mathrm{FU}^{-1} \\
\mathrm{kgai} \cdot \mathrm{FU}^{-1} \\
\mathrm{kgai} \cdot \mathrm{FU}^{-1} \\
\mathrm{kgai} \cdot \mathrm{FU}^{-1} \\
\mathrm{kgai} \cdot \mathrm{FU}^{-1} \\
\mathrm{kgai} \cdot \mathrm{FU}^{-1} \\
\mathrm{kgai} \cdot \mathrm{FU}^{-1} \\
\mathrm{kgai} \cdot \mathrm{FU}^{-1} \\
\text { kgai} \cdot \mathrm{FU}^{-1} \\
\mathrm{kgai} \cdot \mathrm{FU}^{-1}\end{array}$ & $\begin{array}{l}\text { Pesticide emission to air, soil, and } \\
\text { water }\end{array}$ & NA \\
\hline
\end{tabular}


Table A2. Cont.

\begin{tabular}{|c|c|c|c|c|c|c|c|}
\hline$\#$ & Reference & Data Source & Practice & Input & Unit & Output & Unit \\
\hline 72 & [12] & $\begin{array}{l}\text { Secondary data } \\
\text { (databases) }\end{array}$ & $\begin{array}{l}\text { Fertilizer application } \\
\text { Seeds use } \\
\text { Plant protection production } \\
\text { application } \\
\text { Agriculture activity }\end{array}$ & N, P, K fertilizer & NA & $\begin{array}{l}\mathrm{NH}_{3} \\
\mathrm{NO}_{x} \\
\mathrm{~N}_{2} \mathrm{O} \\
\mathrm{NO}_{3} \\
\mathrm{PO}_{4}{ }^{-3} \\
\mathrm{P} \\
\mathrm{HM}^{\mathrm{a}} \\
\text { Heavy metal } \\
\text { Active ingredients } \\
\mathrm{CO}_{2} \\
\mathrm{NMVOC} \\
\mathrm{PM}\end{array}$ & NA \\
\hline 73 & [102] & $\begin{array}{l}\text { Secondary data } \\
\text { (databases) }\end{array}$ & NA & $\begin{array}{l}\text { Pesticide } \\
\text { N Fertilizer } \\
\text { P Fertilizer }\end{array}$ & NA & $\begin{array}{l}\mathrm{N}_{2} \mathrm{O} \text {, air emission } \\
\mathrm{P} \text {, water } \\
\text { Emission } \\
\mathrm{NO}^{-3} \text {, water } \\
\text { Emission } \\
\text { Pesticides, } \\
\text { water } \\
\text { emission }\end{array}$ & NA \\
\hline
\end{tabular}




\section{References}

1. Kucukvar, M.; Ismaen, R.; Onat, N.C.; Al-Hajri, A.; Al-Yafay, H.; Al-Darwish, A. Exploring the Social, Economic and Environmental Footprint of Food Consumption: A Supply Chain-Linked Sustainability Assessment. In Proceedings of the 2019 IEEE 6th International Conference on Industrial Engineering and Applications (ICIEA), Tokyo, Japan, 12-15 April 2019; IEEE: Piscataway, NJ, USA; pp. 733-742.

2. Institute of Medicine and National Research Council. 2 Overview of the U.S. Food System. In A Framework for Assessing Effects of the Food System; The National Academies Press: Washington, DC, USA, 2015. Available online: https://www.nap.edu/read/1884 6/chapter / 5 (accessed on 28 September 2021). [CrossRef]

3. Tilman, D.; Balzer, C.; Hill, J.; Befort, B.L. Global Food Demand and the Sustainable Intensification of Agriculture. Proc. Natl Acad. Sci. USA 2011, 108, 20260-20264. [CrossRef] [PubMed]

4. Brandt, K.; Barrangou, R. Applications of CRISPR Technologies Across the Food Supply Chain. Annu. Rev. Food Sci. Technol. 2019, 10, 133-150. [CrossRef] [PubMed]

5. FAO; WFP; IFAD. The State of Food Insecurity in the World 2012. Economic Growth Is Necessary but Not Sufficient to Accelerate Reduction of Hunger and Malnutrition; FAO: Rome, Italy, 2012; p. 5. Available online: https:/ /www.fao.org/3/i3027e/i3027e00.pdf (accessed on 28 September 2021).

6. Sources of Greenhouse Gas Emissions. US EPA. Available online: https://www.epa.gov/ghgemissions/sources-greenhouse-gasemissions (accessed on 28 July 2021).

7. US EPA. Overview of Greenhouse Gases. Available online: https:/ /www.epa.gov/ghgemissions/overview-greenhouse-gases (accessed on 28 July 2021).

8. Cellura, M.; Longo, S.; Mistretta, M. Life Cycle Assessment (LCA) of Protected Crops: An Italian Case Study. J. Clean. Prod. 2012, 28, 56-62. [CrossRef]

9. Von Blottnitz, H.; Curran, M.A. A Review of Assessments Conducted on Bio-Ethanol as a Transportation Fuel from a Net Energy, Greenhouse Gas, and Environmental Life Cycle Perspective. J. Clean. Prod. 2007, 15, 607-619. [CrossRef]

10. Lee, K.-M.; Inaba, A. Life Cycle Assessment Best Practices of ISO 14040 Series; Center for Ecodesign and LCA (CEL), Ajou University: Suwon, Korea, 2004; p. 96.

11. Buyle, M.; Braet, J.; Audenaert, A. Life Cycle Assessment in the Construction Sector: A Review. Renew. Sustain. Energy Rev. 2013, 26, 379-388. [CrossRef]

12. Corrado, S.; Castellani, V.; Zampori, L.; Sala, S. Systematic Analysis of Secondary Life Cycle Inventories When Modelling Agricultural Production: A Case Study for Arable Crops. J. Clean. Prod. 2018, 172, 3990-4000. [CrossRef] [PubMed]

13. Atenstaedt, R. Word Cloud Analysis of the BJGP. Br. J. Gen. Pract. 2012, 62, 148. [CrossRef] [PubMed]

14. Heimerl, F.; Lohmann, S.; Lange, S.; Ertl, T. Word Cloud Explorer: Text Analytics Based on Word Clouds. In Proceedings of the 2014 47th Hawaii International Conference on System Sciences, Waikoloa, HI, USA, 6-9 January 2014; pp. $1833-1842$.

15. DePaolo, C.A.; Wilkinson, K. Get Your Head into the Clouds: Using Word Clouds for Analyzing Qualitative Assessment Data. TechTrends 2014, 58, 38-44. [CrossRef]

16. Present Your Data in A Doughnut Chart. Available online: https://support.microsoft.com/en-us/office/present-your-data-in-adoughnut-chart-0ac0efde-34e2-4dc6-9b7f-ac93d1783353 (accessed on 4 October 2021).

17. What Is A Pie Chart and When to Use It. Available online: https://www.storytellingwithdata.com/blog/2020/5/14/what-is-apie-chart (accessed on 4 October 2021).

18. Wu, J. Improving the Writing of Research Papers: IMRAD and Beyond. Landsc. Ecol. 2011, 26, 1345-1349. [CrossRef]

19. Vyas, V.d.H.A. Tantraguna-The Ancient Criteria for Scientific Writing. Ayu 2016, 37, 158-162. [CrossRef] [PubMed]

20. Nair, P.K.R.; Nair, V.D. Organization of a Research Paper: The IMRAD Format. In Scientific Writing and Communication in Agriculture and Natural Resources; Nair, P.K.R., Nair, V.D., Eds.; Springer International Publishing: Cham, Switzerland, 2014; pp. 13-25.

21. Hertwich, E.G.; Pease, W.S. ISO 14042 Restricts Use and Development of Impact Assessment. Int. J. Life Cycle Assess. 1998, 3, 180-181. [CrossRef]

22. Pryshlakivsky, J.; Searcy, C. Fifteen Years of ISO 14040: A Review. J. Clean. Prod. 2013, 57, 115-123. [CrossRef]

23. Fava, J.; Baer, S.; Cooper, J. Increasing Demands for Life Cycle Assessments in North America. J. Ind. Ecol. 2009, 13, 491-494. [CrossRef]

24. Huijbregts, M. Uncertainty and Variability in Environmental Life-Cycle Assessment. Int. J. Life Cycle Assess. 2002, 7, 173. [CrossRef]

25. Bhatia, P.; Cummis, C.; Draucker, L.; Rich, D.; Lahd, H.; Brown (WBCSD), A. Greenhouse Gas Protocol Product Life Cycle Accounting and Reporting Standard; World Resources Institute (WRI) and World Business Council for Sustainable Development (WBCSD): Washington, DC, USA, 2011.

26. Heijungs, R.; Guinée, J.B. (Eds.) Environmental Life Cycle Assessment of Products; Centre of Environmental Science: Leiden, The Netherlands, 1992.

27. Lueddeckens, S.; Saling, P.; Guenther, E. Temporal Issues in Life Cycle Assessment-A Systematic Review. Int. J. Life Cycle Assess. 2020, 25, 1385-1401. [CrossRef] 
28. Antón, A.; Castells, F.; Montero, J.I.; Huijbregts, M. Comparison of Toxicological Impacts of Integrated and Chemical Pest Management in Mediterranean Greenhouses. Chemosphere 2004, 54, 1225-1235. [CrossRef] [PubMed]

29. Huijbregts, M.A.J.; Struijs, J.; Goedkoop, M.; Heijungs, R.; Jan Hendriks, A.; van de Meent, D. Human Population Intake Fractions and Environmental Fate Factors of Toxic Pollutants in Life Cycle Impact Assessment. Chemosphere 2005, 61, 1495-1504. [CrossRef] [PubMed]

30. Jolliet, O.; Margni, M.; Charles, R.; Humbert, S.; Payet, J.; Rebitzer, G.; Rosenbaum, R. IMPACT 2002+: A New Life Cycle Impact Assessment Methodology. Int. J. Life Cycle Assess. 2003, 8, 324. [CrossRef]

31. de García, S.O.; García-Encina, P.A.; Irusta-Mata, R. The Potential Ecotoxicological Impact of Pharmaceutical and Personal Care Products on Humans and Freshwater, Based on USEtox ${ }^{\mathrm{TM}}$ Characterization Factors. A Spanish Case Study of Toxicity Impact Scores. Sci. Total Environ. 2017, 609, 429-445. [CrossRef]

32. Van Der Werf, H.M.G. Life Cycle Analysis of Field Production of Fibre Hemp, the Effect of Production Practices on Environmental Impacts. Euphytica 2004, 140, 13-23. [CrossRef]

33. Charles, R.; Jolliet, O.; Gaillard, G.; Pellet, D. Environmental Analysis of Intensity Level in Wheat Crop Production Using Life Cycle Assessment. Agric. Ecosyst. Environ. 2006, 113, 216-225. [CrossRef]

34. Brentrup, F.; Küsters, J.; Lammel, J.; Barraclough, P.; Kuhlmann, H. Environmental Impact Assessment of Agricultural Production Systems Using the Life Cycle Assessment (LCA) Methodology II. The Application to N Fertilizer Use in Winter Wheat Production Systems. Eur. J. Agron. 2004, 20, 265-279. [CrossRef]

35. Nesheim, M.C.; Oria, M.; Yih, P.T.; Committee on a Framework for Assessing the Health, Environmental, and Social Effects of the Food System; Food and Nutrition Board; Board on Agriculture and Natural Resources; Institute of Medicine; National Research Council. Environmental Effects of the U.S. Food System; National Academies Press: Washington, DC, USA, 2015.

36. Gathorne-Hardy, A. A life cycle assessment (LCA) of greenhouse gas emissions from SRI and flooded rice production in SE India. Taiwan Water Conserv. J. 2013, 61, 111-125.

37. Hanieh, A.A.; Hasan, A.; Assi, M. Date Palm Trees Supply Chain and Sustainable Model. J. Clean. Prod. 2020, $258,120951$. [CrossRef]

38. Fusi, A.; Castellani, V.; Bacenetti, J.; Cocetta, G.; Fiala, M.; Guidetti, R. The Environmental Impact of the Production of Fresh Cut Salad: A Case Study in Italy. Int. J. Life Cycle Assess. 2016, 21, 162-175. [CrossRef]

39. Tasca, A.L.; Nessi, S.; Rigamonti, L. Environmental Sustainability of Agri-Food Supply Chains: An LCA Comparison between Two Alternative Forms of Production and Distribution of Endive in Northern Italy. J. Clean. Prod. 2017, 140, 725-741. [CrossRef]

40. Reckenholz-Tänikon, A. Towards a Sustainable Management of the Food Chain. In Proceedings of the Research Station ART 6th International Conference on Life Cycle Assessment in the Agri-Food Sector, Zurich, Switzerland, 12-14 November 2008.

41. O'Neill, B.C.; Kriegler, E.; Ebi, K.L.; Kemp-Benedict, E.; Riahi, K.; Rothman, D.S.; van Ruijven, B.J.; van Vuuren, D.P.; Birkmann, J.; Kok, K. The Roads Ahead: Narratives for Shared Socioeconomic Pathways Describing World Futures in the 21st Century. Glob. Environ. Chang. 2017, 42, 169-180. [CrossRef]

42. Abeliotis, K.; Detsis, V.; Pappia, C. Life Cycle Assessment of Bean Production in the Prespa National Park, Greece. J. Clean. Prod. 2013, 41, 89-96. [CrossRef]

43. Beccali, M.; Cellura, M.; Iudicello, M.; Mistretta, M. Resource Consumption and Environmental Impacts of the Agrofood Sector: Life Cycle Assessment of Italian Citrus-Based Products. Environ. Manag. 2009, 43, 707-724. [CrossRef] [PubMed]

44. Blengini, G.A.; Busto, M. The Life Cycle of Rice: LCA of Alternative Agri-Food Chain Management Systems in Vercelli (Italy). J. Environ. Manag. 2009, 90, 1512-1522. [CrossRef] [PubMed]

45. Garofalo, P.; D’Andrea, L.; Tomaiuolo, M.; Venezia, A.; Castrignanò, A. Environmental Sustainability of Agri-Food Supply Chains in Italy: The Case of the Whole-Peeled Tomato Production under Life Cycle Assessment Methodology. J. Food Eng. 2017, 200, 1-12. [CrossRef]

46. Radzyminska, M.; Garbowska, B.; Jakubowska, D. Health Quality and Nutritional Value of Rye Bread Produced on a Small and Large Scale in Poland. Ital. J. Food Sci. 2013, 25, 126.

47. Ingwersen, W.W. Life Cycle Assessment of Fresh Pineapple from Costa Rica. J. Clean. Prod. 2012, 35, 152-163. [CrossRef]

48. Khoshnevisan, B.; Rafiee, S.; Omid, M.; Mousazadeh, H.; Clark, S. Environmental Impact Assessment of Tomato and Cucumber Cultivation in Greenhouses Using Life Cycle Assessment and Adaptive Neuro-Fuzzy Inference System. J. Clean. Prod. 2014, 73, 183-192. [CrossRef]

49. Bojacá, C.R.; Wyckhuys, K.A.; Schrevens, E. Life Cycle Assessment of Colombian Greenhouse Tomato Production Based on Farmer-Level Survey Data. J. Clean. Prod. 2014, 69, 26-33. [CrossRef]

50. Beccali, M.; Cellura, M.; Iudicello, M.; Mistretta, M. Life Cycle Assessment of Italian Citrus-Based Products. Sensitivity Analysis and Improvement Scenarios. J. Environ. Manag. 2010, 91, 1415-1428. [CrossRef]

51. Contreras, A.M.; Rosa, E.; Pérez, M.; Van Langenhove, H.; Dewulf, J. Comparative Life Cycle Assessment of Four Alternatives for Using By-Products of Cane Sugar Production. J. Clean. Prod. 2009, 17, 772-779. [CrossRef]

52. Hospido, A.; Milà i Canals, L.; McLaren, S.; Truninger, M.; Edwards-Jones, G.; Clift, R. The Role of Seasonality in Lettuce Consumption: A Case Study of Environmental and Social Aspects. Int. J. Life Cycle Assess. 2009, 14, 381-391. [CrossRef]

53. Roy, P.; Nei, D.; Okadome, H.; Nakamura, N.; Orikasa, T.; Shiina, T. Life Cycle Inventory Analysis of Fresh Tomato Distribution Systems in Japan Considering the Quality Aspect. J. Food Eng. 2008, 86, 225-233. [CrossRef] 
54. Broek, R.; Treffers, D.-J.; Meeusen, M.; Wijk, A.; Nieuwlaar, E.; Turkenburg, W. Green Energy or Organic Food? A Life-Cycle Assessment Comparing Two Uses of Set-Aside Land. J. Ind. Ecol. 2001, 5, 65-87. [CrossRef]

55. Masuda, K. Measuring Eco-Efficiency of Wheat Production in Japan: A Combined Application of Life Cycle Assessment and Data Envelopment Analysis. J. Clean. Prod. 2016, 126, 373-381. [CrossRef]

56. Andersson, K.; Ohlsson, T.; Olsson, P. Screening Life Cycle Assessment (LCA) of Tomato Ketchup: A Case Study. J. Clean. Prod. 1998, 6, 277-288. [CrossRef]

57. Choo, Y.M.; Muhamad, H.; Hashim, Z.; Subramaniam, V.; Puah, C.W.; Tan, Y. Determination of GHG Contributions by Subsystems in the Oil Palm Supply Chain Using the LCA Approach. Int. J. Life Cycle Assess. 2011, 16, 669-681. [CrossRef]

58. Romero-Gámez, M.; Audsley, E.; Suárez-Rey, E.M. Life Cycle Assessment of Cultivating Lettuce and Escarole in Spain. J. Clean. Prod. 2014, 73, 193-203. [CrossRef]

59. Girgenti, V.; Peano, C.; Bounous, M.; Baudino, C. A Life Cycle Assessment of Non-Renewable Energy Use and Greenhouse Gas Emissions Associated with Blueberry and Raspberry Production in Northern Italy. Sci. Total Environ. 2013, 458, 414-418. [CrossRef] [PubMed]

60. Breiling, M.; Hoshino, T.; Matsuhashi, R. Contributions of Rice Production to Japanese Greenhouse Gas Emissions Applying Life Cycle Assessment as a Methodology; The University of Tokyo: Tokyo, Japan, 1999; p. 32.

61. Kulak, M.; Graves, A.; Chatterton, J. Reducing Greenhouse Gas Emissions with Urban Agriculture: A Life Cycle Assessment Perspective. Landsc. Urban Plan. 2013, 111, 68-78. [CrossRef]

62. Vinyes, E.; Asin, L.; Alegre, S.; Muñoz, P.; Boschmonart, J.; Gasol, C.M. Life Cycle Assessment of Apple and Peach Production, Distribution and Consumption in Mediterranean Fruit Sector. J. Clean. Prod. 2017, 149, 313-320. [CrossRef]

63. Tabatabaie, S.M.H.; Murthy, G.S. Cradle to Farm Gate Life Cycle Assessment of Strawberry Production in the United States. J. Clean. Prod. 2016, 127, 548-554. [CrossRef]

64. Li, X.; Mupondwa, E.; Panigrahi, S.; Tabil, L.; Adapa, P. Life Cycle Assessment of Densified Wheat Straw Pellets in the Canadian Prairies. Int. J. Life Cycle Assess. 2012, 17, 420-431. [CrossRef]

65. Sahle, A.; Potting, J. Environmental Life Cycle Assessment of Ethiopian Rose Cultivation. Sci. Total Environ. 2013, 443, 163-172. [CrossRef]

66. Payen, S.; Basset-Mens, C.; Perret, S. LCA of Local and Imported Tomato: An Energy and Water Trade-Off. J. Clean. Prod. 2015, 87, 139-148. [CrossRef]

67. Roer, A.-G.; Korsaeth, A.; Henriksen, T.M.; Michelsen, O.; Strømman, A.H. The Influence of System Boundaries on Life Cycle Assessment of Grain Production in Central Southeast Norway. Agric. Syst. 2012, 111, 75-84. [CrossRef]

68. Matsuura, M.I.S.F.; Dias, F.R.T.D.; Picoli, J.F.; Lucas, K.R.G.; de Castro, C.; Hirakuri, M.H. Life-Cycle Assessment of the SoybeanSunflower Production System in the Brazilian Cerrado. Int. J. Life Cycle Assess. 2017, 22, 492-501. [CrossRef]

69. Nemecek, T.; Von Richthofen, J.-S.; Dubois, G.; Casta, P.; Charles, R.; Pahl, H. Environmental Impacts of Introducing Grain Legumes into European Crop Rotations. Eur. J. Agron. 2008, 28, 380-393. [CrossRef]

70. Martínez-Blanco, J.; Muñoz, P.; Antón, A.; Rieradevall, J. Assessment of Tomato Mediterranean Production in Open-Field and Standard Multi-Tunnel Greenhouse, with Compost or Mineral Fertilizers, from an Agricultural and Environmental Standpoint. J. Clean. Prod. 2011, 19, 985-997. [CrossRef]

71. Meisterling, K.; Samaras, C.; Schweizer, V. Decisions to Reduce Greenhouse Gases from Agriculture and Product Transport: LCA Case Study of Organic and Conventional Wheat. J. Clean. Prod. 2009, 17, 222-230. [CrossRef]

72. Canals, L.M.I.; Muñoz, I.; Hospido, A.; Plassmann, K.; McLaren, S.; Edwards-Jones, G.; Hounsome, B. Life cycle assessment (LCA) of domestic vs. imported vegetables. In Case Studies on Broccoli, Salad Crops and Green Beans; In RELU Project REW-224-25-0044; Centre for Environmental Strategy, University of Surrey: Guildford, UK, 2008.

73. Aguilera, E.; Guzmán, G.; Alonso, A. Greenhouse Gas Emissions from Conventional and Organic Cropping Systems in Spain. II. Fruit Tree Orchards. Agron. Sustain. Dev. 2015, 35, 725-737. [CrossRef]

74. Dwivedi, P.; Spreen, T.; Goodrich-Schneider, R. Global Warming Impact of Florida's Not-From-Concentrate (NFC) Orange Juice. Agric. Syst. 2012, 108, 104-111. [CrossRef]

75. Girgenti, V.; Peano, C.; Baudino, C.; Tecco, N. From “Farm to Fork" Strawberry System: Current Realities and Potential Innovative Scenarios from Life Cycle Assessment of Non-Renewable Energy Use and Green House Gas Emissions. Sci. Total Environ. 2014, 473, 48-53. [CrossRef] [PubMed]

76. Lazzerini, G.; Lucchetti, S.; Nicese, F.P. Green House Gases(GHG) Emissions from the Ornamental Plant Nursery Industry: A Life Cycle Assessment(LCA) Approach in a Nursery District in Central Italy. J. Clean. Prod. 2016, 112, 4022-4030. [CrossRef]

77. Mohseni, P.; Borghei, A.M.; Khanali, M. Coupled Life Cycle Assessment and Data Envelopment Analysis for Mitigation of Environmental Impacts and Enhancement of Energy Efficiency in Grape Production. J. Clean. Prod. 2018, 197, 937-947. [CrossRef]

78. Milà i Canals, L.; Burnip, G.M.; Cowell, S.J. Evaluation of the Environmental Impacts of Apple Production Using Life Cycle Assessment (LCA): Case Study in New Zealand. Agric. Ecosyst. Environ. 2006, 114, 226-238. [CrossRef]

79. Steenwerth, K.L.; Strong, E.B.; Greenhut, R.F.; Williams, L.; Kendall, A. Life Cycle Greenhouse Gas, Energy, and Water Assessment of Wine Grape Production in California. Int. J. Life Cycle Assess. 2015, 20, 1243-1253. [CrossRef]

80. Langevin, B.; Basset-Mens, C.; Lardon, L. Inclusion of the Variability of Diffuse Pollutions in LCA for Agriculture: The Case of Slurry Application Techniques. J. Clean. Prod. 2010, 18, 747-755. [CrossRef] 
81. Brentrup, F.; Küsters, J.; Kuhlmann, H.; Lammel, J. Application of the Life Cycle Assessment Methodology to Agricultural Production: An Example of Sugar Beet Production with Different Forms of Nitrogen Fertilisers. Eur. J. Agron. 2001, 14, 221-233. [CrossRef]

82. Nemecek, T.; Erzinger, S. Modelling Representative Life Cycle Inventories for Swiss Arable Crops (9 Pp). Int. J. Life Cycle Assess. 2005, 10, 68-76. [CrossRef]

83. Krzyżaniak, M.; Stolarski, M.J.; Warmiński, K. Life Cycle Assessment of Poplar Production: Environmental Impact of Different Soil Enrichment Methods. J. Clean. Prod. 2019, 206, 785-796. [CrossRef]

84. Lebailly, F.; Levasseur, A.; Samson, R.; Deschênes, L. Development of a Dynamic LCA Approach for the Freshwater Ecotoxicity Impact of Metals and Application to a Case Study Regarding Zinc Fertilization. Int. J. Life Cycle Assess. 2014, 19, 1745-1754. [CrossRef]

85. Mouron, P.; Nemecek, T.; Scholz, R.W.; Weber, O. Management Influence on Environmental Impacts in an Apple Production System on Swiss Fruit Farms: Combining Life Cycle Assessment with Statistical Risk Assessment. Agric. Ecosyst. Environ. 2006, 114, 311-322. [CrossRef]

86. Khanali, M.; Shahvarooghi Farahani, S.; Shojaei, H.; Elhami, B. Life Cycle Environmental Impacts of Saffron Production in Iran. Environ. Sci. Pollut. Res. Int. 2017, 24, 4812-4821. [CrossRef] [PubMed]

87. Andrianandraina; Ventura, A.; Senga Kiessé, T.; Cazacliu, B.; Idir, R.; Werf, H.M.G. Sensitivity Analysis of Environmental Process Modeling in a Life Cycle Context: A Case Study of Hemp Crop Production. J. Ind. Ecol. 2015, 19, 978-993. [CrossRef]

88. Abeliotis, K.; Barla, S.-A.; Detsis, V.; Malindretos, G. Life Cycle Assessment of Carnation Production in Greece. J. Clean. Prod. 2016, 112, 32-38. [CrossRef]

89. Deytieux, V.; Nemecek, T.; Freiermuth Knuchel, R.; Gaillard, G.; Munier-Jolain, N.M. Is Integrated Weed Management Efficient for Reducing Environmental Impacts of Cropping Systems? A Case Study Based on Life Cycle Assessment. Eur. J. Agron. 2012, 36, 55-65. [CrossRef]

90. Peña, N.; Knudsen, M.T.; Fantke, P.; Antón, A.; Hermansen, J.E. Freshwater Ecotoxicity Assessment of Pesticide Use in Crop Production: Testing the Influence of Modeling Choices. J. Clean. Prod. 2019, 209, 1332-1341. [CrossRef]

91. Xue, X.; Hawkins, T.R.; Ingwersen, W.W.; Smith, R.L. Demonstrating an Approach for Including Pesticide Use in Life-Cycle Assessment: Estimating Human and Ecosystem Toxicity of Pesticide Use in Midwest Corn Farming. Int. J. Life Cycle Assess. 2015, 20, 1117-1126. [CrossRef]

92. Juraske, R.; Sanjuán, N. Life Cycle Toxicity Assessment of Pesticides Used in Integrated and Organic Production of Oranges in the Comunidad Valenciana, Spain. Chemosphere 2011, 82, 956-962. [CrossRef]

93. Khoshnevisan, B.; Bolandnazar, E.; Shamshirband, S.; Shariati, H.M.; Anuar, N.B.; Mat Kiah, M.L. Decreasing Environmental Impacts of Cropping Systems Using Life Cycle Assessment (LCA) and Multi-Objective Genetic Algorithm. J. Clean. Prod. 2015, 86, 67-77. [CrossRef]

94. Schmidt Rivera, X.C.; Bacenetti, J.; Fusi, A.; Niero, M. The Influence of Fertiliser and Pesticide Emissions Model on Life Cycle Assessment of Agricultural Products: The Case of Danish and Italian Barley. Sci. Total Environ. 2017, 592, 745-757. [CrossRef] [PubMed]

95. Brodt, S.; Kramer, K.J.; Kendall, A.; Feenstra, G. Comparing Environmental Impacts of Regional and National-Scale Food Supply Chains: A Case Study of Processed Tomatoes. Food Policy 2013, 42, 106-114. [CrossRef]

96. Theurl, M.C.; Haberl, H.; Erb, K.-H.; Lindenthal, T. Contrasted Greenhouse Gas Emissions from Local versus Long-Range Tomato Production. Agron. Sustain. Dev. 2014, 34, 593-602. [CrossRef]

97. Iriarte, A.; Rieradevall, J.; Gabarrell, X. Life Cycle Assessment of Sunflower and Rapeseed as Energy Crops under Chilean Conditions. J. Clean. Prod. 2010, 18, 336-345. [CrossRef]

98. Schmidt, J.H. Comparative Life Cycle Assessment of Rapeseed Oil and Palm Oil. Int. J. Life Cycle Assess. 2010, 15, 183-197. [CrossRef]

99. Noya, I.; González-García, S.; Bacenetti, J.; Arroja, L.; Moreira, M.T. Comparative Life Cycle Assessment of Three Representative Feed Cereals Production in the Po Valley (Italy). J. Clean. Prod. 2015, 99, 250-265. [CrossRef]

100. Nemecek, T.; Kägi, T. Life Cycle Inventories of Agricultural Production Systems. Final Rep. Ecoinvent 2007, 361. [CrossRef]

101. Antón, A.; Torrellas, M.; Núñez, M.; Sevigné, E.; Amores, M.J.; Muñoz, P.; Montero, J.I. Improvement of Agricultural Life Cycle Assessment Studies through Spatial Differentiation and New Impact Categories: Case Study on Greenhouse Tomato Production. Environ. Sci. Technol. 2014, 48, 9454-9462. [CrossRef] [PubMed]

102. Shrestha, P.; Karim, R.A.; Sieverding, H.L.; Archer, D.W.; Kumar, S.; Nleya, T.; Graham, C.J.; Stone, J.J. Life Cycle Assessment of Wheat Production and Wheat-Based Crop Rotations. J. Environ. Qual. 2020, 49, 1515-1529. [CrossRef] [PubMed]

103. Gentil, C.; Basset-Mens, C.; Manteaux, S.; Mottes, C.; Maillard, E.; Biard, Y.; Fantke, P. Coupling Pesticide Emission and Toxicity Characterization Models for LCA: Application to Open-Field Tomato Production in Martinique. J. Clean. Prod. 2020, 277, 124099. [CrossRef]

104. Brentrup, F.; Küsters, J.; Kuhlmann, H.; Lammel, J. Environmental Impact Assessment of Agricultural Production Systems Using the Life Cycle Assessment Methodology. Eur. J. Agron. 2004, 20, 247-264. [CrossRef]

105. Salomone, R.; Ioppolo, G. Environmental Impacts of Olive Oil Production: A Life Cycle Assessment Case Study in the Province of Messina (Sicily). J. Clean. Prod. 2012, 28, 88-100. [CrossRef]

106. Peano, C.; Baudino, C.; Tecco, N.; Girgenti, V. Green Marketing Tools for Fruit Growers Associated Groups: Application of the Life Cycle Assessment (LCA) for Strawberries and Berry Fruits Ecobranding in Northern Italy. J. Clean. Prod. 2015, $104,59-67$. [CrossRef] 